\title{
Density of states for the $\pi$-flux state with bipartite real random hopping only: A weak disorder approach
}

\author{
C. Mudry \\ Paul Scherrer Institute, CH-5232 Villigen PSI, Switzerland \\ and Yukawa Institute for Theoretical Physics, Kyoto University, Kyoto 606-8502, Japan \\ S. Ryu \\ Department of Applied Physics, University of Tokyo, 7-3-1 Hongo Bunkyo-ku, Tokyo 113-8656, Japan
}

A. Furusaki

Yukawa Institute for Theoretical Physics, Kyoto University, Kyoto 606-8502, Japan

(Received 30 July 2002; revised manuscript received 20 November 2002; published 28 February 2003)

\begin{abstract}
Gade [Nucl. Phys. B 398, 499 (1993)] has shown that the density of states for a particle hopping on a two-dimensional bipartite lattice in the presence of weak disorder and in the absence of time-reversal symmetry (chiral unitary universality class) is anomalous in the vicinity of the band center $\varepsilon=0$ whenever the disorder preserves the sublattice symmetry. More precisely, using a nonlinear $\sigma$ model that encodes the sublattice (chiral) symmetry and the absence of time-reversal symmetry she argues that the disorder average density of states diverges as $|\varepsilon|^{-1} \exp \left(-c|\ln \varepsilon|^{\kappa}\right)$ with $c$ some nonuniversal positive constant and $\kappa=1 / 2$ a universal exponent. Her analysis has been extended to the case when time-reversal symmetry is present (chiral orthogonal universality class) for which the same exponent $\kappa=1 / 2$ was predicted. Motrunich et al. [Phys. Rev. B $\mathbf{6 5}$, 064206 (2002)] have argued that the exponent $\kappa=1 / 2$ does not apply to the density of states in the chiral orthogonal universality class. They predict that $\kappa=2 / 3$ instead. We confirm the analysis of Motrunich et al. within a field theory for two flavors of Dirac fermions subjected to two types of weak uncorrelated random potentials: a purely imaginary vector potential and a complex valued mass potential. This model is the naive continuum limit of a model describing a particle hopping on a square lattice in the background of a $\pi$-flux phase and subjected to weak disorder that preserves the sublattice symmetry and time-reversal invariance. By commonly held universality arguments, this model is believed to belong to the chiral orthogonal universality class. Our calculation relies in an essential way on the existence of infinitely many local composite operators with negative anomalous scaling dimensions.
\end{abstract}

DOI: 10.1103/PhysRevB.67.064202

PACS number(s): 71.30.+h, 72.15.Rn, 64.60.Fr, 05.40.-a

\section{INTRODUCTION}

The effect of disorder on the density of states (DOS) in the problem of Anderson localization is known to be rather mild: In general, disorder smoothes the DOS. ${ }^{1,2}$ For example, the logarithmic Van Hove singularity in the DOS at the band center for a single-particle undergoing uniform nearestneighbor hopping on a square lattice is washed out by disorder in two dimensions (2D) ${ }^{3,4}$ Consequently, the signature of disorder-induced critical behavior in Anderson localization is usually not captured by the DOS, and one needs to resort to more complex probes such as conductance statistics, energy levels statistics, or multifractal spectra that are sensitive to a delocalization transition.

Exceptions to the rule that the DOS is smooth in Anderson localization can occur at the band edges or at the band center. ${ }^{2}$ The band center is very special whenever the disorder preserves "particle-hole" symmetry, i.e., a symmetry that guarantees that energy eigenvalues always occur in pairs of opposite signs for any given realization of the disorder. The particle-hole symmetry can take two different forms: (i) the sublattice symmetry ${ }^{5-10}$ (also called chiral symmetry) which is relevant in the context of Hamiltonians defined on bipartite lattices and is implemented by a unitary transformation (changing the sign of the wave functions on every site belonging to one sublattice but not on the other) and (ii) the Bogoliubov-de Gennes (BdG) symmetry ${ }^{11-13}$ which is relevant in the context of disordered superconductors and is implemented by an antiunitary transformation. ${ }^{14}$ The special role played by the band center when the disorder preserves particle-hole symmetry follows from the enhanced level repulsion felt by energy eigenvalues close to the band center as a result of their mirror images. Naively, one would expect that level repulsion results in a depletion of the DOS close to the band center. This expectation is in fact always realized in finite systems and is relevant to quantum dots or to level statistics in the core of vortices in a superconductor. ${ }^{15}$ However, it has been known since the work of Dyson ${ }^{5}$ on disordered one-dimensional chains that the DOS can, in spite of the enhanced level repulsion, diverge at the band center in the thermodynamic limit. The band center must then represent "some sort" of disorder-induced critical point characterized by a delocalization transition of "some kind." Indeed, imagine that there is no delocalization transition, i.e., that the localization length is finite at the band center. If so, the naive expectation of a depletion of the DOS as a result of enhanced level repulsion must apply in the thermodynamic limit as the system behaves in effect as a sampling of uncorrelated boxes 
of linear size of the order of the localization length for sufficiently long length scales.

Anderson localization with particle-hole symmetry thus represents a laboratory in which disorder-induced critical behavior can be studied both in 1D and 2D. In 1D a wealth of complementary approaches based on the nonlinear $\sigma$ model $(\mathrm{NLSM}){ }^{16,17}$ real-space renormalization group (RG) techniques, ${ }^{18}$ extensions of the Dorokhov-Mello-PereyraKumar (DMPK) equation, ${ }^{13,19,20}$ direct studies of critical zero modes, ${ }^{21,22}$ and others ${ }^{23-25}$ are available and have been used to provide a rather detailed description of the delocalization transition at the band center. In $2 \mathrm{D}$, the use of the NLSM was pioneered by Gade and Wegner in the context of Anderson localization with particle-hole symmetry implemented by a sublattice symmetry. ${ }^{8}$ They predicted that the disorder average DOS per unit volume and energy diverges upon approaching the band center $\varepsilon=0$ as

$$
|\varepsilon|^{-v} \exp \left(-c|\ln \varepsilon|^{\kappa}\right), \quad v=1, \quad \kappa=\frac{1}{2} .
$$

Here $c$ is a positive nonuniversal constant, whereas the exponents $v$ and $\kappa$ are universal. Moreover, neither $v$ nor $\kappa$ depends on whether time-reversal symmetry is broken or not. The NLSM approach of Gade and Wegner has been extended to the random $\pi$-flux phase ${ }^{26,27}$ and to the random flux model. ${ }^{28}$ It also has been refined to account for the expectation value of the staggered DOS (Ref. 29) and for the presence of Wess-Zumino-Witten (WZW) terms. ${ }^{27}$ In all cases, the DOS (1.1) was recovered. Particle-hole symmetry is also essential to understand the role played by disorder in a meanfield and non-self-consistent treatment of superconductivity. ${ }^{14}$ In 2D, the disorder average DOS was predicted $^{11,12}$ to diverge as

$$
|\ln \varepsilon|^{\kappa}
$$

when spin-rotation symmetry is broken. The universal exponent $\kappa=1 / 2$ in the presence of time-reversal symmetry and $\kappa=1$ in the absence of time-reversal symmetry.

Motrunich et al. in Ref. 30 have proposed that the exponent $\kappa$ is not given by $1 / 2$ as suggested by various fieldtheoretical calculations leading to Eq. (1.1) (see Refs. 8 and 26-29) but by the larger exponent

$$
\kappa=\frac{2}{3} .
$$

The method by which Motrunich et al. reach their conclusion in Ref. 30 is based on (i) a real-space RG analysis that is intrinsically a strong-disorder technique, ${ }^{31}$ (ii) a combination of exact variational bounds for strong random vector potential disorder with the computation of the DOS in the weakdisorder limit by Ludwig et al. in Ref. 9 and with the computation of the $\beta$ function for the variance of the random vector potential by Guruswamy et al. in Ref. 27, and (iii) a connection to the problem of monomer free energies in some problems of dimer covering. In this paper we undertake the task of understanding the discrepancy between Eqs. (1.1) and (1.3) for the Hatsugai-Wen-Kohmoto (HWK) model ${ }^{32}$ in the limit of weak disorder at microscopic length scales and within a purely field-theoretical approach. We shall show that the RG analysis for the average DOS in the HWK model performed in Ref. 27 that leads to Eq. (1.1) is not complete in that it fails to capture the broadness of the local density of states (LDOS) distribution. A consistent RG analysis for the DOS can, however, be constructed and yields Eq. (1.3) in the HWK model.

The HWK model is the naive continuum limit for a model of a particle undergoing nearest-neighbor hopping on a rectangular lattice in the background of $\pi$-flux phase. The microscopic disorder is introduced by allowing real-valued random fluctuations of the nearest-neighbor hopping amplitudes about the $\pi$-flux phase. The continuum limit of the clean spectrum is approximated by two flavors of Dirac fermions. The microscopic disorder is assumed to be sufficiently weak for the nodal structure of the clean energy spectrum to survive the perturbation by the disorder. It was shown in Ref. 32 that the disorder manifests itself by a purely imaginary random vector potential and by a random complex valued mass in the naive continuum limit. Furthermore, boundary conditions were chosen so as to guarantee the existence of a zero mode for any realization of the disorder and a numerical study of zero modes confirmed the theoretical expectation that zero modes are critical (multifractal). Numerical studies of the DOS for the HWK model are inconclusive due to severe limits on the achieved energy resolution. ${ }^{33,34}$ From a purely symmetry point of view the HWK model belongs to the chiral orthogonal universality class since time-reversal symmetry is preserved. If symmetries alone control the asymptotic behavior of the DOS in the vicinity of the band center, one would expect that the disorder average DOS is of the form given in Eq. (1.1). This is indeed what is found in Ref. 27, where it is shown that (i) the HWK model realizes a nearly conformal field theory and (ii) the DOS (1.1) follows from solving a Callan-Symanzik equation that depends on three coupling constants.

In this paper we shall build on the remarkable results (i) of Guruswamy et al. in Ref. 27 to identify an infinite but countable set of relevant local operators with negative anomalous scaling dimensions in the HWK model. The physical interpretation of these relevant operators is that they govern the dependence on energy $\varepsilon$ of all moments of the LDOS provided $\varepsilon$ is sufficiently close to the band center $\varepsilon$ $=0$. Their relevance reflects the fact that the probability distribution for the LDOS becomes very broadly distributed in the thermodynamic limit as its distribution is governed by the (multifractal) critical state at the band center. Correspondingly, we shall show that the disorder average DOS $\nu(\varepsilon)$ obeys a Callan-Symanzik equation that depends on infinitely many relevant coupling constants. Following an approximation developed by Carpentier and Le Doussal for the random phase $X Y$ model in $2 \mathrm{D},{ }^{35}$ we reduce the CallanSymanzik equation with infinitely many relevant coupling constants to the Callan-Symanzik equation of Guruswamy et al. with the caveat that the anomalous scaling dimension in (ii) that corresponds to the annealed average value of the dynamical exponent must be replaced by the anomalous scal- 
ing dimension that corresponds to the quenched average value of the dynamical exponent. In doing so we recover Eq. (1.3).

The physical origin of the infinite but countable family of operators with negative anomalous scaling dimensions can be traced to the multifractality of zero modes supported by the HWK model when the random complex mass is switched off. The explicit interplay between multifractality of zero modes and negative scaling dimensions in a conformal field theory (CFT) was studied in Refs. 9 and 36-41 for a single flavor of a Dirac fermion subjected to a random vector potential. It was shown in Refs. 38-40 that the multifractal spectrum is characterized by a freezing transition. The multifractal spectrum probes the scaling of the support of a normalized zero mode whose squared amplitude (height) is some prescribed value as a function of $L$ in a finite box of volume $L^{2}$. As the height is increased, the multifractal spectrum undergoes a freezing transition. Below the freezing transition, the support scales with the system size to some finite power. Above the freezing transition, the support does not scale anymore with the system size. A similar freezing transition occurs when the height is decreased towards a minimum value of the squared amplitude of zero modes. It was argued in Refs. 30 and 42 that the freezing transition in the multifractal spectrum mirrors itself in the DOS for a single Dirac fermion subjected to a random vector potential. The relation between the multifractal spectrum and the scaling of the DOS is embodied by the dynamical exponent that relates scaling with energy to scaling with system size. It is thus the dynamical exponent that undergoes a freezing transition. We will argue that the same mechanism applies, with suitable modifications, to the HWK model.

Although the emphasis is on Anderson localization, the models considered in this paper are also intimately connected to disordered Coulomb gases, random $X Y$, and other 2D disordered systems from classical statistical physics. ${ }^{30,35,36,38,41-44}$ Perhaps the most surprising connection between Anderson localization and a classical stochastic model is the one conjectured in Ref. 38 between the multifractal spectrum of zero modes and the freezing transition of directed polymers on a Cayley tree (see Refs. 45 and 46). Carpentier and Le Doussal have convincingly argued that a consistent RG analysis of the multifractal spectrum of zero modes reduces to solving the so-called KolmogorovPetrovsky-Piscounov (KPP) nonlinear diffusion equation. ${ }^{4}$ Their approach yields new insights into the universality of the multifractal spectrum in terms of the unique properties of the asymptotic solutions of the KPP equation. It also gives a RG justification for the conjecture in Ref. 38 by building on the fact that it is known from Ref. 45 that the transformation law of the generating function for the random partition function of directed polymers on a Cayley tree under an infinitesimal RG step is also governed by the KPP equation. Hereto we shall see that the Callan-Symanzik equation obeyed by the DOS in the HWK model is intimately related to the KPP equation.

Our paper is organized as follows. Lattice and continuum versions of the HWK Hamiltonians are defined in Sec. II where we also define the Motrunich-Damle-Huse (MDH) model. We choose a supersymmetric (SUSY) field theory to represent the DOS in Sec. III. After these preliminaries, we derive the DOS for the MDH model in Sec. IV. We compute the DOS for the HWK model in Sec. V. We conclude in Sec. VI. Some technical details are summarized in the Appendixes.

\section{DEFINITIONS}

\section{A. Lattice models}

We are concerned with the naive continuum limit of a subclass of lattice Hamiltonians belonging to the chiral orthogonal universality class. Consider some two-dimensional bipartite lattice-say, a lattice that is rectangular in the bulk. One sublattice is denoted $A$, the other $B$. There are $N_{A}$ sites on sublattice $A$ and $N_{B}$ sites on sublattice $B$. Without loss of generality, assume that $N_{A} \geqslant N_{B}$. The random Hamiltonian is then represented by

$$
\mathcal{H}=\left(\begin{array}{cc}
0 & T_{A B} \\
T_{B A} & 0
\end{array}\right),
$$

where $T_{A B}$ is a $N_{A} \times N_{B}$ real-valued matrix with the random matrix elements $\left(T_{A B}\right)_{i j}=t_{i j}$ if $i j$ are nearest-neighbor sites with $i \in A$ and $\left(T_{A B}\right)_{i j}=0$ otherwise, while the $N_{B} \times N_{A}$ matrix $T_{B A}$ is the transpose of $T_{A B}: T_{B A}=\left(T_{A B}\right)^{\mathrm{t}}$. Random Hamiltonians of the form (2.1) are generic representatives of the chiral orthogonal universality class. Wave functions in the $N$-dimensional Hilbert space $\left(N:=N_{A}+N_{B}\right)$ are represented by

$$
\Psi=\left(\begin{array}{c}
\Psi_{A} \\
\Psi_{B}
\end{array}\right) .
$$

By construction, $\mathcal{H}$ changes by a sign under the unitary transformation

$$
\Psi_{A} \rightarrow+\Psi_{A}, \quad \Psi_{B} \rightarrow-\Psi_{B} .
$$

Zero modes have the form

$$
\Psi=\left(\begin{array}{c}
\Psi_{A} \\
0
\end{array}\right)
$$

where

$$
0=T_{B A} \Psi_{A}=\left(T_{A B}\right)^{\mathrm{t}} \Psi_{A} .
$$

Zero modes are thus eigenstates of the unitary transformation (2.3). There are $N_{A}-N_{B}$ zero modes.

The HWK lattice model is given by the Hamiltonian $\mathcal{H}_{\text {HWK }}$ of the form (2.1) whereby it is assumed that (i) a $\pi$-flux phase threads every elementary plaquette $\square$ of the rectangular lattice and (ii) there are small random fluctuations $\delta t_{i j}$ of the nearest-neighbor hopping amplitude:

$$
t_{i j}=t_{i j}^{(0)}+\delta t_{i j}, \quad \prod_{i j \in \square} t_{i j}^{(0)}=-t^{4} .
$$

Here $t$ is the characteristic energy scale ( $\propto$ bandwidth) for the clean system. In Ref. 32, the multifractal spectrum of 
zero modes of $\mathcal{H}_{\mathrm{HWK}}$ was studied numerically and shown to agree within numerical error bars with a theoretical prediction made in Refs. 9 and 37-40. In subsequent papers the DOS was also studied numerically. ${ }^{33,34}$ Unfortunately, the lack of energy resolution was so severe that the observation of a diverging DOS could only be established in Ref. 34 through a tiny upturn of the DOS away from an algebraic decaying crossover regime.

Motrunich et al. in Ref. 30 proposed a different parametrization of the random fluctuations of the nearest-neighbor hopping amplitudes. Their lattice Hamiltonian $\mathcal{H}_{\mathrm{MDH}}$ is of the form (2.1) but with

$$
t_{i j}=e^{+\phi_{i}} t_{i j}^{(0)} e^{-\phi_{j}}, \quad \prod_{i j \in \square} t_{i j}^{(0)}=-t^{4},
$$

instead of Eq. (2.6). The $\phi$ 's are real-valued, white-noisecorrelated, Gaussian random variables of vanishing means. With the help of the $N_{A} \times N_{A}$ diagonal matrix

$$
U_{A}:=\left(\begin{array}{ccc}
e^{+\phi_{1}} & & \\
& \ddots & \\
& & e^{+\phi_{N_{A}}}
\end{array}\right)
$$

and of the $N_{B} \times N_{B}$ diagonal matrix

$$
U_{B}:=\left(\begin{array}{ccc}
e^{+\phi_{N_{A}+1}} & & \\
& \ddots & \\
& & e^{+\phi_{N_{A}+N_{B}}}
\end{array}\right) \text {, }
$$

the MDH random Hamiltonian becomes

$$
\mathcal{H}_{\mathrm{MDH}}=\left(\begin{array}{cc}
0 & U_{A} T_{A B}^{(0)}\left(U_{B}\right)^{-1} \\
\left(U_{B}\right)^{-1}\left(T_{A B}^{(0)}\right)^{\mathrm{t}} U_{A} & 0
\end{array}\right),
$$

where $T_{A B}^{(0)}$ is a $N_{A} \times N_{B}$ matrix made of $t_{i j}^{(0)}$. In this model, it is straightforward to construct explicitly zero modes. To each solution

$$
\Psi^{\prime}=\left(\begin{array}{c}
\Psi_{A}^{\prime} \\
0
\end{array}\right)
$$

of

$$
0=T_{B A}^{(0)} \Psi_{A}^{\prime}=\left(T_{A B}^{(0)}\right)^{t} \Psi_{A}^{\prime}
$$

[i.e., Eq. (2.5) without disorder], there corresponds a zero mode of $\mathcal{H}_{\mathrm{MDH}}$ given by

$$
\Psi=\left(\begin{array}{cc}
\left(U_{A}\right)^{-1} & 0 \\
0 & U_{B}
\end{array}\right) \Psi^{\prime}=\left(\begin{array}{c}
\left(U_{A}\right)^{-1} \Psi_{A}^{\prime} \\
0
\end{array}\right) .
$$

This explicit construction of zero modes will have a counterpart in the field theory later on and plays a very special role. The imaginary gauge chiral transformation (2.13) turns the Schrödinger equation at any finite $\varepsilon$ into

$$
\begin{gathered}
T_{A B}^{(0)} \Psi_{B}^{\prime}=\varepsilon\left(U_{A}\right)^{-2} \Psi_{A}^{\prime}, \\
\left(T_{A B}^{(0)}\right)^{t} \Psi_{A}^{\prime}=\varepsilon\left(U_{B}\right)^{+2} \Psi_{B}^{\prime} .
\end{gathered}
$$

\section{B. Naive continuum limit}

Common to the HWK and MDH lattice Hamiltonians in the clean limit is the property that the Fermi surface collapses to four Fermi points at ( $\pm \pi / 2, \pm \pi / 2)$ in the Brillouin zone at half filling. Correspondingly, the clean DOS has a V-shaped singularity at the band center. Linearization of the spectrum around those four Fermi points describes two copies of "relativistic" particles moving with the Fermi velocity $v_{f}:=2 \mathfrak{a} t, \mathfrak{a}$ being the lattice spacing. These are the two flavors of Dirac fermions. Unless specified, we will set $v_{f}$ to 1 .

The difference in the parametrization of the disorder in the HWK and MDH lattice Hamiltonians survives the continuum limit. For sufficiently weak disorder, the naive continuum limit for the HWK lattice Hamiltonian reads ${ }^{32}$

$$
H_{\mathrm{HWK}}=\left(\begin{array}{cc}
0 & D_{\mathrm{HWK}} \\
D_{\mathrm{HWK}}^{\dagger} & 0
\end{array}\right),
$$

with

$$
D_{\mathrm{HWK}}=i \sigma_{\mu} \partial_{\mu}+i \sigma_{\mu} A_{\mu}+i \sigma_{0} A_{0}+\sigma_{3} A_{3}
$$

and

$$
D_{\mathrm{HWK}}^{\dagger}=i \sigma_{\mu} \partial_{\mu}-i \sigma_{\mu} A_{\mu}-i \sigma_{0} A_{0}+\sigma_{3} A_{3} .
$$

Pauli matrices $\boldsymbol{\sigma} \equiv\left(\sigma_{1}, \sigma_{2}\right)$, together with the unit $2 \times 2$ matrix $\sigma_{0}$ and the third Pauli matrices $\sigma_{3}$ have been introduced. Summation over the repeated indices $(\mu=1,2)$ is assumed throughout this paper. This Hamiltonian is invariant under time reversal, i.e.,

$$
\mathcal{T}\left(H_{\mathrm{HWK}}\right)^{*} \mathcal{T}=H_{\mathrm{HWK}}, \quad \mathcal{T}:=\left(\begin{array}{cc}
\sigma_{1} & 0 \\
0 & -\sigma_{1}
\end{array}\right) .
$$

It also changes by a sign under charge conjugation

$$
\mathcal{C} H_{\mathrm{HWK}} \mathcal{C}=-H_{\mathrm{HWK}}, \quad \mathcal{C}:=\left(\begin{array}{cc}
\sigma_{0} & 0 \\
0 & -\sigma_{0}
\end{array}\right),
$$

which is nothing but the continuum counterpart of the transformation that implements the transformation $T_{A B} \rightarrow-T_{A B}$ in Eq. (2.3). The $\sigma$ 's encode the spinor grading of a single flavor of Dirac fermions. The (real-valued) disorder is represented by (i) the "purely imaginary" random vector potential $\mathbf{A}=\left(A_{1}, A_{2}\right) \in \mathbb{R}^{2}$, (ii) the "purely imaginary" random scalar potential (chemical potential) $A_{0} \in \mathbb{R}$, and (iii) the random mass potential $A_{3} \in \mathbb{R}$.

The terminology "purely imaginary" comes from the factor of $i$ multiplying $\mathbf{A}$ and $A_{0}$ in the operator $D_{\mathrm{HWK}}$; i.e., $\mathbf{A}$ and $A_{0}$ parametrize the anti-Hermitian contributions to $D_{\text {HWK }}$. The Hermitian contributions to $D_{\text {HwK }}$ are the kinetic energy $i \boldsymbol{\sigma} \cdot \boldsymbol{\partial}$ and the random mass contributions $\sigma_{3} A_{3}$. With a prejudice towards a field-theoretical approach, it is stan- 
dard practice to assume that $\mathbf{A}, A_{0}$, and $A_{3}$ are all independent white-noise-correlated Gaussian random variables of vanishing means. ${ }^{47}$ The implicit assumption made here is that the detailed shape of the disorder should not matter as long as it is weak and short-range correlated in space. So far the discussion is generic to the chiral orthogonal universality class. We now specialize to a subclass of the chiral orthogonal universality class by, following Hatsugai et al., making a further important simplification. Motivated by analytical continuation of a one-loop RG calculation performed in Ref. 43 for a single flavor of Dirac fermions subjected to "purely real" random scalar, vector, and mass potentials, ${ }^{48}$ we assume that, on the one hand, the two components of the vector potential have the same variance $g_{A}$ and, on the other hand, the random scalar potential and the random mass have the same variance $g_{M}$ (disorder averaging is denoted by an overbar):

$$
\overline{A_{\mu}(\boldsymbol{x}) A_{\nu}(\boldsymbol{y})}=\left\{\begin{array}{lc}
g_{A} \delta_{\mu \nu} \delta(\boldsymbol{x}-\boldsymbol{y}), & \mu, \nu=1,2, \\
g_{M} \delta_{\mu \nu} \delta(\boldsymbol{x}-\boldsymbol{y}), & \mu, \nu=0,3, \\
0, & \text { otherwise }
\end{array}\right.
$$

This completes the definition of the HWK model.

For sufficiently weak disorder, the naive continuum limit for the MDH Hamiltonian is given by Eq. (2.15a) without the purely imaginary random scalar potential and random mass. To see this, we demand a counterpart to Eqs. (2.10) and (2.13) in the continuum limit. First, we introduce the parametrization

$$
A_{\mu}=\widetilde{\partial}_{\mu} \Phi_{1}+\partial_{\mu} \Phi_{2} \quad\left(\widetilde{\partial}_{\mu}:=\epsilon_{\mu \nu} \partial_{\nu}, \quad \mu=1,2\right)
$$

of the random vector potential in terms of two independent scalar fields $\Phi_{1}$ and $\Phi_{2}$, where $\epsilon_{\mu \nu}$ is the antisymmetric tensor with $\epsilon_{12}=1$. Second, we observe that

$$
\begin{aligned}
& i \sigma_{\mu}\left(\partial_{\mu}+A_{\mu}\right)=e^{+i \sigma_{3} \Phi_{1}-\sigma_{0} \Phi_{2}} i \sigma_{\mu} \partial_{\mu} e^{-i \sigma_{3} \Phi_{1}+\sigma_{0} \Phi_{2}}, \\
& i \sigma_{\mu}\left(\partial_{\mu}-A_{\mu}\right)=e^{-i \sigma_{3} \Phi_{1}+\sigma_{0} \Phi_{2}} i \sigma_{\mu} \partial_{\mu} e^{+i \sigma_{3} \Phi_{1}-\sigma_{0} \Phi_{2}} .
\end{aligned}
$$

We thus identify Eqs. (2.8) and (2.9) with

$$
U_{A}, U_{B} \rightarrow e^{+i \sigma_{3} \Phi_{1}-\sigma_{0} \Phi_{2}} .
$$

To sum up, in the naive continuum limit the MDH model is given by

$$
H_{\mathrm{MDH}}=\left(\begin{array}{cc}
0 & D_{\mathrm{MDH}} \\
D_{\mathrm{MDH}}^{\dagger} & 0
\end{array}\right), \quad D_{\mathrm{MDH}}=i \sigma_{\mu}\left(\partial_{\mu}+A_{\mu}\right),
$$

where the vector potential $\mathbf{A}$ is randomly distributed according to

$$
\overline{A_{\mu}(\boldsymbol{x}) A_{\nu}(\boldsymbol{y})}=g_{A} \delta_{\mu \nu} \delta(\boldsymbol{x}-\boldsymbol{y}), \quad \mu, \nu=1,2 .
$$

\section{DOS AND FIELD THEORY}

\section{A. Generalities}

The DOS $\nu(\varepsilon)$ per unit energy and per unit volume $L^{2}$ for the single-particle Hamiltonian $H$ with eigenvalues $\varepsilon_{n}$ is defined by

$$
\begin{aligned}
\nu(\varepsilon) & :=\frac{1}{L^{2}} \sum_{n} \delta\left(\varepsilon-\varepsilon_{n}\right)=\frac{1}{L^{2}} \sum_{n} \lim _{\eta \rightarrow 0} \frac{1}{\pi} \operatorname{Im}\left(\frac{1}{\varepsilon-\varepsilon_{n}-i \eta}\right) \\
& =\lim _{\eta \rightarrow 0} \frac{1}{\pi L^{2}} \sum_{n} \frac{\eta}{\left(\varepsilon-\varepsilon_{n}\right)^{2}+\eta^{2}} .
\end{aligned}
$$

With $\eta$ kept small but finite, Eq. (3.1) defines the smeared DOS. The LDOS $\nu(\varepsilon ; \boldsymbol{x})$ is defined in terms of the exact normalized eigenfunctions $\Psi_{n}$ of $H$ by

$$
\nu(\varepsilon ; \boldsymbol{x}):=\lim _{\eta \rightarrow 0} \frac{1}{\pi} \sum_{n} \frac{\eta\left|\Psi_{n}(\boldsymbol{x})\right|^{2}}{\left(\varepsilon-\varepsilon_{n}\right)^{2}+\eta^{2}} .
$$

When the single-particle Hamiltonian $H$ is one member of a statistical ensemble, it is commonly held that the smeared DOS is self-averaging in the thermodynamic limit. On the other hand, critical statistical fluctuations of the wave function amplitudes at $\boldsymbol{x}$ open up the possibility that the (smeared) LDOS is broadly distributed in the thermodynamic limit.

The computation of the (smeared) LDOS in field theory is very natural as it reduces to the calculation of the expectation value of a local composite operator. For example, the smeared LDOS can be equivalently well represented by fermionic or bosonic path integrals

$$
\begin{aligned}
\nu(\varepsilon-i \eta ; \boldsymbol{x}) & =-\frac{1}{\pi} \operatorname{Im} \frac{\int \mathcal{D}[\bar{\psi}, \psi] \psi(\boldsymbol{x}) \bar{\psi}(\boldsymbol{x}) \exp \left[-\int d^{2} \boldsymbol{r} \bar{\psi}(\boldsymbol{r})(H-\varepsilon+i \eta) \psi(\boldsymbol{r})\right]}{\int \mathcal{D}[\bar{\psi}, \psi] \exp \left[-\int d^{2} \boldsymbol{r} \bar{\psi}(\boldsymbol{r})(H-\varepsilon+i \eta) \psi(\boldsymbol{r})\right]} \\
& =-\frac{1}{\pi} \operatorname{Im} \frac{\int \mathcal{D}[\bar{\beta}, \beta] \beta(\boldsymbol{x}) \bar{\beta}(\boldsymbol{x}) \exp \left[-\int d^{2} \boldsymbol{r} \bar{\beta}(\boldsymbol{r})(H-\varepsilon+i \eta) \beta(\boldsymbol{r})\right]}{\int \mathcal{D}[\bar{\beta}, \beta] \exp \left[-\int d^{2} \boldsymbol{r} \bar{\beta}(\boldsymbol{r})(H-\varepsilon+i \eta) \beta(\boldsymbol{r})\right]},
\end{aligned}
$$


respectively. Here $\bar{\psi}$ and $\psi$ denote two independent Grassmann fields whereas $\bar{\beta}$ denotes the complex conjugate of the complex-valued field $\beta$.

A SUSY representation for the smeared DOS can be achieved by combining the first with the second line of Eq. (3.3):

$$
\begin{aligned}
\nu(\varepsilon-i \eta) & =-\frac{1}{\pi L^{2}} \operatorname{Im} \int \mathcal{D}[\bar{\psi}, \psi, \bar{\beta}, \beta] \int d^{2} \boldsymbol{x} \psi(\boldsymbol{x}) \bar{\psi}(\boldsymbol{x}) e^{-\int d^{2} \boldsymbol{r}[\bar{\psi}(\boldsymbol{r})(H-\varepsilon+i \eta) \psi(\boldsymbol{r})+\bar{\beta}(\boldsymbol{r})(H-\varepsilon+i \eta) \beta(\boldsymbol{r})]} \\
& =-\frac{1}{2 \pi L^{2}} \operatorname{Im}\left\langle\int d^{2} \boldsymbol{x}[\psi(\boldsymbol{x}) \bar{\psi}(\boldsymbol{x})+\beta(\boldsymbol{x}) \bar{\beta}(\boldsymbol{x})]\right\rangle_{Z_{\mathrm{SUSY}}(\varepsilon-i \eta)}
\end{aligned}
$$

where $\langle(\cdots)\rangle_{Z_{\text {SUSY }}(\varepsilon-i \eta)}$ denotes averaging with the SUSY partition functions defined by

$$
\begin{aligned}
Z_{\mathrm{SUSY}}(\varepsilon-i \eta):= & \int \mathcal{D}[\bar{\psi}, \psi, \bar{\beta}, \beta] \exp \left\{-\int d^{2} \boldsymbol{r}[\bar{\psi}(\boldsymbol{r})(H-\varepsilon\right. \\
& +i \eta) \psi(\boldsymbol{r})+\bar{\beta}(\boldsymbol{r})(H-\varepsilon+i \eta) \beta(\boldsymbol{r})]\} .
\end{aligned}
$$

Alternatively, one can replicate either line of Eq. (3.3) to obtain a fermion (boson) replica representation of the DOS.

By assuming that disorder averaging restores translation invariance, we infer that the smeared LDOS $\nu(\varepsilon-i \eta ; \boldsymbol{x})$ and the smeared DOS $\nu(\varepsilon-i \eta)$ share the same mean value as statistical variables. However, their distributions are likely to be very different in the thermodynamic limit $L \rightarrow \infty$ at some fixed energy $\varepsilon-i \eta$ as the former is expected to be broadly distributed whereas the latter is expected to be selfaveraging. From now on, we will refer to the disorder average DOS as the DOS having in mind the expectation that the DOS is self-averaging.

\section{B. SUSY partition function for the LDOS in the HWK model}

Needed is a SUSY partition function from which the LDOS in the HWK model can be represented by the expectation value of some local composite operator. To construct a convenient SUSY partition function we choose a realization of the disorder $A_{0}, A_{1}, A_{2}$, and $A_{3}$ in Eq. (2.15) and define the fermionic partition function

$$
\begin{gathered}
Z^{(f)}:=\int \mathcal{D}\left[\bar{\psi}_{A}, \psi_{A}, \bar{\psi}_{B}, \psi_{B}\right] e^{-\int d^{2} r\left[\mathcal{L}_{A B}^{(f)}+\mathcal{L}_{B A}^{(f)}+\mathcal{L}_{\varepsilon}^{(f)}\right]}, \\
\mathcal{L}_{A B}^{(f)}=\bar{\psi}_{A} D_{\mathrm{HWK}} \psi_{B}, \\
\mathcal{L}_{B A}^{(f)}=\bar{\psi}_{B} D_{\mathrm{HWK}}^{\dagger} \psi_{A}, \\
\mathcal{L}_{\varepsilon}^{(f)}=-\varepsilon\left(\bar{\psi}_{A} \psi_{A}+\bar{\psi}_{B} \psi_{B}\right) .
\end{gathered}
$$

In view of the particle-hole symmetry, we will assume that $\varepsilon>0$ from now on and without loss of generality. The SUSY partition function is obtained by first, multiplying $Z^{(f)}$ by $Z^{(b)}$, whereby $Z^{(b)}$ follows from $Z^{(f)}$ after replacing all Grassmann fields $\bar{\psi}$ and $\psi$ by the complex-valued fields $\bar{\beta}$ and $\beta$, respectively, and second, by integrating over disorder. We are now going to perform three successive changes of path integral variables in the "matter" ( $\bar{\psi}$ 's, $\psi$ 's, $\bar{\beta}$ 's, and $\beta$ 's) sector. We will only specify the first two changes of variables in the fermion $(\psi)$ sector as it is understood that the same changes of variables apply to the boson $(\beta)$ sector. We will treat the fermion and boson sectors in an asymmetric way in the last step. Although these are symbolic manipulations, they can be justified by choosing appropriate unitary equivalent representations of $H_{\mathrm{HWK}}$ for the fermion and boson partition functions, respectively. ${ }^{27}$

First, introduce

$$
\bar{\psi}_{A}=: \psi_{A}^{*} \sigma_{1}, \quad \bar{\psi}_{B}=: \psi_{B}^{*} \sigma_{1},
$$

whereby $\psi_{A}$ and $\psi_{B}$ are left unchanged. Denote eigenstates of $\sigma_{3}$ according to

$$
\sigma_{3} \psi_{A \pm}=\mp \psi_{A \pm}, \quad \sigma_{3} \psi_{B \pm}=\mp \psi_{B \pm},
$$

say, and rewrite the disorder as

$$
A_{ \pm}:=A_{1} \pm i A_{2}, \quad M_{ \pm}:=A_{0} \pm i A_{3} .
$$

This gives the fermion Lagrangians

$$
\begin{aligned}
& \mathcal{L}_{A B}^{(f)}= i\left[\psi_{A-}^{*}\left(\partial_{+}+A_{+}\right) \psi_{B-}+\psi_{A+}^{*}\left(\partial_{-}+A_{-}\right) \psi_{B+}\right] \\
&+i\left(\psi_{A-}^{*} M_{+} \psi_{B+}+\psi_{A+}^{*} M_{-} \psi_{B-}\right), \\
& \mathcal{L}_{B A}^{(f)}= i\left[\psi_{B-}^{*}\left(\partial_{+}-A_{+}\right) \psi_{A-}+\psi_{B+}^{*}\left(\partial_{-}-A_{-}\right) \psi_{A+}\right] \\
&-i\left(\psi_{B-}^{*} M_{-} \psi_{A+}+\psi_{B+}^{*} M_{+} \psi_{A-}\right), \\
& \mathcal{L}_{\varepsilon}^{(f)}=-\varepsilon\left[\psi_{A-}^{*} \psi_{A+}+\psi_{B-}^{*} \psi_{B+}+(+\leftrightarrow-)\right],
\end{aligned}
$$

whereby $\partial_{ \pm}:=\partial_{1} \pm i \partial_{2}$. Their boson counterparts follow from substitution of the $\psi^{*}$ 's by the $\beta^{*}$ 's and of the $\psi^{\prime}$ 's by the $\beta$ 's.

Second, define 


$$
\begin{aligned}
& \psi_{A}^{*}=: \frac{1}{\sqrt{2 \pi} i} \psi_{1}^{*}, \quad \psi_{B}^{*}=: \frac{1}{\sqrt{2 \pi}} \psi_{2}^{*}, \\
& \psi_{A}=: \frac{1}{\sqrt{2 \pi} i} \psi_{2}, \quad \psi_{B}=: \frac{1}{\sqrt{2 \pi}} \psi_{1} .
\end{aligned}
$$

The rescaling of the fields is done for later convenience when computing operator product expansion (OPE). We also rescale the energy $\varepsilon \rightarrow 2 \pi \varepsilon$. This gives the fermion Lagrangians

$$
\begin{aligned}
\mathcal{L}_{A B}^{(f)}= & \frac{1}{2 \pi}\left[\psi_{1-}^{*}\left(\partial_{+}+A_{+}\right) \psi_{1-}+\psi_{1+}^{*}\left(\partial_{-}+A_{-}\right) \psi_{1+}\right] \\
& +\frac{1}{2 \pi}\left(\psi_{1-}^{*} M_{+} \psi_{1+}+\psi_{1+}^{*} M_{-} \psi_{1-}\right), \\
\mathcal{L}_{B A}^{(f)}= & \frac{1}{2 \pi}\left[\psi_{2-}^{*}\left(\partial_{+}-A_{+}\right) \psi_{2-}+\psi_{2+}^{*}\left(\partial_{-}-A_{-}\right) \psi_{2+}\right] \\
& -\frac{1}{2 \pi}\left(\psi_{2-}^{*} M_{-} \psi_{2+}+\psi_{2+}^{*} M_{+} \psi_{2-}\right), \\
\mathcal{L}_{\varepsilon}^{(f)}= & \varepsilon\left(\psi_{1-}^{*} \psi_{2+}-\psi_{2-}^{*} \psi_{1+}+\psi_{1+}^{*} \psi_{2-}-\psi_{2+}^{*} \psi_{1-}\right) .
\end{aligned}
$$

Their boson counterparts follow from substitution of the $\psi^{*}$ 's by the $\beta^{*}$ 's and of the $\psi$ 's by the $\beta$ 's. Observe that to go from $\mathcal{L}_{A B}^{(f)}+\mathcal{L}_{A B}^{(b)}$ to $\mathcal{L}_{B A}^{(f)}+\mathcal{L}_{B A}^{(b)}$ it suffices to change by a sign the vector potential $\mathbf{A}$ and the scalar potential $A_{0}$, while leaving the sign of the mass potential $A_{3}$ unchanged.

This asymmetry between the two sublattices can be removed by performing the final change of path integral variables according to ${ }^{27}$

$$
\begin{aligned}
& \psi_{2}^{*} \rightarrow+\psi_{2}, \quad \psi_{2} \rightarrow+\psi_{2}^{*}, \\
& \beta_{2}^{*} \rightarrow+\beta_{2}, \quad \beta_{2} \rightarrow-\beta_{2}^{*},
\end{aligned}
$$

with all other fields unchanged. This gives the fermion Lagrangian

$$
\begin{aligned}
\mathcal{L}_{A B}^{(f)}+\mathcal{L}_{B A}^{(f)}= & \sum_{a=1,2} \frac{1}{2 \pi}\left[\psi_{a-}^{*}\left(\partial_{+}+A_{+}\right) \psi_{a-}\right. \\
& +\psi_{a+}^{*}\left(\partial_{-}+A_{-}\right) \psi_{a+}+\psi_{a-}^{*} M_{+} \psi_{a+} \\
& \left.+\psi_{a+}^{*} M_{-} \psi_{a-}\right],
\end{aligned}
$$

together with its boson counterparts obtained by substitution of the $\psi^{*}$ 's by the $\beta^{*}$ 's and of the $\psi$ 's by the $\beta$ 's:

$$
\begin{aligned}
\mathcal{L}_{A B}^{(b)}+\mathcal{L}_{B A}^{(b)}= & \sum_{a=1,2} \frac{1}{2 \pi}\left[\beta_{a-}^{*}\left(\partial_{+}+A_{+}\right) \beta_{a-}\right. \\
& +\beta_{a+}^{*}\left(\partial_{-}+A_{-}\right) \beta_{a+}+\beta_{a-}^{*} M_{+} \beta_{a+} \\
& \left.+\beta_{a+}^{*} M_{-} \beta_{a-}\right] .
\end{aligned}
$$

The asymmetry between sublattices is not completely removed, however, since it is disguised as a perturbation for the DOS with non-Hermitian appearance: ${ }^{49}$

$$
\begin{aligned}
\mathcal{L}_{\varepsilon}^{(f)} & =\varepsilon\left(\psi_{1-}^{*} \psi_{2+}^{*}-\psi_{2-} \psi_{1+}+\psi_{1+}^{*} \psi_{2-}^{*}-\psi_{2+} \psi_{1-}\right), \\
\mathcal{L}_{\varepsilon}^{(b)} & =-\varepsilon\left(\beta_{1-}^{*} \beta_{2+}^{*}+\beta_{2-} \beta_{1+}+\beta_{1+}^{*} \beta_{2-}^{*}+\beta_{2+} \beta_{1-}\right) .
\end{aligned}
$$

The "decoupling transformation" (2.20) now reads (the flavor index runs over $a=1,2$ )

$$
\begin{array}{ll}
\psi_{a-}^{*}=\psi_{a-}^{\prime *} e^{-i \Phi_{1}+\Phi_{2}}, & \psi_{a-}=e^{+i \Phi_{1}-\Phi_{2}} \psi_{a-}^{\prime}, \\
\psi_{a+}^{*}=\psi_{a+}^{\prime *} e^{+i \Phi_{1}+\Phi_{2}}, & \psi_{a+}=e^{-i \Phi_{1}-\Phi_{2}} \psi_{a+}^{\prime},
\end{array}
$$

in the fermion sector, and

$$
\begin{array}{ll}
\beta_{a-}^{*}=\beta_{a-}^{\prime *} e^{-i \Phi_{1}+\Phi_{2}}, & \beta_{a-}=e^{+i \Phi_{1}-\Phi_{2}} \beta_{a-}^{\prime}, \\
\beta_{a+}^{*}=\beta_{a+}^{\prime *} e^{+i \Phi_{1}+\Phi_{2}}, & \beta_{a+}=e^{-i \Phi_{1}-\Phi_{2}} \beta_{a+}^{\prime},
\end{array}
$$

in the boson sector. Under the chiral transformation (3.14) we note that the fermion Lagrangians become

$$
\begin{aligned}
\mathcal{L}_{A B}^{(f)}+\mathcal{L}_{B A}^{(f)}= & \frac{1}{2 \pi} \sum_{a=1,2}\left(\psi_{a-}^{\prime *} \partial_{+} \psi_{a-}^{\prime}+\psi_{a+}^{\prime *} \partial_{-} \psi_{a+}^{\prime}\right. \\
+ & \left.\psi_{a-}^{\prime *} M_{+} e^{-2 i \Phi_{1}} \psi_{a+}^{\prime}+\psi_{a+}^{\prime *} M_{-} e^{+2 i \Phi_{1}} \psi_{a-}^{\prime}\right), \\
\mathcal{L}_{\varepsilon}^{(f)}= & +\varepsilon e^{+2 \Phi_{2}}\left(\psi_{1-}^{\prime *} \psi_{2+}^{\prime *}+\psi_{1+}^{\prime *} \psi_{2-}^{\prime *}\right) \\
& -\varepsilon e^{-2 \Phi_{2}}\left(\psi_{2-}^{\prime} \psi_{1+}^{\prime}+\psi_{2+}^{\prime} \psi_{1-}^{\prime}\right),
\end{aligned}
$$

whereas the boson Lagrangians become

$$
\begin{aligned}
\mathcal{L}_{A B}^{(b)}+\mathcal{L}_{B A}^{(b)}= & \frac{1}{2 \pi} \sum_{a=1,2}\left(\beta_{a-}^{\prime *} \partial_{+} \beta_{a-}^{\prime}+\beta_{a+}^{\prime *} \partial_{-} \beta_{a+}^{\prime}\right. \\
& \left.+\beta_{a-}^{\prime *} M_{+} e^{-2 i \Phi_{1}} \beta_{a+}^{\prime}+\beta_{a+}^{\prime *} M_{-} e^{+2 i \Phi_{1}} \beta_{a-}^{\prime}\right), \\
\mathcal{L}_{\varepsilon}^{(b)}= & -\varepsilon e^{+2 \Phi_{2}}\left(\beta_{1-}^{\prime *} \beta_{2+}^{\prime *}+\beta_{1+}^{\prime *} \beta_{2-}^{\prime *}\right) \\
& -\varepsilon e^{-2 \Phi_{2}}\left(\beta_{2-}^{\prime} \beta_{1+}^{\prime}+\beta_{2+}^{\prime} \beta_{1-}^{\prime}\right) .
\end{aligned}
$$

The complex-valued random masses $M_{ \pm}$couple to the transversal component $\Phi_{1}$ of the random vector potential through a bounded phase factor $\exp \left( \pm 2 i \Phi_{1}\right)$ whereas they decouple from the longitudinal component $\Phi_{2}$ of the random vector potential. Conversely, the energy term couples to the longitudinal component $\Phi_{2}$ of the random vector potential through an unbounded exponential factor $\exp \left( \pm 2 \Phi_{2}\right)$ whereas it decouples from the transversal component $\Phi_{1}$ of the random vector potential. We conclude, with the help of the decoupling transformation (3.14), that the random vector potential enters in the energy perturbation $\mathcal{L}_{\varepsilon}^{(f)}+\mathcal{L}_{\varepsilon}^{(b)}$ as it does in the squared amplitude of the zero mode of the operator $D_{\mathrm{MDH}}^{\dagger}$ in Eq. (2.22) - namely, through the unbounded exponential factors $\exp \left( \pm 2 \Phi_{2}\right)$. We shall see that these ex- 
ponential factors dictate the dependence on $\varepsilon$ of the DOS in the close vicinity of the band center. ${ }^{50}$

The SUSY partition function for the HWK model that we will choose to work with treats quantum mechanical (through the SUSY matter fields $\psi$ and $\beta$ ) and disorder (through the random vector and mass potentials) averaging on an equal footing:

$$
\begin{aligned}
Z=\int \mathcal{D}\left[A_{ \pm}, M_{ \pm}, \psi_{+}^{*}, \psi_{\mp}, \beta_{+}^{*}, \beta_{\mp}\right] e^{-\int d^{2} r \mathcal{L}}, \\
\mathcal{L}=\frac{1}{2 g_{A}} A_{+} A_{-}+\frac{1}{2 g_{M}} M_{+} M_{-} \\
\quad+\mathcal{L}_{A B}^{(f)}+\mathcal{L}_{B A}^{(f)}+\mathcal{L}_{\varepsilon}^{(f)}+\mathcal{L}_{A B}^{(b)}+\mathcal{L}_{B A}^{(b)}+\mathcal{L}_{\varepsilon}^{(b)},
\end{aligned}
$$

where $\mathcal{L}_{A B}^{(f / b)}+\mathcal{L}_{B A}^{(f / b)}+\mathcal{L}_{\varepsilon}^{(f / b)}$ are given by Eq. (3.13). Correspondingly, the SUSY partition function for the MDH model is obtained from Eq. (3.16) by switching off the random masses $M_{ \pm}$, i.e., setting the variance $g_{M}=0$. This SUSY partition function is critical at the band center $\varepsilon=0$ as follows from the decoupling transformation (3.14). Indeed, it is then given by

$$
\begin{gathered}
Z_{*}=\int \mathcal{D}\left[\bar{\alpha}, \alpha, \Phi_{1}, \Phi_{2}, \psi_{\mp}^{\prime *}, \psi_{\mp}^{\prime}, \beta_{\mp}^{\prime *}, \beta_{\mp}^{\prime}\right] e^{-\int d^{2} r \mathcal{L}_{*}}, \\
\mathcal{L}_{*}=\bar{\alpha} \partial^{2} \alpha+\frac{1}{2 g_{A}}\left(\partial_{\mu} \Phi_{1}\right)^{2}+\frac{1}{2 g_{A}}\left(\partial_{\mu} \Phi_{2}\right)^{2} \\
+\mathcal{L}_{A B}^{(f)}+\mathcal{L}_{B A}^{(f)}+\mathcal{L}_{A B}^{(b)}+\mathcal{L}_{B A}^{(b)},
\end{gathered}
$$

where $\mathcal{L}_{A B}^{(f)}+\mathcal{L}_{B A}^{(f)}$ and $\mathcal{L}_{A B}^{(b)}+\mathcal{L}_{B A}^{(b)}$ follow from Eqs. (3.15a) and (3.15b) with the random masses $M_{ \pm}$switched off, respectively. We have exponentiated the Jacobian that arises from expressing the vector potential in terms of its longitudinal and transversal components with the help of fermionic ghosts $\bar{\alpha}$ and $\alpha$. In doing so we ensure that the SUSY partition function remains unity. The Fujikawa Jacobian arising from the decoupling transformation in the fermionic sector cancels with that from the bosonic sector.

It was shown in Ref. 37 that Eq. (3.17) defines a CFT for each value of the marginal coupling constant $g_{A}$. The full operator content was constructed explicitly; i.e., anomalous scaling dimensions of all local operators that can be built out of the $\psi$ 's and $\beta$ 's were calculated. Furthermore, it was established that this CFT realizes a $\mathrm{gl}(2 \mid 2)$ current algebra. This property allows the explicit construction of primary fields realizing one- and two-dimensional representations, say, of the current algebra. The anomalous scaling dimensions of primary fields carrying multiples of the $\mathrm{U}(1)$ gauge charge of 2 were shown to be unbounded from below, related to the "Cooper-like" terms entering $\mathcal{L}_{\varepsilon}^{(b)}$, and responsible for the multifractality of the zero mode. We are going to combine these results with a method pioneered by Carpentier and Le Doussal in Ref. 35 to compute the DOS for the MDH model. We will then show that the anomalous scaling dimensions of the family of Cooper-like terms that govern both multifractality of the zero mode and the DOS in the MDH model also control the DOS in the HWK model. To this end, we will make use of the fact, demonstrated by Guruswamy et al. in Ref. 27, that Eq. (3.16) defines a nearly conformal field theory in which the coupling constant $g_{A}$ is marginally relevant (in the sense that it grows logarithmically with length) and the coupling constant $g_{M}$ is exactly marginal.

\section{DOS FOR $\boldsymbol{H}_{\mathrm{MDH}}$}

In this section, we are going to compute the DOS in the MDH model. We shall develop an approximation that, presumably, becomes exact in the limit $\varepsilon \rightarrow 0$ and in which the DOS for the MDH model reduces to the DOS for a single species of Dirac fermion subjected to a real-valued random vector potential. The cornerstone of our calculation is to distinguish between the annealed and quenched average values of the dynamical exponent in very much the same way as one must distinguish between the scaling with system size of the average and typical values of the normalization of zero modes. In this way we will have established a one-to-one correspondence between the power-law dependence of the DOS and the multifractal spectrum of zero modes.

Our starting point is the critical theory (3.17) from which we extract the nonvanishing two-point functions

$$
\begin{gathered}
\left\langle\Phi_{2}(z, \bar{z}) \Phi_{2}(0)\right\rangle_{*}=-\frac{g_{A}}{2 \pi} \ln |z|, \\
\left\langle\psi_{a-}^{\prime}(z) \psi_{b-}^{\prime *}(0)\right\rangle_{*}=+\left\langle\psi_{b-}^{\prime *}(z) \psi_{a-}^{\prime}(0)\right\rangle_{*}=\frac{\delta_{a b}}{z}, \\
\left\langle\psi_{a+}^{\prime}(\bar{z}) \psi_{b+}^{\prime *}(0)\right\rangle_{*}=+\left\langle\psi_{b+}^{\prime *}(\bar{z}) \psi_{a+}^{\prime}(0)\right\rangle_{*}=\frac{\delta_{a b}}{\bar{z}}, \\
\left\langle\beta_{a-}^{\prime}(z) \beta_{b-}^{\prime *}(0)\right\rangle_{*}=-\left\langle\beta_{b-}^{\prime *}(z) \beta_{a-}^{\prime}(0)\right\rangle_{*}=\frac{\delta_{a b}}{z}, \\
\left\langle\beta_{a+}^{\prime}(\bar{z}) \beta_{b+}^{\prime *}(0)\right\rangle_{*}=-\left\langle\beta_{b+}^{\prime *}(\bar{z}) \beta_{a+}^{\prime}(0)\right\rangle_{*}=\frac{\delta_{a b}}{\bar{z}}, \\
a, b=1,2 .
\end{gathered}
$$

A point $\boldsymbol{r}=\left(r_{1}, r_{2}\right) \in \mathbb{R}^{2}$ in the two-dimensional Euclidean plane is hereby parametrized by the complex variables $z$ $:=r_{1}+i r_{2}$ and $\bar{z}:=r_{1}-i r_{2}$, respectively.

The disorder-average-smeared DOS in the MDH model is to be calculated by adding to the critical theory (3.17) the perturbation $\mathcal{L}_{\varepsilon-i \eta}:=-(\varepsilon-i \eta) \mathcal{O}_{\varepsilon-i \eta}$, where

$$
\begin{aligned}
\mathcal{O}_{\varepsilon-i \eta}:= & +e^{+2 \Phi_{2}}\left(\mathcal{B}_{12+}+\mathcal{B}_{21+}-\mathcal{F}_{12+}-\mathcal{F}_{21+}\right) \\
& +e^{-2 \Phi_{2}}\left(\mathcal{B}_{12-}+\mathcal{B}_{21-}+\mathcal{F}_{12-}+\mathcal{F}_{21-}\right)
\end{aligned}
$$

and we have defined the composite operators

$$
\begin{array}{ll}
\mathcal{F}_{12+}:=\psi_{1+}^{\prime *} \psi_{2-}^{\prime *}, & \mathcal{F}_{21+}:=\psi_{1-}^{\prime *} \psi_{2+}^{\prime *}, \\
\mathcal{F}_{12-}:=\psi_{2-}^{\prime} \psi_{1+}^{\prime}, & \mathcal{F}_{21-}:=\psi_{2+}^{\prime} \psi_{1-}^{\prime},
\end{array}
$$

in the fermionic sector [see Eq. (3.15a)], and the composite operators 


$$
\begin{array}{ll}
\mathcal{B}_{12+}:=\beta_{1+}^{\prime *} \beta_{2-}^{\prime *}, & \mathcal{B}_{21+}:=\beta_{1-}^{\prime *} \beta_{2+}^{\prime *}, \\
\mathcal{B}_{12-}:=\beta_{2-}^{\prime} \beta_{1+}^{\prime}, & \mathcal{B}_{21-}:=\beta_{2+}^{\prime} \beta_{1-}^{\prime},
\end{array}
$$

in the bosonic sector [see Eq. (3.15b)]. Furthermore, in the definition of the DOS (3.4a) the operator to be averaged over is now given by

$$
\begin{aligned}
\mathcal{O}_{\nu}= & +e^{+2 \Phi_{2}}\left(\mathcal{B}_{12+}+\mathcal{B}_{21+}+\mathcal{F}_{12+}+\mathcal{F}_{21+}\right) \\
& +e^{-2 \Phi_{2}}\left(\mathcal{B}_{12-}+\mathcal{B}_{21-}-\mathcal{F}_{12-}-\mathcal{F}_{21-}\right) .
\end{aligned}
$$

Observe that $\mathcal{O}_{\nu}$ has the same anomalous scaling dimension as the energy perturbation $\mathcal{O}_{\varepsilon-i \eta}$.

Our strategy is, first, to derive a differential (CallanSymanzik) equation obeyed by the DOS that encodes its change under the infinitesimal rescaling of the lattice constant

$$
\mathfrak{a} \rightarrow \mathfrak{a}^{\prime}=(1+d l) \mathfrak{a} .
$$

Second, we must solve the Callan-Symanzik equation obeyed by the DOS.

In this spirit, we modify the critical action $S_{*}$ defined by Eq. (3.17) by adding to it the energy perturbation and any other operator $\mathcal{O}_{\iota}$ needed to ensure invariance under infinitesimal rescaling of the lattice spacing. In other words, we seek a family of local composite operators $\left\{\mathcal{O}_{\iota}\right\}$ such that

$$
S:=S_{*}-\int d^{2} \boldsymbol{r}\left(\lambda_{\varepsilon} \mathfrak{a}^{x_{\varepsilon}-2} \mathcal{O}_{\varepsilon-i \eta}+\sum_{\iota} \lambda_{\iota} \mathfrak{a}^{x_{\iota}-2} \mathcal{O}_{\iota}\right)
$$

is invariant under infinitesimal rescaling of the lattice spacing. The dimensionless coupling constants $\lambda_{\iota}$ are scale $(l)$ dependent and the anomalous scaling dimensions $x_{\iota}$ of the composite operators $\mathcal{O}_{\iota}$ are to be calculated at criticality. Invariance of the right-hand side under infinitesimal rescaling of the lattice spacing is equivalent to demanding that the family of composite operators labeled by $\varepsilon$ and $\iota$ is closed under the OPE. Although the range of $\iota$ is always infinite (for one, composite operators can always be point split and Taylor expanded), perturbation of a unitary CFT simplifies greatly in that the family $\left\{\mathcal{O}_{\iota}\right\}$ contains no more than a finite number of relevant $\left(0<x_{\iota}<2\right)$ operators. In practice, it is then sufficient to truncate the family $\left\{\mathcal{O}_{\iota}\right\}$ to this finite subset of relevant operators if one is only interested in the effect of an arbitrary small perturbation to criticality. For a nonunitary CFT, as is Eq. (3.17), the situation is dramatically different, however, since $\mathcal{O}_{\varepsilon-i \eta}$ induces under repeated OPE infinitely many relevant $\left(x_{\iota}<2\right)$ operators. After selection of the family $\left\{\mathcal{O}_{\iota}\right\}$, the DOS $\nu(\varepsilon)$ obeys the Callan-Symanzik equation

$$
\begin{gathered}
0=\left(\beta_{\lambda_{\varepsilon}} \frac{\partial}{\partial \lambda_{\varepsilon}}+\sum_{\iota} \beta_{\lambda_{\iota}} \frac{\partial}{\partial \lambda_{\iota}}-x_{\varepsilon}\right) \nu(\varepsilon), \\
\beta_{\lambda_{\varepsilon}}=\frac{d \lambda_{\varepsilon}}{d l}, \quad \beta_{\lambda_{\iota}}=\frac{d \lambda_{\iota}}{d l} .
\end{gathered}
$$

We then go on to solve Eq. (4.6) in some approximate scheme.

\section{A. Operator content at criticality and RG equations}

At criticality and through OPE with itself, the energy perturbation $\mathcal{O}_{\varepsilon-i \eta}$ induces local composite operators of the form

$$
\begin{aligned}
\mathcal{O}_{\mathbf{m}, \mathbf{n}}= & \left(\mathcal{F}_{12+}\right)^{m_{12+}}\left(\mathcal{F}_{21+}\right)^{m_{21+}}\left(\mathcal{B}_{12+}\right)^{n_{12+}}\left(\mathcal{B}_{21+}\right)^{n_{21+}}\left(\mathcal{F}_{12-}\right)^{m_{12-}}\left(\mathcal{F}_{21-}\right)^{m_{21-}}\left(\mathcal{B}_{12-}\right)^{n_{12-}}\left(\mathcal{B}_{21-}\right)^{n_{21-}} \\
& \times \exp \left[2\left(m_{12+}+m_{21+}+n_{12+}+n_{21+}-m_{12-}-m_{21-}-n_{12-}-n_{21-}\right) \Phi_{2}\right], \quad \forall \mathbf{m}, \mathbf{n} \in \mathbb{N}^{4} .
\end{aligned}
$$

It can be shown along the lines of Refs. 36 and 37 that $\mathcal{O}_{\mathbf{m}, \mathbf{n}}$ carries the anomalous scaling dimension

$$
\begin{aligned}
x_{\mathbf{m}, \mathbf{n}}= & \left(m_{12+}-m_{12-}\right)^{2}+\left(m_{21+}-m_{21-}\right)^{2}+\left|n_{12+}-n_{12-}\right|+\left|n_{21+}-n_{21-}\right| \\
& -\frac{g_{A}}{\pi}\left[\left(m_{12+}-m_{12-}\right)+\left(m_{21+}-m_{21-}\right)+\left(n_{12+}-n_{12-}\right)+\left(n_{21+}-n_{21-}\right)\right]^{2},
\end{aligned}
$$

which should be contrasted with its engineering dimension $m_{12+}+m_{12-}+m_{21+}+m_{21-}+n_{12+}+n_{12-}+n_{21+}+n_{21-}$. Observe the difference in the contributions to the anomalous scaling dimensions between the fermion and boson sectors. Fermi statistics demands point splitting when taking the product of Grassmann variables. This leads to the quadratic dependence on components of $\mathbf{m}$ in Eq. (4.7b) when $g_{A}$ $=0$. Boson statistics accommodates arbitrary positive integer powers of complex-valued fields. This leads to the linear dependence on components of $\mathbf{n}$ in Eq. (4.7b) when $g_{A}=0$. The effect of disorder is thus much more potent in the boson sector than it is in the fermion sector since the positive term, which is linear in $\mathbf{n}$ when $g_{A}=0$, is always dominated by the negative term, which is quadratic in $\mathbf{n}$ when $g_{A}>0$, for sufficiently large $|\mathbf{n}|$. The boson sector of the SUSY theory conspires with the unboundedness of the exponential factor $\exp \left(2 \Phi_{2}\right)$ that enters in zero modes to produce infinitely many operators with negative anomalous scaling dimensions.

Alternatively, the difference between fermion and boson sectors can be deduced by noting that the boson sector is responsible for the normalization factor in correlation functions. Suppose that we perform an expansion of some correlation function in powers of $\exp \left( \pm 2 \Phi_{2}\right)$ without using the 
replica or SUSY method. There are contributions to this expansion that originate from the numerator and from the denominator of the correlation function. Perform term-by-term disorder averaging. It was shown in the context of the $2 \mathrm{D}$ random phase $X Y$ model in Ref. 41 that (i) the counterpart to the contribution from the boson (fermion) sector to the anomalous scaling dimension (4.7b) arises from disorder averaging over the contributions from the denominator (numerator) to the perturbative expansion in powers of $\exp \left( \pm 2 \Phi_{2}\right)$ and (ii) disorder averaging over the perturbative expansion in powers of $\exp \left( \pm 2 \Phi_{2}\right)$ leads to the breakdown of perturbation theory. The lesson from the 2D random phase $X Y$ model is that we cannot truncate the family $\left\{\mathcal{O}_{\mathbf{m}, \mathbf{n}}\right\}$ to some finite subset. We shall nevertheless argue that the family $\left\{\mathcal{O}_{\mathbf{m}, \mathbf{n}}\right\}$ can be truncated to an infinite but countable subset when computing the DOS for the MDH model.

To this end, we will again rely on the lessons to be drawn from a perturbative expansion of correlation functions in powers of $\exp \left( \pm 2 \Phi_{2}\right)$. To $N$ th order in powers of $\exp \left( \pm 2 \Phi_{2}\right)$ the most singular contributions to the disorder average are associated with terms proportional to $\exp$ $\left( \pm 2 N \Phi_{2}\right)$. For given

$$
\begin{aligned}
N= & m_{12+}+m_{21+}+n_{12+}+n_{21+} \\
& -m_{12-}-m_{21-}-n_{12-}-n_{21-},
\end{aligned}
$$

the most relevant operators of the form (4.7a) are given by

$$
\begin{gathered}
\left(\mathcal{B}_{12 \pm}\right)^{n}\left(\mathcal{B}_{21 \pm}\right)^{N-n} e^{ \pm 2 N \Phi_{2}}, \quad N \geqslant n \geqslant 0, \\
\left(\mathcal{F}_{12 \pm}\right)\left(\mathcal{B}_{12 \pm}\right)^{n}\left(\mathcal{B}_{21 \pm}\right)^{N-1-n} e^{ \pm 2 N \Phi_{2}}, \quad N-1 \geqslant n \geqslant 0, \\
\left(\mathcal{F}_{21 \pm}\right)\left(\mathcal{B}_{12 \pm}\right)^{n}\left(\mathcal{B}_{21 \pm}\right)^{N-1-n} e^{ \pm 2 N \Phi_{2}}, \quad N-1 \geqslant n \geqslant 0, \\
\left(\mathcal{F}_{12 \pm}\right)\left(\mathcal{F}_{21 \pm}\right)\left(\mathcal{B}_{12 \pm}\right)^{n}\left(\mathcal{B}_{21 \pm}\right)^{N-2-n} e^{ \pm 2 N \Phi_{2}}, \quad N-2 \geqslant n \geqslant 0,
\end{gathered}
$$

with the $(2 \times 4 N)$-fold degenerate anomalous scaling dimension

$$
x_{N}=N-\frac{g_{A}}{\pi} N^{2} .
$$

The $(2 \times 4 N)$-fold degeneracy of operators with the anomalous scaling dimension (4.10) suggests that we truncate the family (4.7a) to the subset

$$
\begin{aligned}
\mathcal{A}_{+; N}:= & \frac{e^{+2 N \Phi_{2}}}{N !}\left[\left(\mathcal{B}_{12+}+\mathcal{B}_{21+}\right)^{N}\right. \\
& -N\left(\mathcal{F}_{12+}+\mathcal{F}_{21+}\right)\left(\mathcal{B}_{12+}+\mathcal{B}_{21+}\right)^{N-1} \\
& \left.+N(N-1) \mathcal{F}_{12+} \mathcal{F}_{21+}\left(\mathcal{B}_{12+}+\mathcal{B}_{21+}\right)^{N-2}\right],
\end{aligned}
$$

$$
\begin{aligned}
\mathcal{A}_{-; N} & :=\frac{e^{-2 N \Phi_{2}}}{N !}\left[\left(\mathcal{B}_{12-}+\mathcal{B}_{21-}\right)^{N}\right. \\
& +N\left(\mathcal{F}_{12-}+\mathcal{F}_{21-}\right)\left(\mathcal{B}_{12-}+\mathcal{B}_{21-}\right)^{N-1} \\
& \left.+N(N-1) \mathcal{F}_{12-} \mathcal{F}_{21-}\left(\mathcal{B}_{12-}+\mathcal{B}_{21-}\right)^{N-2}\right],
\end{aligned}
$$

labeled by $0<N \in \mathbb{N}$ and \pm . Be aware of the sign difference on the second lines of Eqs. (4.11a) and (4.11b), respectively. The choice $\left\{\mathcal{A}_{ \pm ; N}\right\} \subset\left\{\mathcal{O}_{\mathbf{m}, \mathbf{n}}\right\}$ is dictated by the algebraic structure $\mathrm{gl}(2 \mid 2)$ of the critical theory (3.17). Indeed, by construction, the left-hand side of

$$
\begin{aligned}
\frac{e^{ \pm 2 N \Phi_{2}}}{N !}\left(\mathcal{B}_{12 \pm}+\mathcal{B}_{21 \pm} \mp \mathcal{F}_{12 \pm} \mp \mathcal{F}_{21 \pm}\right)^{N} \\
=+\frac{e^{ \pm 2 N \Phi_{2}}}{N !}\left(\mathcal{B}_{12 \pm}+\mathcal{B}_{21 \pm}\right)^{N} \\
\quad \mp N \frac{e^{ \pm 2 N \Phi_{2}}}{N !}\left(\mathcal{F}_{12 \pm}+\mathcal{F}_{21 \pm}\right)\left(\mathcal{B}_{12 \pm}+\mathcal{B}_{21 \pm}\right)^{N-1} \\
\quad+N(N-1) \frac{e^{ \pm 2 N \Phi_{2}}}{N !} \mathcal{F}_{12 \pm} \mathcal{F}_{21 \pm}\left(\mathcal{B}_{12 \pm}+\mathcal{B}_{21 \pm}\right)^{N-2} \\
\quad+\cdots
\end{aligned}
$$

is a SUSY singlet [though not a $\mathrm{gl}(2 \mid 2)$ singlet]. Contributions to the binomial expansion of the left-hand side are related to higher-dimensional irreducible representations of $\operatorname{gl}(2 \mid 2) .{ }^{37}$ Similarly, by taking the $N$ th power of the operator $\mathcal{O}_{\nu}$ in Eq. (4.3) we can construct the counterparts $\mathcal{A}_{\nu \pm ; N}$ to $\mathcal{A}_{ \pm ; N}$ that obey the same closed OPE's and share the same anomalous scaling dimensions. The consistency of the choice (4.11) is guaranteed by the fact that the OPE's (see Appendix A)

$$
\begin{aligned}
& \mathcal{A}_{+; N}(z, \bar{z}) \mathcal{A}_{+; N^{\prime}}(w, \bar{w}) \\
& =|z-w|^{x_{N+N^{\prime}}-x_{N}-x_{N^{\prime}}}\left(\begin{array}{c}
N+N^{\prime} \\
N
\end{array}\right) \mathcal{A}_{+; N+N^{\prime}}(w, \bar{w})+\cdots, \\
& 0<N, N^{\prime} \in \mathbb{N}, \\
& \mathcal{A}_{-; N}(z, \bar{z}) \mathcal{A}_{-; N^{\prime}}(w, \bar{w}) \\
& =|z-w|^{x_{N+N^{\prime}}-x_{N}-x_{N^{\prime}}}\left(\begin{array}{c}
N+N^{\prime} \\
N
\end{array}\right) \mathcal{A}_{-; N+N^{\prime}}(w, \bar{w})+\cdots, \\
& 0<N, N^{\prime} \in \mathbb{N},
\end{aligned}
$$




$$
\begin{aligned}
& \mathcal{A}_{+; N_{+}}(z, \bar{z}) \mathcal{A}_{-; N_{-}}(w, \bar{w})
\end{aligned}
$$

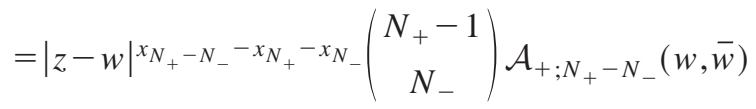

$$
\begin{aligned}
& +\cdots, \quad 0<N_{-}<N_{+} \in \mathbb{N}, \\
& \mathcal{A}_{-; N_{-}}(z, \bar{z}) \mathcal{A}_{+; N_{+}}(w, \bar{w})
\end{aligned}
$$

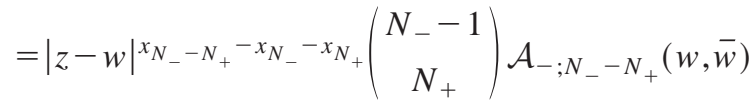

$$
\begin{aligned}
& +\cdots, \quad 0<N_{+}<N_{-} \in \mathbb{N},
\end{aligned}
$$

are closed within the subset $\left\{\mathcal{A}_{ \pm ; N}\right\} \subset\left\{\mathcal{O}_{\mathbf{m}, \mathbf{n}}\right\}$. Equation (4.13) is the most important technical result of this section. With the choice (4.11), Eq. (4.5) is approximated by

$$
\begin{gathered}
S=S_{*}-\sum_{s= \pm} \sum_{N_{s}=1}^{\infty} Y_{s ; N_{s}} \mathfrak{a}^{x_{s ; N_{s}}-2} \int d^{2} \boldsymbol{r} \mathcal{A}_{s ; N_{s}}, \\
x_{s ; N_{s}}=x_{N_{s}}=N_{s}-\frac{g_{A}}{\pi}\left(N_{s}\right)^{2} .
\end{gathered}
$$

Furthermore, by neglecting processes of "charge annihilation" as encoded by Eqs. (4.13c) and (4.13d) and their extensions when $N_{+}=N_{-}$in favor of "charge fusion" as encoded by Eqs. (4.13a) and (4.13b) we obtain the counterpart

$$
\begin{aligned}
0 & =\left(\sum_{s= \pm} \sum_{N_{s}=1}^{\infty} \frac{d Y_{s ; N_{s}}}{d l} \frac{\partial}{\partial Y_{s ; N_{s}}}-x_{+; 1}\right) \nu(\varepsilon), \\
\frac{d Y_{s ; N_{s}}}{d l} & =\left(2-x_{s ; N_{s}}\right) Y_{s ; N_{s}}+\pi \sum_{N_{s}^{\prime}=1}^{N_{s}-1}\left(\begin{array}{c}
N_{s} \\
N_{s}^{\prime}
\end{array}\right) Y_{s ; N_{s}^{\prime} Y_{s ; N_{s}-N_{s}^{\prime}}}
\end{aligned}
$$

to the Callan-Symanzik equation (4.6). At last, initial conditions on the coupling constants (fugacities) $Y_{s ; N_{s}}$ are independent of $s= \pm$ and given by

$$
Y_{s ; N_{s}}(l=0)=\frac{\varepsilon-i \eta}{\mathfrak{a}^{x_{s ; 1}-2}} \delta_{1, N_{s}} .
$$

The justification for neglecting all annihilation processes is that this approximation preserves the freezing transition that characterizes both the multifractal spectrum of zero modes ${ }^{38}$ and, as we shall see below, the DOS. This approximation is expected to capture the leading singularity of the DOS close to the band center. Furthermore, this approximation is consistent with neglecting renormalization effects on $g_{A}$ induced by the energy perturbation (see Appendix A). At this level of approximation, the distinction between positive and negative charges is irrelevant to the Callan-Symanzik equation obeyed by the DOS. Equation (4.15) thus simplifies to

$$
0=\left(\sum_{N=1}^{\infty} \beta_{Y_{N}} \frac{\partial}{\partial Y_{N}}-x_{1}\right) \nu(\varepsilon),
$$

$$
\begin{gathered}
\beta_{Y_{N}}=\left(2-x_{N}\right) Y_{N}+\pi \sum_{N^{\prime}=1}^{N-1}\left(\begin{array}{c}
N \\
N^{\prime}
\end{array}\right) Y_{N^{\prime}} Y_{N-N^{\prime}}, \\
x_{N}=N-\frac{g_{A}}{\pi} N^{2}, \\
Y_{N}(l=0)=\frac{\varepsilon-i \eta}{\mathfrak{a}^{x_{1}-2}} \delta_{1, N} .
\end{gathered}
$$

It can be shown that Eq. (4.17) is also obeyed by the DOS for a single flavor of Dirac fermions coupled to a real-valued random vector potential once all annihilation processes in the RG analysis have been neglected. Furthermore, essentially the same $\beta$ functions for the fugacities in Eq. (4.17) encode a RG analysis of the probability distribution for the normalization

$$
\mathcal{Z}(L):=\int_{L} d^{2} \boldsymbol{r} e^{-2 \Phi_{2}(\boldsymbol{r})}
$$

of the squared zero mode amplitude in the MDH model provided one replaces the anomalous scaling dimension $x_{N}=N$ $-\left(g_{A} / \pi\right) N^{2}$ by $-\left(g_{A} / \pi\right) N^{2}$. This is most easily seen after (i) performing the decoupling transformation (3.14) on Eq. (3.4a) applied to $H_{\mathrm{MDH}}$, (ii) isolating in the source term all terms that couple to $\exp \left(-2 \Phi_{2}\right)$, (iii) ignoring the RG contributions from the SUSY matter fields, and (iv) identifying the energy $\varepsilon$ with $L^{-z_{A}}$ where $L$ is the system size and $z_{A}$ is the dynamical scaling exponent that relates scaling with respect to $L$ to scaling with respect to $\varepsilon$. We have thus explicitly related the transformation law obeyed by the DOS under rescaling to the multifractal properties of zero modes. Similar RG equations were derived by Carpentier and Le Doussal within a replicated approach in Refs. 35 and 44 in the context of the 2D random $X Y$ model and the freezing transition of the multifractal spectrum of zero modes in the random vector potential problem, respectively. We now turn to the analysis of the Callan-Symanzik equation (4.17).

\section{B. From the Callan-Symanzik equation to the KPP equation}

The Callan-Symanzik equation (4.17) reflects the transformation law under infinitesimal rescaling of the ultraviolet cutoff (lattice spacing) of the SUSY partition function

$$
\begin{gathered}
Z:=\int \mathcal{D}\left[\bar{\alpha}, \alpha, \Phi_{1}, \Phi_{2}, \psi^{\prime *}, \psi^{\prime}, \beta^{\prime *}, \beta^{\prime}\right] e^{-\int d^{2} \boldsymbol{r} \mathcal{L}}, \\
\mathcal{L}:=\mathcal{L}_{*}-\sum_{n=1}^{\infty} Y_{n} \mathfrak{a}^{x_{n}-2} \mathcal{A}_{n},
\end{gathered}
$$

where $\mathcal{L}_{*}$ is given by Eq. (3.17) and $\mathcal{A}_{n}$ is, say, defined by the right-hand side of Eq. (4.11b). As noted earlier [see Eq. (4.12)],

$$
\mathcal{A}_{n}=\frac{1}{n !}\left(\mathcal{A}_{1}\right)^{n}+\ldots
$$


to leading order in an expansion organized by the relevance of the operators occurring in OPE's. It is then tempting to think of the coupling constants (fugacities) $Y_{n}$ as being related to the cumulants of some probability distribution. ${ }^{41}$ Initially, this probability distribution is very narrow since only the first cumulant is nonvanishing. However, as the lattice rescaling parameter $l$ is increased, the cumulants grow; i.e., the probability distribution becomes broader. In the infrared limit $l \rightarrow \infty$ the ratios $Y_{n} /\left(Y_{1}\right)^{n}$ diverge. The asymptotic RG flow of the fugacities thus offers little information on the bulk of the underlying probability distribution. Hence, it would be desirable to recast the RG equations obeyed by the fugacities $Y_{n}$ in terms of their generating function and to follow the RG flow of the generating function instead of that of the cumulants it generates so as to obtain some information on the bulk of the generating function for the fugacities. Equation (4.20) suggests that the generating function for the fugacities takes the form

$$
\Theta(\xi, l)=\sum_{n=1}^{\infty} \frac{\xi^{n}}{n !} Y_{n}(l), \quad \xi \in \mathrm{C},
$$

with the initial condition

$$
\Theta(\xi, 0)=\frac{\varepsilon-i \eta}{\mathfrak{a}^{x_{1}-2}} \xi
$$

by Eq. (4.17). Differentiation with respect to the lattice rescaling parameter $l$ gives, with the help of Eq. (4.17), the second-order nonlinear partial differential equation

$$
\partial_{l} \Theta=\left[2-\xi \partial_{\xi}+\frac{g_{A}}{\pi}\left(\xi \partial_{\xi}\right)^{2}\right] \Theta+\pi \Theta^{2} .
$$

In turn, reparametrization of $\Theta$ and $\xi$

$$
\Theta(\xi, l)=: \frac{2}{\pi}[\widetilde{G}(y, l)-1], \quad \xi=:-e^{-y},
$$

gives

$$
\begin{gathered}
\partial_{l} \widetilde{G}=\left(\partial_{y}+\frac{g_{A}}{\pi} \partial_{y}^{2}\right) \widetilde{G}+2 \widetilde{G}(\widetilde{G}-1), \\
\widetilde{G}(y, 0)=1-\frac{\pi}{2} \frac{\varepsilon-i \eta}{\mathfrak{a}^{x_{1}-2}} e^{-y} .
\end{gathered}
$$

The convection term on the right-hand side is induced by the RG flow of the matter fields in the bosonic sector. This term is absent from the RG analysis of the normalization (4.18) of the zero mode. At the level of the generating function, the convection term can be disposed of by the change of variable consisting of a Galilean boost and of a rescaling

$$
G(x, t):=\widetilde{G}\left(\sqrt{g_{A} / \pi} x-t / 2, t / 2\right),
$$

after which

$$
\begin{gathered}
\partial_{t} G=\frac{1}{2} \partial_{x}^{2} G+G(G-1), \\
G(x, 0)=1-\frac{\pi}{2} \frac{\varepsilon-i \eta}{\mathfrak{a}^{x_{1}-2}} \exp \left(-\sqrt{\frac{g_{A}}{\pi}} x\right) \\
\approx \exp \left[-\frac{\pi}{2} \frac{\varepsilon-i \eta}{\mathfrak{a}^{x_{1}-2}} \exp \left(-\sqrt{\frac{g_{A}}{\pi}} x\right)\right] .
\end{gathered}
$$

The error caused by exponentiation in the last step should be comparable to the one caused by neglecting the renormalization of $g_{A}$ induced by the energy perturbation. Equation (4.23b) is known in the mathematical literature as the KPP equation with the forcing term

$$
F(G):=G(G-1)
$$

when $x \in \mathrm{R}$ and $0 \leqslant G \leqslant 1.51,52$

Trading $\Theta(\xi, l)$ for $\widetilde{G}(y, l)$ [or $G(x, t)$ ] offers the following advantage. It is possible to interpret $\widetilde{G}(y, l)$ as the generating function of a random variable $Z$ with the (scaledependent) probability distribution $P(Z, l)$ through the definition

$$
\widetilde{G}(y, l)=:\left\langle\exp \left(-e^{-y} Z\right)\right\rangle_{P(Z, l)}
$$

with the initial condition

$$
P(Z, 0)=\delta\left(Z-\pi Y_{1}(0) / 2\right) .
$$

This definition is consistent since the probability distribution $P(Z, l)$ is normalized to 1 initially and since its normalization is invariant under the RG flow. In turn, we can reinterpret the fugacities $Y_{n}$ as the moments of the (scaledependent) probability distribution $P(Z, l)$ with the initial conditions

$$
Y_{n}(l=0)=\frac{2}{\pi}\left(\frac{\pi}{2} \frac{\varepsilon-i \eta}{\mathfrak{a}^{x_{1}-2}}\right)^{n} .
$$

Equation (4.25c) should be compared with Eq. (4.17). This immediately leads to the identification of the (scaledependent) random variable $Z$ with the scale-dependent random energy $(\pi / 2)[\varepsilon(l)-i \eta(l)] / \mathfrak{a}^{x_{1}-2}$, i.e.,

$$
\begin{aligned}
\widetilde{G}(y, l) & =1+\frac{\pi}{2} \Theta(\xi, l) \\
& =:\left\langle\exp \left[-e^{-y} \frac{\pi}{2} \frac{\varepsilon(l)-i \eta(l)}{\mathfrak{a}^{x_{1}-2}}\right]\right\rangle_{P(Z, l)}
\end{aligned}
$$

and, more importantly, 


$$
\begin{aligned}
& \left\langle\ln \frac{Z(l)}{Z(0)}\right\rangle_{P(Z, l)}=\left\langle\ln \frac{\varepsilon(l)}{\varepsilon(0)}\right\rangle_{P(Z, l)}, \\
& \frac{\varepsilon_{\text {typ }}(l)}{\varepsilon_{\text {typ }}(0)}:=\exp \left[\left\langle\ln \frac{Z(l)}{Z(0)}\right\rangle_{P(Z, l)}\right] .
\end{aligned}
$$

As we shall see in the next two subsections, it is possible to extract from Eq. (4.26c) a scaling exponent $z_{A}$ called the dynamical scaling exponent that encodes the scaling of the typical energy $\varepsilon_{\text {typ }}(l)$ in the infrared limit $l \rightarrow \infty$ through

$$
z_{A}:=\lim _{l \rightarrow \infty} \frac{1}{l} \ln \frac{\varepsilon_{\mathrm{typ}}(l)}{\varepsilon_{\mathrm{typ}}(0)} .
$$

Knowledge of the dynamical scaling exponent $z_{A}$ suffices to compute the DOS in the MDH model.

\section{Analysis of the KPP equation}

In the limit $t \rightarrow \infty$, the KPP equation (4.23b) possesses a solution in the form of a traveling wave $w_{A}\left(x-v_{A} t\right)$ that is unique up to translation and whose velocity $v_{A}$ is solely controlled by the exponential decay of the initial data for large $x$, provided the initial data obey the conditions

$$
G(x, 0)= \begin{cases}1, & \text { for } x \rightarrow+\infty, \\ 0, & \text { for } x \rightarrow-\infty,\end{cases}
$$

which they do according to Eq. (4.23c). More precisely, if we choose the origin of $x$ such that

$$
\lim _{x \rightarrow \infty}[1-G(x, 0)]=e^{-\sqrt{g_{A} / \pi} x},
$$

then

$$
v_{A}= \begin{cases}\frac{1}{2} \sqrt{\frac{g_{A}}{\pi}}+\sqrt{\frac{\pi}{g_{A}}}, & \text { for } \frac{g_{A}}{\pi}<2 \\ \sqrt{2}, & \text { for } \frac{g_{A}}{\pi} \geqslant 2\end{cases}
$$

and

$$
\lim _{x \rightarrow \infty}\left[1-w_{A}(x)\right]= \begin{cases}e^{-\sqrt{g_{A} / \pi} x}, & \text { for } v_{A}>\sqrt{2}, \\ x e^{-\sqrt{2} x}, & \text { for } v_{A}=\sqrt{2}\end{cases}
$$

It is a remarkable property of the KPP equation ${ }^{51,52}$ that the form of the forcing term $F(G)$ in Eq. (4.24) does not affect the velocity of the traveling wave as long as

$$
\begin{gathered}
F(0)=F(1)=0, \quad F(G)<0, \\
F^{\prime}(0)=-1, \quad F^{\prime}(G) \geqslant-1, \quad 0<G<1 .
\end{gathered}
$$

As noted in Refs. 35 and 44, had we chosen a different RG scheme such as smoothened the hard-core condition in real space used to obtain Eq. (4.15), say, we would have obtained another forcing term in the KPP equation. Universality, i.e., the independence of the choice of $R G$ scheme, is thus equivalent to finding a forcing term that obeys Eq. (4.32) from the point of view of the KPP equation.
Equations (4.30) and (4.31) allow us to perform the summation over all fugacities in the generating function (4.23a) when $l \rightarrow \infty$ :

$$
\begin{aligned}
1-G(x, 2 l) & =-\frac{\pi}{2} \sum_{n=1}^{\infty} \frac{\left(-e^{-\sqrt{g_{A} / \pi} x+l}\right)^{n}}{n !} Y_{n}(l) \\
& = \begin{cases}e^{-\sqrt{g_{A} / \pi}\left(x-2 v_{A} l\right)}, & \text { for } v_{A}>\sqrt{2}, \\
x e^{-\sqrt{2}\left(x-2 v_{A} l\right)}, & \text { for } v_{A}=\sqrt{2},\end{cases}
\end{aligned}
$$

as $x \rightarrow \infty$. Although individual fugacities are relevant and grow without bounds in the infrared limit, a finite limiting value of the generating function can nevertheless be extracted if $x$ scales linearly with $l$ as a result of the travelingwave asymptotic of $G(x, 2 l)$.

\section{KPP equation and DOS}

We are ready to make an estimate for Eq. (4.26b) that justifies the definition of the dynamical exponent (4.27). To this end, we take advantage of the facts that, by Eqs. (4.23a), (4.23c), and (4.33), the generating function $G(x, 2 l)$ can be thought of as a wave front which, initially, was chosen to coincide with the origin $x=0$ and is located at $x=2 v_{A} l$ in the long-time limit $t=2 l \rightarrow \infty$. In the same way, $\widetilde{G}(y, l)$ $=G\left(\sqrt{\pi / g_{A}}(y+l), 2 l\right)$ is a wave front located at $y=0$ and $y=\left(2 \sqrt{g_{A} / \pi} v_{A}-1\right) l$ initially and in the infrared limit $l$ $\rightarrow \infty$, respectively. This gives the estimate

$$
\begin{aligned}
\left\langle\ln \frac{Z(l)}{Z(0)}\right\rangle_{P(Z, l)}= & \int_{-\infty}^{+\infty} d y[\widetilde{G}(y, 0)-\widetilde{G}(y, l)] \\
= & \sqrt{\frac{g_{A}}{\pi}} \int_{-\infty}^{+\infty} d x[G(x, 0) \\
& \left.-G\left(x+l \sqrt{\pi / g_{A}}, 2 l\right)\right] \\
= & \left(2 \sqrt{\frac{g_{A}}{\pi}} v_{A}-1\right) l+\mathcal{O}(\ln l),
\end{aligned}
$$

where the logarithmic corrections are universal (see Ref. 52). It is then tempting to identify

$$
\frac{\varepsilon_{\text {typ }}(l)}{\varepsilon_{\text {typ }}(0)}=\exp \left(\left\langle\ln \frac{Z(l)}{Z(0)}\right\rangle_{P(Z, l)}\right) \propto e^{z_{A} l},
$$

whereby

$$
z_{A}:=\lim _{l \rightarrow \infty} \frac{d \ln \varepsilon_{\mathrm{typ}}}{d l}= \begin{cases}1+\frac{g_{A}}{\pi}, & \text { for } g_{A}<2 \pi, \\ 4 \sqrt{\frac{g_{A}}{2 \pi}}-1, & \text { for } g_{A} \geqslant 2 \pi,\end{cases}
$$

defines the quenched average value $z_{A}$ of the dynamical exponent, as a coupling constant that enters the CallanSymanzik equation obeyed by the DOS, which we here assume to be self-averaging and to obey some sort of scaling 
ansatz. This conjecture is bolstered by the fact (see Refs. 45 and 46) that the distribution of $\ln Z(l)$ is self-averaging in the infrared limit $l \rightarrow \infty$ as we had hoped for. Consequently, it is plausible that $Y_{\text {typ }} \sim \varepsilon_{\text {typ }}$ is the only relevant coupling constant that enters the Callan-Symanzik equation for the (selfaveraging) DOS

$$
0=\left[z_{A} \varepsilon \frac{\partial}{\partial \varepsilon}-\left(2-z_{A}\right)\right] \nu(\varepsilon) .
$$

(Observe that $2-z_{A}=1-g_{A} / \pi=x_{1}$ for $g_{A}<2 \pi$.) A rigorous proof that the solutions to Eqs. (4.37) and (4.17) are identical in the thermodynamic limit $L \rightarrow \infty$ at some fixed energy $\varepsilon$ is beyond the scope of this paper.

The mechanism by which solutions to the KPP equations are traveling waves causes the dynamical exponent $z_{A}$ to undergo a freezing transition when the disorder strength $g_{A}$ reaches the critical value $2 \pi$. The freezing transition of the dynamical exponent reflects itself by a nonanalyticity of the multifractal spectrum of zero modes as was shown in Refs. 38 and 40. It also reveals itself through a nonanalytic dependence on $g_{A}$ in the power-law behavior of the DOS since integration of Eq. (4.37) yields

$$
\begin{aligned}
\frac{\nu(\varepsilon)}{\nu\left(\varepsilon_{\mathrm{ren}}\right)}= & \left(\frac{\varepsilon}{\varepsilon_{\mathrm{ren}}}\right)^{\left(2-z_{A}\right) / z_{A}} \\
& = \begin{cases}\left(\frac{\varepsilon}{\varepsilon_{\mathrm{ren}}}\right)^{\left(1-g_{A} / \pi\right) /\left(1+g_{A} / \pi\right)}, & \text { for } g_{A}<2 \pi, \\
\left(\frac{\varepsilon}{\varepsilon_{\mathrm{ren}}}\right)^{\left(3-4 \sqrt{g_{A} /(2 \pi)}\right) /\left(4 \sqrt{g_{A} /(2 \pi)}-1\right)},\end{cases}
\end{aligned}
$$

The energy scale $\varepsilon_{\text {ren }}$ is the one for which errors caused by neglecting renormalization effects on $g_{A}$ are of order 1. For weak disorder $\left(g_{A}<2 \pi\right)$, quenched and annealed average values of the dynamical exponent agree since $2-z_{A}=x_{1}$. For strong disorder $\left(g_{A} \geqslant 2 \pi\right)$, quenched and annealed average values of the dynamical exponent differ since $2-z_{A}$ $\neq x_{1}$. Bounds on the dynamical exponent that are consistent with Eq. (4.36) were established in Ref. 30. The same powerlaw dependence for the DOS of a single species of Dirac fermions subjected to a real random vector potential was derived in Ref. 9 for weak disorder. In this simpler context, the transition from weak to strong disorder for the DOS was established in Ref. 42 using a replica variational method. We now turn our attention to the computation of the DOS in the HWK model for which a field-theoretical approach is lacking.

\section{DOS FOR $\boldsymbol{H}_{\mathrm{HWK}}$}

The HWK model differs from the MDH model by the presence of a complex-valued random mass $M_{-}(\boldsymbol{r})$ $=\left[M_{+}(\boldsymbol{r})\right]^{*}$. It was pointed out in Ref. 32 that, although $g_{A}$ is exactly marginal in the MDH model (3.17), the presence of a random mass renders $g_{A}$ marginally relevant (in the sense that it grows logarithmically with length) whereas $g_{M}$ remains marginal in a one-loop RG analysis. Guruswamy et al. computed the exact $\beta$ functions for $g_{A}$ and $g_{M}$, thereby confirming the one-loop RG analysis. ${ }^{27}$ Furthermore, they found the anomalous scaling dimension

$$
\begin{gathered}
x_{\varepsilon}=\zeta\left[1+\mathcal{O}\left(g_{M}\right)\right]-\frac{g_{A}}{\pi} \zeta^{2}, \\
\zeta:=\frac{1}{1+g_{M} / \pi},
\end{gathered}
$$

for the energy perturbation in Eq. (3.15) by taking advantage of enhanced symmetries of the HWK model at the band center. Remarkably, the dependence on $g_{A}$ in Eq. (5.1) is exact while that on $g_{M}$ can be obtained by perturbation theory. Finally, they wrote down a Callan-Symanzik equation for the DOS of the form (4.6) with a finite number (3) of coupling constants. The three coupling constants are $\varepsilon, g_{A}$, and $g_{M}$. The corresponding $\beta$ functions are

$$
\beta_{\varepsilon}=\left(2-x_{\varepsilon}\right) \varepsilon, \quad \beta_{g_{A}}=\frac{\left(g_{M} \zeta\right)^{2}}{2 \pi^{2}}, \quad \beta_{g_{M}}=0
$$

respectively. Additionally, the anomalous scaling dimension $x_{\varepsilon}$ entering the Callan-Symanzik equation is given in Eq. (5.1). Solving this Callan-Symanzik equation gives the diverging DOS (1.1) in the vicinity of the band center.

One can tune the HWK model to the MDH model by switching off the random mass perturbation, i.e., by taking the limit $g_{M}=0$. In this limit, the Callan-Symanzik equation used by Guruswamy et al. only agrees with the CallanSymanzik equation (4.37) for the DOS when $g_{A}<2 \pi$, i.e., below the freezing transition. As we have seen in Sec. IV, this discrepancy is caused by a failure to account for all the relevant coupling constants associated with powers of the energy perturbation. In this section, we are going to show that infinitely many relevant operators were overlooked by Guruswamy et al. in their RG analysis of Eq. (3.15) at the band center. We will then derive a generalized KPP equation for the generating function of these coupling constants. This generalized KPP equation differs from the KPP equation (4.22) by a scale-dependent diffusion "constant" driven by the scale dependence of $g_{A}$. Within an adiabatic approximation, we shall extract from this generalized KPP equation a Callan-Symanzik equation for the DOS of the form (4.37) except for the presence of the running coupling constant $g_{A}$ whose $\beta$ function is given by Eq. (5.2). However small the bare value of $g_{A}$, it always flows to infinitely strong disorder. The freezing transition must then necessarily affect the DOS in the HWK model. The solution for the DOS in the HWK model is in fact given by Eq. (1.1) with the exponent $\kappa=2 / 3$ instead of $\kappa=1 / 2$. It is through the larger exponent $\kappa=2 / 3$ that the freezing transition reveals itself as was noted by Motrunich et al. ${ }^{30} \mathrm{~A}$ more detailed analysis of the CallanSymanzik equation shows that the asymptotic regime (1.1) with $\kappa=2 / 3$ can be preceded by crossover regimes charac- 
TABLE I. Abelian bosonization rules for $\mathrm{gl}(2 \mid 2)$ matter fields and currents: flavor-diagonal components of the currents. The scalar fields $\phi_{a}, \varphi_{a-}$, and $\varphi_{a+}$ are related by $\phi_{a}(z, \bar{z})=\varphi_{a-}(z)+\varphi_{a+}(\bar{z})$. The scalar fields $\phi_{a}^{\prime}, \varphi_{a-}^{\prime}$, and $\varphi_{a+}^{\prime}$ are related by $\phi_{a}^{\prime}(z, \bar{z})=\varphi_{a-}^{\prime}(z)+\varphi_{a+}^{\prime}(\bar{z})$. The lattice spacing $\mathfrak{a}$ is used as a short-distance cutoff. The magnitude of the complex number $u$ is arbitrary as it reflects the nonuniversality of the short-distance regularization. The phase of the complex number $u$ is fixed by the boundary condition obeyed by the scalar fields at infinity, which, in turn, is uniquely fixed by the SUSY of the theory. Demanding that the scalar fields vanish at infinity amounts to fixing the phase of $u$ to be $-\pi / 2$. We will choose $u=$ $-i$ for convenience.

\begin{tabular}{lccc}
\hline \hline SUSY matter & Bosonized matter & SUSY currents & Bosonized currents \\
\hline & $u^{+1 / 2} \mathfrak{a}^{-1 / 2} e^{-i \varphi_{a-}}$ & $J_{a a}:=\psi_{a-} \psi_{a-}^{*}$ & $\left(+i \partial \phi_{a}\right)$ \\
$\psi_{a-}^{*}$ & $u^{-1 / 2} \mathfrak{a}^{-1 / 2} e^{+i \varphi_{a-}}$ & $\bar{J}_{a a}:=\psi_{a+} \psi_{a+}^{*}$ & $\left(-i \bar{\partial} \phi_{a}\right)$ \\
$\psi_{a-}$ & $\left(u^{*}\right)^{+1 / 2} \mathfrak{a}^{-1 / 2} e^{+i \varphi_{a+}}$ & $J_{a a}^{\prime}:=\beta_{a-} \beta_{a-}^{*}$ & $\left(+i \partial \phi_{a}^{\prime}\right)$ \\
$\psi_{a+}^{*}$ & $\left(u^{*}\right)^{-1 / 2} \mathfrak{a}^{-1 / 2} e^{-i \varphi_{a+}}$ & $\bar{J}_{a a}^{\prime}:=\beta_{a+} \beta_{a+}^{*}$ & $\left(-i \bar{\partial} \phi_{a}^{\prime}\right)$ \\
$\psi_{a+}$ & $u^{-1 / 2} \mathfrak{a}^{+1 / 2} e^{-i \varphi_{a-}^{\prime}\left(i \partial \chi_{a}\right)}$ & $G_{a a+}:=\beta_{a-}^{*} \psi_{a-}$ & $u^{-1} e^{-i\left(\varphi_{a-}^{\prime} \varphi_{a-}\right)}\left(i \partial \chi_{a}\right)$ \\
$\beta_{a-}^{*}$ & $u^{+1 / 2} \mathfrak{a}^{+1 / 2} e^{+i \varphi_{a-}^{\prime}\left(i \partial \chi_{a}^{*}\right)}$ & $\bar{G}_{a a+}:=\beta_{a+}^{*} \psi_{a+}$ & $\left(u^{*}\right)^{-1} e^{+i\left(\varphi_{a+}^{\prime} \varphi_{a+}\right)}\left(i \bar{\partial} \chi_{a}\right)$ \\
$\beta_{a-}$ & $\left(u^{*}\right)^{-1 / 2} \mathfrak{a}^{+1 / 2} e^{+i \varphi_{a+}^{\prime}\left(i \bar{\partial} \chi_{a}\right)}$ & $G_{a a-}:=\beta_{a-} \psi_{a-}^{*}$ & $u e^{+i\left(\varphi_{a-}^{\prime} \varphi_{a-}\right)}\left(i \partial \chi_{a}^{*}\right)$ \\
$\beta_{a+}^{*}$ & $\left(u^{*}\right)^{+1 / 2} \mathfrak{a}^{+1 / 2} e^{-i \varphi_{a+}^{\prime}\left(i \bar{\partial} \chi_{a}^{*}\right)}$ & $\bar{G}_{a a-}:=\beta_{a+} \psi_{a+}^{*}$ & $u^{*} e^{-i\left(\varphi_{a+}^{\prime} \varphi_{a+}\right)}\left(i \bar{\partial} \chi_{a}^{*}\right)$ \\
$\beta_{a+}$ & & & \\
\hline \hline
\end{tabular}

terized by the Gade singularity or by the power-law behavior of the MDH model provided bare coupling constants are fine-tuned.

\section{A. Nearly conformal field theory}

The chiral decoupling transformation (3.14) on the HWK model in the SUSY representation (3.13) is not as useful when $g_{M}>0$ as it is when $g_{M}=0$ since rotated SUSY matter fields remain coupled to the disorder through the random mass. Our starting point in this section will thus be the effective theory obtained from the HWK model at the band center after integration over the Gaussian-distributed random vector and mass potentials

$$
\begin{aligned}
& Z:=\int \mathcal{D}\left[\psi^{*}, \psi, \beta^{*}, \beta\right] e^{-\int\left(d^{2} r / \pi\right)\left[\mathcal{L}_{0}+\left(g_{A} / 2 \pi\right) \mathcal{O}_{A}+\left(g_{M} / 2 \pi\right) \mathcal{O}_{M}\right],}, \\
& \mathcal{L}_{0}:=\sum_{a=1}^{2}\left(\psi_{a-}^{*} \bar{\partial} \psi_{a-}+\psi_{a+}^{*} \partial \psi_{a+}+\beta_{a-}^{*} \bar{\partial} \beta_{a-}+\beta_{a+}^{*} \partial \beta_{a+}\right), \\
& \mathcal{O}_{A}=-\sum_{a, b=1}^{2}\left(\psi_{a-}^{*} \psi_{a-}+\beta_{a-}^{*} \beta_{a-}\right)\left(\psi_{b+}^{*} \psi_{b+}+\beta_{b+}^{*} \beta_{b+}\right), \\
& \mathcal{O}_{M}=-\sum_{a, b=1}^{2}\left(\psi_{a+}^{*} \psi_{a-}+\beta_{a+}^{*} \beta_{a-}\right)\left(\psi_{b-}^{*} \psi_{b+}+\beta_{b-}^{*} \beta_{b+}\right) .
\end{aligned}
$$

TABLE II. Abelian bosonization rules for $\mathrm{gl}(2 \mid 2)$ matter fields and currents: flavor-off-diagonal components of the currents, i.e., $a \neq b$. The scalar fields $\phi_{a}, \varphi_{a-}$, and $\varphi_{a+}$ are related by $\phi_{a}(z, \bar{z})=\varphi_{a-}(z)$ $+\varphi_{a+}(\bar{z})$. The scalar fields $\phi_{a}^{\prime}, \varphi_{a-}^{\prime}$, and $\varphi_{a+}^{\prime}$ are related by $\phi_{a}^{\prime}(z, \bar{z})=\varphi_{a-}^{\prime}(z)+\varphi_{a+}^{\prime}(\bar{z})$. The lattice spacing $\mathfrak{a}$ is used as a short-distance cutoff. The magnitude of the complex number $u$ is arbitrary as it reflects the nonuniversality of the short-distance regularization. The phase of the complex number $u$ is fixed by the

\begin{tabular}{|c|c|c|c|}
\hline SUSY matter & Bosonized matter & SUSY currents & Bosonized currents \\
\hline$\psi_{a-}^{*}$ & $u^{+1 / 2} \mathfrak{a}^{-1 / 2} e^{-i \varphi_{a-}}$ & $J_{b a}:=\psi_{a-} \psi_{b-}^{*}$ & $\mathfrak{a}^{-1} e^{+i\left(\varphi_{a-}-\varphi_{b-}\right)}$ \\
\hline$\psi_{a-}$ & $u^{-1 / 2} \mathfrak{a}^{-1 / 2} e^{+i \varphi_{a-}}$ & $\bar{J}_{b a}:=\psi_{a+} \psi_{b+}^{*}$ & $\mathfrak{a}^{-1} e^{-i\left(\varphi_{a+}-\varphi_{b+}\right)}$ \\
\hline$\psi_{a+}^{*}$ & $\left(u^{*}\right)^{+1 / 2} \mathfrak{a}^{-1 / 2} e^{+i \varphi_{a+}}$ & $J_{b a}^{\prime}:=\beta_{a-} \beta_{b-}^{*}$ & $\mathfrak{a} e^{+i\left(\varphi_{a-}^{\prime}-\varphi_{b-}^{\prime}\right)}\left(i \partial \chi_{a}^{*}\right)\left(i \partial \chi_{b}\right)$ \\
\hline$\psi_{a+}$ & $\left(u^{*}\right)^{-1 / 2} \mathfrak{a}^{-1 / 2} e^{-i \varphi_{a+}}$ & $\bar{J}_{b a}^{\prime}:=\beta_{a+} \beta_{b+}^{*}$ & $\mathfrak{a} e^{-i\left(\varphi_{a+}^{\prime}-\varphi_{b+}^{\prime}\right)}\left(i \bar{\partial} \chi_{a}^{*}\right)\left(i \bar{\partial} \chi_{b}\right)$ \\
\hline$\beta_{a-}^{*}$ & $u^{-1 / 2} \mathfrak{a}^{+1 / 2} e^{-i \varphi_{a-}^{\prime}\left(i \partial \chi_{a}\right)}$ & $G_{b a+}:=\beta_{a-}^{*} \psi_{b-}$ & $u^{-1} e^{-i\left(\varphi_{a-}^{\prime}-\varphi_{b-}\right)}\left(i \partial \chi_{a}\right)$ \\
\hline$\beta_{a-}$ & $u^{+1 / 2} \mathfrak{a}^{+1 / 2} e^{+i \varphi_{a-}^{\prime}\left(i \partial \chi_{a}^{*}\right)}$ & $\bar{G}_{b a+}:=\beta_{a+}^{*} \psi_{b+}$ & $\left(u^{*}\right)^{-1} e^{+i\left(\varphi_{a+}^{\prime}-\varphi_{b+}\right)}\left(i \bar{\partial} \chi_{a}\right)$ \\
\hline$\beta_{a+}^{*}$ & $\left(u^{*}\right)^{-1 / 2} \mathfrak{a}^{+1 / 2} e^{+i \varphi_{a+}^{\prime}\left(i \bar{\partial} \chi_{a}\right)}$ & $G_{b a-}:=\beta_{a-} \psi_{b-}^{*}$ & $u e^{+i\left(\varphi_{a-}^{\prime}-\varphi_{b-}\right)}\left(i \partial \chi_{a}^{*}\right)$ \\
\hline$\beta_{a+}$ & $\left(u^{*}\right)^{+1 / 2} \mathfrak{a}^{+1 / 2} e^{-i \varphi_{a+}^{\prime}}\left(i \bar{\partial} \chi_{a}^{*}\right)$ & $\bar{G}_{b a-}:=\beta_{a+} \psi_{b+}^{*}$ & $u^{*} e^{-i\left(\varphi_{a+}^{\prime}-\varphi_{b+}\right)}\left(i \bar{\partial} \chi_{a}^{*}\right)$ \\
\hline
\end{tabular}
boundary condition obeyed by the scalar fields at infinity, which, in turn, is uniquely fixed by the SUSY of the theory. Demanding that the scalar fields vanish at infinity amounts to fixing the phase of $u$ to be $-\pi / 2$. We will choose $u=-i$ for convenience. 
TABLE III. Abelian bosonization rules for mass and vector potential current-current interactions: flavordiagonal contributions. Columns 1 and 2 are the contributions to the mass perturbation and columns 3 and 4 are the contributions to the vector perturbation. With the choice $u=-i$ in Tables I and II $u^{-1} u^{*}$ $=u\left(u^{*}\right)^{-1}=-1$.

\begin{tabular}{lccc}
\hline \hline SUSY matter & Bosonized matter & SUSY matter & Bosonized matter \\
\hline$+J_{a a} \bar{J}_{a a}$ & $+\left(\partial \phi_{a}\right)\left(\bar{\partial} \phi_{a}\right)$ & $-J_{a a} \bar{J}_{a a}$ & $-\left(\partial \phi_{a}\right)\left(\bar{\partial} \phi_{a}\right)$ \\
$-J_{a a}^{\prime} \bar{J}_{a a}^{\prime}$ & $-\left(\partial \phi_{a}^{\prime}\right)\left(\bar{\partial} \phi_{a}^{\prime}\right)$ & $-J_{a a}^{\prime} \bar{J}_{a a}^{\prime}$ & $-\left(\partial \phi_{a}^{\prime}\right)\left(\bar{\partial} \phi_{a}^{\prime}\right)$ \\
$+G_{a a+} \bar{G}_{a a-}$ & $+u^{-1} u^{*} e^{-i\left(\phi_{a}^{\prime}-\phi_{a}\right)}\left(i \partial \chi_{a}\right)\left(i \bar{\partial} \chi_{a}^{*}\right)$ & $+J_{a a} \bar{J}_{a a}^{\prime}$ & $+\left(\partial \phi_{a}\right)\left(\bar{\partial} \phi_{a}^{\prime}\right)$ \\
$-G_{a a-} \bar{G}_{a a+}$ & $-u\left(u^{*}\right)^{-1} e^{+i\left(\phi_{a}^{\prime}-\phi_{a}\right)}\left(i \partial \chi_{a}^{*}\right)\left(i \bar{\partial} \chi_{a}\right)$ & $+J_{a a}^{\prime} \bar{J}_{a a}$ & $+\left(\partial \phi_{a}^{\prime}\right)\left(\bar{\partial} \phi_{a}\right)$ \\
\hline \hline
\end{tabular}

Here $\bar{\partial}:=\frac{1}{2} \partial_{+}$and $\partial:=\frac{1}{2} \partial_{-}$. Observe that the effective theory (5.3) is invariant under any permutation of the flavor indices. Flavor permutation symmetry is broken by the energy perturbation (3.13c); i.e., the SUSY symmetry group in the presence of an energy perturbation is enlarged to the group $\mathrm{GL}(2 \mid 2)$ at the band center. Since we are after the behavior of the DOS in the immediate vicinity of the band center, it is essential to account for the enlarged SUSY symmetry group $\operatorname{GL}(2 \mid 2)$ at the band center.

Fermionic interacting field theories can be considerably simplified by the method of bosonization. This is also the case of the SUSY representation (5.3) of the HWK model at the band center as shown by Guruswamy et al., ${ }^{27}$ and we now summarize their results. We thus adopt Abelian bosonization rules that are summarized in Tables I-IV. ${ }^{53}$ Abelian bosonization in the fermionic sector is performed by trading left, $\psi_{a-}^{*}$, say, and right, $\psi_{a+}^{*}$, say, moving fermions for the exponential of holomorphic $\varphi_{a-}$ and antiholomorphic $\varphi_{a+}$ scalar fields, respectively. Abelian bosonization in the bosonic sector was introduced in string theory. ${ }^{54,55}$ It is performed by trading left, $\beta_{a-}^{*}$, say, and right, $\beta_{a+}^{*}$, say, moving bosons for the exponential of holomorphic $\varphi_{a-}^{\prime}$ and antiholomorphic $\varphi_{a+}^{\prime}$, scalar fields, respectively. Additional fermionic fields $\chi_{a}^{*}$ and $\chi_{a}$ must also be introduced when bosonizing bosonic spinors. A simplifying feature of the two interactions induced by the disorder in Eq. (5.3) is that they can be written as bilinears in the $\mathrm{gl}(2 \mid 2)$ currents that generate the GL(2|2) symmetry group. These currents are of two kinds. The first kind is made of bosonic currents denoted $J_{a b}, \bar{J}_{a b}, J_{a b}^{\prime}$, and $\bar{J}_{a b}^{\prime}$, which are defined in Tables I and II. The second kind is made of fermionic currents denoted $G_{a b+}, \bar{G}_{a b+}, G_{a b-}$, and $\bar{G}_{a b-}$, which are also defined in Tables I and II. Currents of the bosonic kind permute the flavors of right and left movers but do not change their statistics. Currents of the fermionic kind exchange fermionic spinors of one flavor for bosonic spinors of another flavor, and conversely. The importance of the $\operatorname{gl}(N \mid N), N \in \mathbb{N}$ algebra for Dirac fermions coupled to random fields was emphasized in Refs. 36, 37, and 43. The central role of the symmetry group $\operatorname{GL}(N \mid N)$ also appears in the quantum field theory description of the multivariable Alexander polynomial. ${ }^{56}$

After Abelian bosonization, the holomorphic and antiholomorphic scalar fields $\varphi_{a \pm}$ and $\varphi_{a \pm}^{\prime}$ enter Eq. (5.3) through the combinations

$$
\phi_{a}:=\varphi_{a-}+\varphi_{a+}, \quad \phi_{a}^{\prime}:=\varphi_{a-}^{\prime}+\varphi_{a+}^{\prime}, \quad a=1,2
$$

only (see Tables III and IV),

TABLE IV. Abelian bosonization rules for mass and vector potential current-current interactions: flavoroff-diagonal contributions, i.e., $a \neq b$. The lattice spacing $\mathfrak{a}$ is used as a short-distance cutoff. Columns 1 and 2 are the contributions to the mass perturbation, and columns 3 and 4 are the contributions to the vector perturbation. With the choice $u=-i$ in Tables I and II $u^{-1} u^{*}=u\left(u^{*}\right)^{-1}=-1$.

\begin{tabular}{lccc}
\hline \hline SUSY matter & Bosonized matter & SUSY matter & Bosonized matter \\
\hline$+J_{b a} \bar{J}_{a b}$ & $+\mathfrak{a}^{-2} e^{+i\left(\phi_{a}-\phi_{b}\right)}$ & $-J_{a a} \bar{J}_{b b}$ & $-\left(\partial \phi_{a}\right)\left(\bar{\partial} \phi_{b}\right)$ \\
$-J_{b a}^{\prime} \bar{J}_{a b}^{\prime}$ & $-\mathfrak{a}^{+2} e^{+i\left(\phi_{a}^{\prime}-\phi_{b}^{\prime}\right)}\left(\partial \chi_{a}^{*}\right)\left(\partial \chi_{b}\right)\left(\bar{\partial} \chi_{b}^{*}\right)\left(\bar{\partial} \chi_{a}\right)$ & $-J_{a a}^{\prime} \bar{J}_{b b}^{\prime}$ & $-\left(\partial \phi_{a}^{\prime}\right)\left(\bar{\partial} \phi_{b}^{\prime}\right)$ \\
$+G_{a b+} \bar{G}_{a b-}$ & $+u^{-1} u^{*} e^{-i\left(\phi_{b}^{\prime}-\phi_{a}\right)}\left(i \partial \chi_{b}\right)\left(i \bar{\partial} \chi_{b}^{*}\right)$ & $+J_{a a} \bar{J}_{b b}^{\prime}$ & $+\left(\partial \phi_{a}\right)\left(\bar{\partial} \phi_{b}^{\prime}\right)$ \\
$-G_{b a-} \bar{G}_{b a+}$ & $-u\left(u^{*}\right)^{-1} e^{+i\left(\phi_{a}^{\prime}-\phi_{b}\right)}\left(i \partial \chi_{a}^{*}\right)\left(i \bar{\partial} \chi_{a}\right)$ & $+J_{a a}^{\prime} \bar{J}_{b b}$ & $+\left(\partial \phi_{a}^{\prime}\right)\left(\bar{\partial} \phi_{b}\right)$ \\
\hline \hline
\end{tabular}




$$
\begin{aligned}
& Z=\int \mathcal{D}\left[\phi_{a}, \phi_{a}^{\prime}, \chi_{a}^{*}, \chi_{a}\right] \exp \left(-\int \frac{d^{2} \boldsymbol{r}}{2 \pi} \mathcal{L}\right), \quad \mathcal{L}:=\mathcal{L}_{1}+\mathcal{L}_{2}, \\
& \mathcal{L}_{1}=\left(1+\frac{g_{M}}{\pi}\right) \sum_{a=1}^{2}\left[\left(\partial \phi_{a}\right)\left(\bar{\partial} \phi_{a}\right)-\left(\partial \phi_{a}^{\prime}\right)\left(\bar{\partial} \phi_{a}^{\prime}\right)\right]-\frac{g_{A}}{\pi}\left(\partial \Sigma_{-}\right)\left(\bar{\partial} \Sigma_{-}\right) \\
& -\sum_{a=1}^{2}\left\{\left[1+\frac{g_{M}}{\pi} e^{-i\left(\phi_{a}-\phi_{a}^{\prime}\right)}\right]\left(\partial \chi_{a}^{*}\right)\left(\bar{\partial} \chi_{a}\right)+\left[1+\frac{g_{M}}{\pi} e^{+i\left(\phi_{a}-\phi_{a}^{\prime}\right)}\right]\left(\bar{\partial} \chi_{a}^{*}\right)\left(\partial \chi_{a}\right)\right\}, \\
& \mathcal{L}_{2}=\frac{g_{M}}{\pi} \sum_{a \neq b}^{2}\left[\frac{1}{\mathfrak{a}^{2}} e^{+i\left(\phi_{a}-\phi_{b}\right)}-\mathfrak{a}^{2} e^{+i\left(\phi_{a}^{\prime}-\phi_{b}^{\prime}\right)}\left(\partial \chi_{a}^{*}\right)\left(\partial \chi_{b}\right)\left(\bar{\partial} \chi_{b}^{*}\right)\left(\bar{\partial} \chi_{a}\right)-e^{-i\left(\phi_{b}-\phi_{a}^{\prime}\right)}\left(\partial \chi_{a}^{*}\right)\left(\bar{\partial} \chi_{a}\right)-e^{+i\left(\phi_{a}-\phi_{b}^{\prime}\right)}\left(\bar{\partial} \chi_{b}^{*}\right)\left(\partial \chi_{b}\right)\right]
\end{aligned}
$$

Here we have introduced the "center of mass" $\Sigma_{+}$and the "relative coordinate" $\Sigma_{-}$for the scalar fields

$$
\begin{gathered}
\Sigma_{+}:=\Sigma+\Sigma^{\prime}, \quad \Sigma_{-}:=\Sigma-\Sigma^{\prime}, \quad \Sigma:=\sum_{a=1}^{2} \phi_{a}, \\
\Sigma^{\prime}:=\sum_{a=1}^{2} \phi_{a}^{\prime} .
\end{gathered}
$$

After Abelian bosonization, the decoupling transformation (3.14) takes the very simple and useful form

$$
\begin{aligned}
& \mathcal{L}\left[\phi_{a}, \phi_{a}^{\prime}, \chi_{a}^{*}, \chi_{a} ; g_{A}, g_{M}\right] \\
& \quad=\mathcal{L}\left[\phi_{a}-\frac{g_{A}}{2 \pi} \zeta \Sigma_{-}, \phi_{a}^{\prime}-\frac{g_{A}}{2 \pi} \zeta \Sigma_{-}, \chi_{a}^{*}, \chi_{a} ; g_{A}=0, g_{M}\right] .
\end{aligned}
$$

As pointed out by Guruswamy et al., ${ }^{27} \mathrm{Eq}$. (5.7) is crucial to establish that the HWK model is, in essence, a nearly conformal field theory at the band center. Equation (5.7) follows from the peculiarity of the Lagrangian $\mathcal{L}$ by which the center of mass $\Sigma_{+}$only enters (in $\mathcal{L}_{1}$ ) through the linear term

$$
\frac{1}{2 \zeta}\left(\partial \Sigma_{+}\right)\left(\bar{\partial} \Sigma_{-}\right)
$$

To put it differently, the term (5.8) is the only one in $\mathcal{L}$ affected by the chiral transformation

$$
\begin{gathered}
\phi_{a} \rightarrow \phi_{a}+\frac{g_{A}}{2 \pi} \zeta \Sigma_{-}, \\
\phi_{a}^{\prime} \rightarrow \phi_{a}^{\prime}+\frac{g_{A}}{2 \pi} \zeta \Sigma_{-}, \quad a=1,2 .
\end{gathered}
$$

All other terms in $\mathcal{L}$ and, in particular, all terms in $\mathcal{L}_{2}$ are left unchanged by the chiral transformation (5.9) as is best seen after trading in $\mathcal{L}$ the four coordinates $\phi_{a}$ and $\phi_{a}^{\prime}(a=1,2)$ for $\Sigma_{+}, \Sigma_{-}$and the relative coordinates $\sigma_{a+}:=\sigma_{a}+\sigma_{a}^{\prime}$ and $\sigma_{a-}^{\prime}:=\sigma_{a}-\sigma_{a}^{\prime}$ whereby $\phi_{a}=:(\Sigma / 2)+\sigma_{a}$ and $\phi_{a}^{\prime}=:\left(\Sigma^{\prime} / 2\right)$ $+\sigma_{a}^{\prime}$. The role of the center of mass $\Sigma_{+}$is thus to enforce a global constraint on the coordinates $\phi_{a}$ and $\phi_{a}^{\prime}, a=1,2$ of the $\mathrm{gl}(2 \mid 2)$-graded Lie algebra.

We are mostly (if not only) concerned with the evaluation of the expectation value of the product of vertex operators (up to Klein factors that do not matter for the calculation of anomalous scaling dimensions)

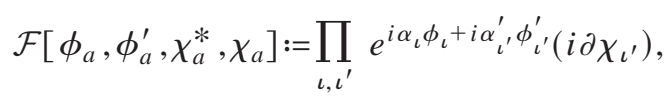

where $\iota$ and $\iota^{\prime}$ are collective labels for flavor and space coordinates, $\left(i \partial \chi_{\iota^{\prime}}\right)$ should be understood as any of the four options $\left(i \partial \chi_{\iota^{\prime}}\right),\left(i \bar{\partial} \chi_{\iota^{\prime}}\right),\left(i \partial \chi_{\iota^{\prime}}^{*}\right)$, and $\left(i \bar{\partial} \chi_{\iota^{\prime}}^{*}\right)$, and $\alpha_{\iota}, \alpha_{\iota^{\prime}}^{\prime}$ $\in \mathbb{R}$. Under the chiral (additive) transformation law (5.9) the transformation law of a product of vertex operators is multiplicative

$$
\begin{aligned}
\mathcal{F}[ & \left.\phi_{a}, \phi_{a}^{\prime}, \chi_{a}^{*}, \chi_{a}\right] \\
& \rightarrow e^{i\left(g_{A} / 2 \pi\right) \zeta \Sigma_{-}\left(\Sigma_{\iota} \alpha_{\iota}+\Sigma_{\iota^{\prime}} \alpha_{\iota^{\prime}}^{\prime}\right)} \mathcal{F}\left[\phi_{a}, \phi_{a}^{\prime}, \chi_{a}^{*}, \chi_{a}\right] .
\end{aligned}
$$

The usefulness of Eqs. (5.7), (5.9), and (5.11) is the following. If the expectation value of the left-hand side of Eq. (5.11) is to be evaluated with the partition function (5.3) at given $g_{M}$ and $g_{A}$, then the expectation value of the righthand side of Eq. (5.11) is to be evaluated at the same $g_{M}$ but different $g_{A}=0$. Let us denote by $\langle(\cdots)\rangle_{\mathcal{L}, g_{M}, g_{A}}$ the expectation value with the partition function (5.3). We have just proved that

$$
\begin{aligned}
\langle\mathcal{F}[ & \left.\left.\phi_{a}, \phi_{a}^{\prime}, \chi_{a}^{*}, \chi_{a}\right]\right\rangle_{\mathcal{L}, g_{M}, g_{A}} \\
& =\left\langle e^{i\left(g_{A} / 2 \pi\right) \zeta \Sigma_{-}\left(\Sigma_{\iota} \alpha_{\iota}+\Sigma_{\iota^{\prime}} \alpha_{\iota^{\prime}}^{\prime}\right)} \mathcal{F}\left[\phi_{a}, \phi_{a}^{\prime}, \chi_{a}^{*}, \chi_{a}\right]\right\rangle_{\mathcal{L}_{, g_{M}, 0}} .
\end{aligned}
$$

Let us denote by $\mathcal{G}\left[\phi_{a}, \phi_{a}^{\prime}, \chi_{a}^{*}, \chi_{a}\right]$ the right-hand side of Eq. (5.11). We also write $\langle(\cdots)\rangle_{\mathcal{L}_{1}, g_{M}, 0}$ for the expectation value with $\mathcal{L}$ in the partition function $(5.5)$ replaced by $\mathcal{L}_{1}$ with $g_{A}=0$. We then define the perturbative expansion of the 
expectation value $\left\langle\mathcal{G}\left[\phi_{a}, \phi_{a}^{\prime}, \chi_{a}^{*}, \chi_{a}\right]\right\rangle_{\mathcal{L}, g_{M}, 0}$ around the "unperturbed theory" $\mathcal{L}_{1}$ with $g_{A}=0$ by

$$
\begin{aligned}
\left\langle\mathcal{G}\left[\phi_{a}, \phi_{a}^{\prime}, \chi_{a}^{*}, \chi_{a}\right]\right\rangle_{\mathcal{L}, g_{M}, 0}=\sum_{n=0}^{\infty} \frac{(-1)^{n}}{n !} \mathcal{G}_{\mathcal{L}_{1}, g_{M}, 0}^{(n)}, \\
\mathcal{G}_{\mathcal{L}_{1}, g_{M}, 0}^{(n)}=\int \frac{d^{2} \boldsymbol{r}_{1}}{2 \pi} \cdots \int \frac{d^{2} \boldsymbol{r}_{n}}{2 \pi}\left\langle\mathcal{G}\left[\phi_{a}, \phi_{a}^{\prime}, \chi_{a}^{*}, \chi_{a}\right]\right. \\
\left.\quad \times \mathcal{L}_{2}\left(\boldsymbol{r}_{1}\right) \cdots \mathcal{L}_{2}\left(\boldsymbol{r}_{n}\right)\right\rangle_{\mathcal{L}_{1}, g_{M}, 0} .
\end{aligned}
$$

To go from Eq. (5.12) to Eq. (5.13) we made use of the fact that the perturbation $\mathcal{L}_{2}$ does not couple to the center of mass $\Sigma_{+}$and is thus invariant under the chiral transformation (5.9). Nearly conformal invariance is a property of the theory defined by Lagrangian $\mathcal{L}_{1}$ with $g_{A}=0$ as $\mathcal{L}_{1}$ then reduces to two independent copies of the $N=1$ limiting theory for which nearly conformal invariance was demonstrated in Ref. 27. Before we characterize in more detail what is meant by nearly conformal invariance, it suffices here to say that any perturbative contribution to the right-hand side of Eq. (5.12) is to be evaluated with the following rules. (i) All fields obey Wick's theorem. (ii) Any two-point function between $\Sigma_{-}$and the relative coordinates entering $\mathcal{L}_{2}$ vanishes. If so, perturbation theory in powers of $\mathcal{L}_{2}$ does not renormalize the dependence on $g_{A}$ of the expectation value of the right-hand side of Eq. (5.13) obtained to zeroth order in powers of $\mathcal{L}_{2}$. We have thus proved that the exact dependence on $g_{A}$ of any functional of the form (5.10) is already obtained to zeroth order in perturbation theory in powers of $\mathcal{L}_{2}$ around the unperturbed Lagrangian $\mathcal{L}_{1}$ with $g_{A}=0$ once the chiral transformation (5.11) has been performed.

Under nearly conformal invariance of the theory defined by the Lagrangian $\mathcal{L}_{1}$ with $g_{A}=0$, we understand the following properties demonstrated in Ref. 27.

(i) Nonvanishing two-point functions between the fields $\phi_{a}, \phi_{a}^{\prime}$ [see Eq. (5.4)], their duals

$$
\widetilde{\phi}_{a}:=\varphi_{a-}-\varphi_{a+}, \quad \widetilde{\phi}_{a}^{\prime}:=\varphi_{a-}^{\prime}-\varphi_{a+}^{\prime},
$$

and the auxiliary fermions $\chi_{a}^{*}$ and $\chi_{a} \operatorname{are}^{53}$

$$
\begin{aligned}
& \left\langle\phi_{a}(z, \bar{z}) \phi_{b}(0,0)\right\rangle_{\mathcal{L}, g_{M}, g_{A}} \\
& =\zeta\left[-\delta_{a b}-\left(g_{A} \zeta / \pi\right)\right] \ln \left(\frac{z \bar{z}}{\mathfrak{a}^{2}}\right) \\
& -\left[g_{M} \zeta /(2 \pi)\right]^{2} \zeta^{2} \ln ^{2}\left(\frac{z \bar{z}}{\mathfrak{a}^{2}}\right)+\cdots, \\
& \left\langle\phi_{a}^{\prime}(z, \bar{z}) \phi_{b}^{\prime}(0,0)\right\rangle_{\mathcal{L}, g_{M}, g_{A}} \\
& =\zeta\left[+\delta_{a b}-\left(g_{A} \zeta / \pi\right)\right] \ln \left(\frac{z \bar{z}}{\mathfrak{a}^{2}}\right) \\
& -\left[g_{M} \zeta /(2 \pi)\right]^{2} \zeta^{2} \ln ^{2}\left(\frac{z \bar{z}}{\mathfrak{a}^{2}}\right)+\cdots,
\end{aligned}
$$

$$
\begin{gathered}
\left\langle\phi_{a}(z, \bar{z}) \phi_{b}^{\prime}(0,0)\right\rangle_{\mathcal{L}, g_{M}, g_{A}} \\
=-\left(g_{A} \zeta^{2} / \pi\right) \ln \left(\frac{z \bar{z}}{\mathfrak{a}^{2}}\right)-\left[g_{M} \zeta /(2 \pi)\right]^{2} \zeta^{2} \ln ^{2}\left(\frac{z \bar{z}}{\mathfrak{a}^{2}}\right)+\cdots \\
\left\langle\widetilde{\phi}_{a}(z, \bar{z}) \widetilde{\phi}_{b}(0,0)\right\rangle_{\mathcal{L}, g_{M}, g_{A}} \\
=\zeta\left[-\delta_{a b}+\left(g_{A} \zeta / \pi\right)\right] \ln \left(\frac{z \bar{z}}{\mathfrak{a}^{2}}\right) \\
+\left[g_{M} \zeta /(2 \pi)\right]^{2} \zeta^{2} \ln \left(\frac{z \bar{z}}{\mathfrak{a}^{2}}\right)+\cdots \\
\left\langle\widetilde{\phi}_{a}^{\prime}(z, \bar{z}) \widetilde{\phi}_{b}^{\prime}(0,0)\right\rangle_{\mathcal{L}, g_{M}, g_{A}} \\
=\zeta\left[+\delta_{a b}+\left(g_{A} \zeta / \pi\right)\right] \ln \left(\frac{z \bar{z}}{\mathfrak{a}^{2}}\right) \\
+\left[g_{M} \zeta /(2 \pi)\right]^{2} \zeta^{2} \ln ^{2}\left(\frac{z \bar{z}}{\mathfrak{a}^{2}}\right)+\cdots \\
\left\langle\widetilde{\phi}_{a}(z, \bar{z}) \widetilde{\phi}_{b}^{\prime}(0,0)\right\rangle_{\mathcal{L}, g_{M}, g_{A}} \\
=+\left(g_{A} \zeta^{2} / \pi\right) \ln \left(\frac{z \bar{z}}{\mathfrak{a}^{2}}\right)+\left[g_{M} \zeta /(2 \pi)\right]^{2} \zeta^{2} \ln \left(\frac{z \bar{z}}{\mathfrak{a}^{2}}\right)+\cdots
\end{gathered}
$$

$$
\begin{aligned}
\left\langle\chi_{a}^{*}(z, \bar{z}) \chi_{b}(0,0)\right\rangle_{\mathcal{L}_{1}, g_{M}, 0} & =-\left\langle\chi_{a}(z, \bar{z}) \chi_{b}^{*}(0,0)\right\rangle_{\mathcal{L}_{1}, g_{M}, 0} \\
& =-\delta_{a b} \zeta \ln \left(\frac{z \bar{z}}{\mathfrak{a}^{2}}\right)
\end{aligned}
$$

$(a=1,2)$, respectively. There are nonvanishing correlation functions between $\phi$ 's and their dual $\widetilde{\phi}$ 's but we will not need them. Here, the ellipses means that short-distance contributions such as $\delta$ functions have been omitted. Observe that Eqs. (5.15a) and (5.15b) apply not only to $\mathcal{L}_{1}$ with $g_{A}$ $=0$ but also to $\mathcal{L}$ with $g_{A} \geqslant 0$. However, we stress that Eqs. (5.15a) and (5.15b) were first computed with $g_{A}=0$. The full $g_{A}$ dependence was then uncovered through the chiral transformation (5.9). Another important point to keep in mind is that the squared logarithmic term enters with a negative sign in the two-point functions for the fields $\phi_{a}$ and $\phi_{a}^{\prime}$, while it enters with a positive sign for the dual fields $\widetilde{\phi}_{a}$ and $\widetilde{\phi}_{a}^{\prime}$.

(ii) Many-point correlation functions among $\phi_{a}, \phi_{a}^{\prime}, \widetilde{\phi}_{a}$, $\widetilde{\phi}_{a}^{\prime}, \chi_{a}^{*}, \chi_{a}$ can be reduced to the two-point correlation functions (5.15) with the help of Wick's theorem. Applicability of Wick's theorem is specific to $\mathcal{L}_{1}$ with $g_{A}=0$. 
The $\beta$ functions in Eqs. (5.2) for $g_{A}$ and $g_{M}$ follow from Eqs. (5.15a) or (5.15b) by demanding that these two-point functions be invariant under rescaling (4.4) of the lattice spacing $\mathfrak{a}$. It is the presence of the squared logarithm terms in Eqs. (5.15) that spoils the conformal symmetry of the MDH model, i.e., the theory with $g_{A} \geqslant 0$ and $g_{M}=0$. The damages caused to conformal invariance are minimal, however, since it remains possible to use Wick's theorem perturbatively and since the dependence on $g_{A}$ in correlation functions is exact to lowest order in perturbation theory.

\section{B. Operator content at near criticality}

We have seen in Sec. IV A that the energy perturbation (4.2) induces local composite operators given by Eq. (4.7a) with the anomalous scaling dimensions (4.7b) in the MDH model. We are going to show that the dependence on $g_{M}$ of the anomalous scaling dimensions of these operators can be calculated perturbatively in the HWK model whereas the dependence on $g_{A}$ is, up to the positive multiplicative factor $\zeta^{2}$, exact in Eq. (4.7b). Hence, it is imperative to account for the most relevant of these operators in a consistent way when writing the Callan-Symanzik equation for the DOS.

To begin with, we must bosonize the eight Cooper terms entering in the energy perturbation (4.2). This is done with the help of Table V from which we infer that only the four linear combinations

$$
\Theta_{ \pm}:=\frac{1}{2}\left[\left(\widetilde{\phi}_{1}+\widetilde{\phi}_{2}\right) \pm\left(\phi_{1}-\phi_{2}\right)\right]
$$

and

$$
\Theta_{ \pm}^{\prime}:=\frac{1}{2}\left[\left(\widetilde{\phi}_{1}^{\prime}+\widetilde{\phi}_{2}^{\prime}\right) \pm\left(\phi_{1}^{\prime}-\phi_{2}^{\prime}\right)\right]
$$

enter in the vertex operators representing the Cooper terms. Observe that the energy perturbation depends on the dual fields after Abelian bosonization.

Permutation symmetry of the flavor indices at the band center simplifies considerably the evaluation of two-point functions between $\Theta$ 's. In particular, two-point functions between scalar fields and their dual cancel each other. Alternatively, this is so because the conformal spin of the energy perturbation vanishes; i.e., there are as many left as right movers in any of the monomials making up the energy perturbing operator.

With the help of Eqs. (5.15a) and (5.15b) we can evaluate exactly all two-point functions listed in the first column of Table VI. For the purpose of computing scaling dimensions, these exact two-point functions are only the zeroth-order contribution to the expectation value of products of vertex operators in perturbation theory in powers of $\mathcal{L}_{2}$. They are given in the second column of Table VI. Observe that the
TABLE V. Abelian bosonization rules for the eight Cooper terms entering the energy perturbation (4.2). The fields $\phi_{a}, \varphi_{a-}$, and $\varphi_{a+}$ are related by $\phi_{a}(z, \bar{z})=\varphi_{a-}(z)+\varphi_{a+}(\bar{z})$. The fields $\phi_{a}^{\prime}$, $\varphi_{a-}^{\prime}$, and $\varphi_{a+}^{\prime}$ are related by $\phi_{a}^{\prime}(z, \bar{z})=\varphi_{a-}^{\prime}(z)+\varphi_{a+}^{\prime}(\bar{z})$. The fields $\widetilde{\phi}_{a}, \varphi_{a-}$, and $\varphi_{a+}$ are related by $\widetilde{\phi}_{a}(z, \bar{z})=\varphi_{a-}(z)$ $-\varphi_{a+}(\bar{z})$. The fields $\widetilde{\phi}_{a}^{\prime}, \varphi_{a-}^{\prime}$, and $\varphi_{a+}^{\prime}$ are related by $\widetilde{\phi}_{a}^{\prime}(z, \bar{z})$ $=\varphi_{a-}^{\prime}(z)-\varphi_{a+}^{\prime}(\bar{z})$. The lattice spacing $\mathfrak{a}$ is used as a shortdistance cutoff.

\begin{tabular}{lr}
\hline \hline SUSY & Bosonized Cooper terms \\
\hline$\psi_{1-}^{*} \psi_{2+}^{*}$ & $\mathfrak{a}^{-1} \exp \left\{-\frac{i}{2}\left[\left(\widetilde{\phi}_{1}+\widetilde{\phi}_{2}\right)+\left(\phi_{1}-\phi_{2}\right)\right]\right\}$ \\
$\psi_{1+}^{*} \psi_{2-}^{*}$ & $\mathfrak{a}^{-1} \exp \left\{-\frac{i}{2}\left[\left(\widetilde{\phi}_{1}+\widetilde{\phi}_{2}\right)-\left(\phi_{1}-\phi_{2}\right)\right]\right\}$ \\
$\beta_{1-}^{*} \beta_{2+}^{*}$ & $\mathfrak{a} \exp \left\{-\frac{i}{2}\left[\left(\widetilde{\phi}_{1}^{\prime}+\widetilde{\phi}_{2}^{\prime}\right)+\left(\phi_{1}^{\prime}-\phi_{2}^{\prime}\right)\right]\right\}\left(i \partial \chi_{1}\right)\left(i \bar{\partial} \chi_{2}\right)$ \\
$\beta_{1+}^{*} \beta_{2-}^{*}$ & $\mathfrak{a} \exp \left\{-\frac{i}{2}\left[\left(\widetilde{\phi}_{1}^{\prime}+\widetilde{\phi}_{2}^{\prime}\right)-\left(\phi_{1}^{\prime}-\phi_{2}^{\prime}\right)\right]\right\}\left(i \bar{\partial} \chi_{1}\right)\left(i \partial \chi_{2}\right)$ \\
$\psi_{2+} \psi_{1-}$ & $\mathfrak{a}-1 \exp \left\{+\frac{i}{2}\left[\left(\widetilde{\phi}_{1}+\widetilde{\phi}_{2}\right)+\left(\phi_{1}-\phi_{2}\right)\right]\right\}$ \\
$\psi_{2-} \psi_{1+}$ & $\mathfrak{a}-1 \exp \left\{+\frac{i}{2}\left[\left(\widetilde{\phi}_{1}+\widetilde{\phi}_{2}\right)-\left(\phi_{1}-\phi_{2}\right)\right]\right\}$ \\
$\beta_{2+} \beta_{1-}$ & $\mathfrak{a} \exp \left\{+\frac{i}{2}\left[\left(\widetilde{\phi}_{1}^{\prime}+\widetilde{\phi}_{2}^{\prime}\right)+\left(\phi_{1}^{\prime}-\phi_{2}^{\prime}\right)\right]\right\}\left(i \bar{\partial} \chi_{2}^{*}\right)\left(i \partial \chi_{1}^{*}\right)$ \\
$\beta_{2-} \beta_{1+}$ & $\mathfrak{a} \exp \left\{+\frac{i}{2}\left[\left(\widetilde{\phi}_{1}^{\prime}+\widetilde{\phi}_{2}^{\prime}\right)-\left(\phi_{1}^{\prime}-\phi_{2}^{\prime}\right)\right]\right\}\left(i \partial \chi_{2}^{*}\right)\left(i \bar{\partial} \chi_{1}^{*}\right)$ \\
\hline \hline
\end{tabular}

dependence on the squared logarithm and hence on $g_{A}$ (through the chiral transformation) arises solely from the dual sector of the theory as the contributions from the nondual sector always cancel. This is consistent with the fact that the random vector potential only couples to the dual field. In the present formalism, this follows from the fact that the center of mass drops out in the difference of any of the coordinates $\phi_{a}$ or $\phi_{a}^{\prime}$. The dependence on $g_{A}$ extracted from Table VI is exact for the scaling dimensions of vertex operators in that perturbation theory only changes the linear dependence on the logarithm through corrections that depend solely on $g_{M}$. Observe that vertex operators corresponding to products of $\beta$ 's follow from two-point functions with prime fields. Hence, the contribution to the term linear in the logarithm that does not depend on $g_{A}$ has the "wrong sign." In the absence of a random mass, this wrong sign is corrected by accounting for the contribution to the correlation functions from the $\chi$ sector [see Eq. (4.7b) and Appendix B]. In the presence of a random mass, the correction to this "wrong sign" from the $\chi$ sector is only partial [see Eq. (5.18)].

The central result of this section is the evaluation of the anomalous scaling dimensions $x_{\mathbf{m}, \mathbf{n}}$ for the local composite operators defined by Eq. (4.7a). To zeroth order in the perturbation theory defined in Sec. V A, they are given by 
TABLE VI. Evaluation of all two-point functions governing scaling dimensions appearing in OPE's of energy perturbation with itself. The ellipses refer to $\delta$ function contributions to the two-point functions. Although these two-point functions are exact, they yield an approximation to the calculation of scaling dimensions. Nevertheless, the dependence on $g_{A}$ of scaling dimensions is exact. The dependence of linear logarithmic term on $g_{M}$ that does not depend on $g_{A}$ is modified by residual interactions in $\mathcal{L}_{2}$ when computing scaling dimensions.

$$
\begin{aligned}
& \left\langle\Theta_{+}(z, \bar{z}) \Theta_{+}(0,0)\right\rangle_{\mathcal{L}, g_{M}, g_{A}} \\
& \zeta\left(-1+\frac{g_{A} \zeta}{\pi}\right) \ln \left(\frac{z \bar{z}}{\mathfrak{a}^{2}}\right)+\left(\frac{g_{M} \zeta^{2}}{2 \pi}\right)^{2} \ln ^{2}\left(\frac{z \bar{z}}{\mathfrak{a}^{2}}\right)+\cdots \\
& \left\langle\Theta_{-}(z, \bar{z}) \Theta_{-}(0,0)\right\rangle_{\mathcal{L}, g_{M}, g_{A}} \\
& \left\langle\Theta_{+}(z, \bar{z}) \Theta_{-}(0,0)\right\rangle_{\mathcal{L}, g_{M}, g_{A}} \\
& \left\langle\Theta_{-}(z, \bar{z}) \Theta_{+}(0,0)\right\rangle_{\mathcal{L}, g_{M}, g_{A}} \\
& \left\langle\Theta_{+}^{\prime}(z, \bar{z}) \Theta_{+}^{\prime}(0,0)\right\rangle_{\mathcal{L}, g_{M}, g_{A}} \\
& \left\langle\Theta_{-}^{\prime}(z, \bar{z}) \Theta_{-}^{\prime}(0,0)\right\rangle_{\mathcal{L}, g_{M}, g_{A}} \\
& \left\langle\Theta_{+}^{\prime}(z, \bar{z}) \Theta_{-}^{\prime}(0,0)\right\rangle_{\mathcal{L}, g_{M}, g_{A}} \\
& \left\langle\Theta_{-}^{\prime}(z, \bar{z}) \Theta_{+}^{\prime}(0,0)\right\rangle_{\mathcal{L}, g_{M}, g_{A}} \\
& \left\langle\Theta_{+}^{\prime}(z, \bar{z}) \Theta_{+}(0,0)\right\rangle_{\mathcal{L}, g_{M}, g_{A}} \\
& \left\langle\Theta_{-}^{\prime}(z, \bar{z}) \Theta_{-}(0,0)\right\rangle_{\mathcal{L}, g_{M}, g_{A}} \\
& \left\langle\Theta_{+}^{\prime}(z, \bar{z}) \Theta_{-}(0,0)\right\rangle_{\mathcal{L}, g_{M}, g_{A}} \\
& \left\langle\Theta_{-}^{\prime}(z, \bar{z}) \Theta_{+}(0,0)\right\rangle_{\mathcal{L}, g_{M}, g_{A}} \\
& \text { as above } \\
& \frac{g_{A} \zeta^{2}}{\pi} \ln \left(\frac{z \bar{z}}{\mathfrak{a}^{2}}\right)+\left(\frac{g_{M} \zeta^{2}}{2 \pi}\right)^{2} \ln ^{2}\left(\frac{z \bar{z}}{\mathfrak{a}^{2}}\right)+\cdots \\
& \text { as above } \\
& \zeta\left(+1+\frac{g_{A} \zeta}{\pi}\right) \ln \left(\frac{z \bar{z}}{\mathfrak{a}^{2}}\right)+\left(\frac{g_{M} \zeta^{2}}{2 \pi}\right)^{2} \ln ^{2}\left(\frac{z \bar{z}}{\mathfrak{a}^{2}}\right)+\cdots \\
& \text { as above } \\
& \frac{g_{A} \zeta^{2}}{\pi} \ln \left(\frac{z \bar{z}}{\mathfrak{a}^{2}}\right)+\left(\frac{g_{M} \zeta^{2}}{2 \pi}\right)^{2} \ln ^{2}\left(\frac{z \bar{z}}{\mathfrak{a}^{2}}\right)+\ldots \\
& \text { as above } \\
& \frac{g_{A} \zeta^{2}}{\pi} \ln \left(\frac{z \bar{z}}{\mathfrak{a}^{2}}\right)+\left(\frac{g_{M} \zeta^{2}}{2 \pi}\right)^{2} \ln ^{2}\left(\frac{z \bar{z}}{\mathfrak{a}^{2}}\right)+\cdots \\
& \text { as above } \\
& \text { as above } \\
& \text { as above } \\
& x_{\mathbf{m}, \mathbf{n}}=\left[\left(m_{12+}-m_{12-}\right)^{2}+\left(m_{21+}-m_{21-}\right)^{2}-\left(n_{12+}-n_{12-}\right)^{2}-\left(n_{21+}-n_{21-}\right)^{2}\right] \zeta\left[1+\mathcal{O}\left(g_{M}\right)\right] \\
& -\left[\left(m_{12+}-m_{12-}\right)+\left(m_{21+}-m_{21-}\right)+\left(n_{12+}-n_{12-}\right)+\left(n_{21+}-n_{21-}\right)\right]^{2} \frac{g_{A}}{\pi} \zeta^{2} \\
& +\left(n_{12+}-n_{12-}\right)^{2}+\left(n_{21+}-n_{21-}\right)^{2}+\left|n_{12+}-n_{12-}\right|+\left|n_{21+}-n_{21-}\right|+\mathcal{O}\left(g_{M}\right)
\end{aligned}
$$

as the expectation value of any product of vertex operators can be replaced by the exponential of the properly weighted sum of all 12 nonvanishing two-point functions listed in Table VI. Anomalous scaling dimensions are then extracted from the coefficient to the term linear in $\ln \left(\bar{z} z / \mathfrak{a}^{2}\right)$. One should not forget the contribution to the anomalous scaling dimension coming from the $\chi$ 's which is responsible for the third line in Eq. (5.18). What makes Eq. (5.18) remarkable is that even if $\mathcal{O}_{\mathbf{m}, \mathbf{n}}$ is not relevant at $g_{M}=0$, switching on a mass perturbation renders $\mathcal{O}_{\mathbf{m}, \mathbf{n}}$ relevant at sufficiently large length scales since $g_{A}$ is marginally relevant (in the sense that it grows logarithmically with length) whereas $g_{M}$ is exactly marginal. Moreover, higher-order corrections from perturbation theory do not invalidate this argument. The strategy to deal with this difficulty was described in Sec. IV. We are going to generalize the RG analysis of Sec. IV to account for the relevance of $g_{A}$. As before we select the operators (4.9) since they are the most relevant operators for given $N$ in Eq. (4.8) although it must be noted that their scaling dimensions are not anymore degenerate for finite $g_{M}$. The lifting of the degeneracy (4.10) is negligible, however, since it is of order $g_{M} / g_{A}$ whereby $g_{M}$ does not flow but $g_{A}$ flows to infinity in the infrared limit.

\section{Callan-Symanzik equation for DOS}

The Callan-Symanzik equation obeyed by the DOS in the HWK model is given by

$$
0=\left(\sum_{N=1}^{\infty} \beta_{Y_{N}} \frac{\partial}{\partial Y_{N}}+\beta_{g_{M}} \frac{\partial}{\partial g_{M}}+\beta_{g_{A}} \frac{\partial}{\partial g_{A}}-x_{1}\right) \nu(\varepsilon),
$$

where the $\beta$ functions for the fugacities are

$$
\beta_{Y_{N}}=\left(2-x_{N}\right) Y_{N}+\pi \sum_{N^{\prime}=1}^{N-1}\left(\begin{array}{c}
N \\
N^{\prime}
\end{array}\right) Y_{N^{\prime}} Y_{N-N^{\prime}}
$$




$$
\begin{gathered}
x_{N}=\zeta\left[1+\mathcal{O}\left(g_{M}\right)\right] N-\left[\frac{g_{A}}{\pi} \zeta^{2}+\mathcal{O}\left(g_{M}\right)\right] N^{2}, \\
Y_{N}(l=0)=\frac{\varepsilon-i \eta}{\mathfrak{a}^{x_{1}-2}} \delta_{1, N},
\end{gathered}
$$

the $\beta$ function for the variance of the random mass vanishes, and the $\beta$ function for the variance of the random vector potential is

$$
\beta_{g_{A}}=\frac{\left(g_{M} \zeta\right)^{2}}{2 \pi^{2}}
$$

[Remember that $\beta_{g_{M}}$ and $\beta_{g_{A}}$ follow from demanding that the two-point functions Eqs. (5.15a) or (5.15b) be invariant under rescaling (4.4) of the lattice spacing a.] The function $\zeta$ is given in Eq. (5.2). We made the same approximations to reach Eq. (5.19) as we did to obtain Eq. (4.17); i.e., we ignored renormalization effects induced on $g_{M}$ and $g_{A}$ by the fugacities and we ignored annihilation processes when deriving the RG equations obeyed by the fugacities. Furthermore, we work to lowest order in $g_{M}$ as $g_{M}$ does not flow whereas $g_{A}$ flows to infinity in the infrared limit. The infinite set of RG equations (5.19b) obeyed by the fugacities can be recast as a second-order nonlinear partial differential equation of the KPP type

$$
\begin{gathered}
\partial_{l} \widetilde{G}=\left(\zeta \partial_{y}+\frac{g_{A} \zeta^{2}}{\pi} \partial_{y}^{2}\right) \widetilde{G}+2 \widetilde{G}(\widetilde{G}-1), \\
\widetilde{G}(y, l)=1+\frac{\pi}{2} \sum_{n=1}^{\infty} \frac{(-1)^{n} e^{-n y}}{n !} Y_{n}(l), \\
\widetilde{G}(y, 0)=\exp \left(-\frac{\pi}{2} \frac{\varepsilon-i \eta}{\mathfrak{a}^{x_{1}-2}} e^{-y}\right) .
\end{gathered}
$$

The notable difference with Eq. (4.22) is the scale dependence of the diffusion term $g_{A}(l) \zeta^{2} / \pi$.

We tentatively define the dynamical exponent $z_{A}$ for the HWK model by

$$
z_{A}:= \begin{cases}2-\zeta+\frac{g_{A}}{\pi} \zeta^{2}+\mathcal{O}\left(g_{M}\right), & \text { for } g_{A} \zeta^{2}<2 \pi, \\ 4 \sqrt{\frac{g_{A}}{2 \pi}} \zeta-\zeta-\mathcal{O}\left(g_{M}\right), & \text { for } g_{A} \zeta^{2} \geqslant 2 \pi .\end{cases}
$$

The dynamical exponent $z_{A}$ in Eq. (5.21a) would be the velocity of the wave front of the Callan-Symanzik equation (5.20) if the diffusion constant was scale independent. However, the dependence of the diffusion constant $\propto g_{A}(l)$ on the rescaling parameter $l$ is linear whereas that of $\widetilde{G}(y, l)$ is exponential. We can thus make an adiabatic approximation by which the Callan-Symanzik equation for the DOS (4.37) becomes

$$
0=\left[z_{A} \varepsilon \frac{\partial}{\partial \varepsilon}+\beta_{g_{A}} \frac{\partial}{\partial g_{A}}-\left(2-z_{A}\right)\right] \nu(\varepsilon) .
$$

The Callan-Symanzik equation (5.21) is the central result of our paper. It differs from the Callan-Symanzik equation of Guruswamy et al. in Ref. 27 through the freezing transition of the dynamical exponent in Eq. (5.21a).

\section{DOS}

The formal solution to Eq. (5.21) is

$$
\begin{gathered}
\nu(l)=\nu(0) \exp \left[+\int_{0}^{l} d l\left(2-z_{A}\right)\right], \\
d l=\frac{2 \pi^{2}}{\left(g_{M} \zeta\right)^{2}} d g_{A}, \\
z_{A} d l=\frac{d \varepsilon}{\varepsilon} .
\end{gathered}
$$

With the notation $\varepsilon_{\text {ren }} \equiv \varepsilon(l), \varepsilon \equiv \varepsilon(0)$, we find

$$
\frac{\nu(\varepsilon)}{\nu\left(\varepsilon_{\mathrm{ren}}\right)}=\left(\frac{\varepsilon_{\mathrm{ren}}}{\varepsilon}\right) e^{-\left[4 \pi^{2} /\left(g_{M} \zeta\right)^{2}\right]\left[g_{A}\left(\varepsilon_{\mathrm{ren}}\right)-g_{A}(\varepsilon)\right]},
$$

where $g_{A}\left(\varepsilon_{\text {ren }}\right)-g_{A}(\varepsilon)$ is the solution to

$$
\ln \left(\frac{\varepsilon_{\mathrm{ren}}}{\varepsilon}\right)=\frac{2 \pi^{2}}{\left(g_{M} \zeta\right)^{2}} \int_{g_{A}(\varepsilon)}^{g_{A}\left(\varepsilon_{\mathrm{ren}}\right)} d g_{A} z_{A} .
$$

The renormalized energy scale $\varepsilon_{\text {ren }} \equiv \varepsilon(l)$ is the energy scale at which we cannot neglect renormalization effects from the energy perturbation on the variances of the random potentials anymore. Beyond the energy scale $\varepsilon_{\text {ren }}$, the RG analysis encoded by the Callan-Symanzik equation (5.21) breaks down. We take $\varepsilon_{\text {ren }}$ as given, but we allow $l$ to be tuned, or, equivalently, we take the bare energy $\varepsilon \equiv \varepsilon(0)$ to be tunable. How large the rescaling scale $l$ is thus dictates how close the bare energy scale $\varepsilon$ is to the band center. The infrared limit $l$ $\rightarrow \infty$ gives the DOS arbitrarily close to the band center.

Equation (5.23a) implies that the DOS is characterized by two regimes each of which can itself be divided into two subregimes. When

$$
\frac{g_{A}(l)-g_{A}(0)}{g_{A}(0)} \ll 1,
$$

the scale dependence of $g_{A}$ can be neglected in the CallanSymanzik equation (5.21b). We then recover the DOS for the MDH model (4.38) with $z_{A}$ given by Eq. (5.21a), whereby the freezing transition can be observed as a function of the bare energy $\varepsilon$ depending on whether $g_{A}(\varepsilon) \zeta^{2}>2 \pi$ or $g_{A}(\varepsilon) \zeta^{2}<2 \pi$. When

$$
\frac{g_{A}(l)-g_{A}(0)}{g_{A}(0)} \gtrsim 1,
$$


the scale dependence of $g_{A}$ in the Callan-Symanzik equation (5.21b) is dominant. In the infrared limit $l \rightarrow \infty$, i.e., arbitrarily close to the band center, the integral on the right-hand side of Eq. (5.23b) is always dominated by the contribution

$$
\int_{2 \pi / \zeta^{2}}^{g_{A}\left(\varepsilon_{\mathrm{ren}}\right)} d g_{A} z_{A} \approx\left[\frac{8}{3} \sqrt{\frac{g_{A}\left(\varepsilon_{\mathrm{ren}}\right)}{2 \pi}} \zeta-\zeta-\mathcal{O}\left(g_{M}\right)\right] g_{A}\left(\varepsilon_{\mathrm{ren}}\right) .
$$

After solving Eq. (5.23b) for the leading dependence of $g_{A}$ on $\varepsilon_{\text {ren }}$, this gives the DOS

$$
\frac{\nu(\varepsilon)}{\nu\left(\varepsilon_{\text {ren }}\right)} \sim\left(\frac{\varepsilon_{\text {ren }}}{\varepsilon}\right) \exp \left[-c\left|\ln \left(\frac{\varepsilon_{\text {ren }}}{\varepsilon}\right)\right|^{2 / 3}\right],
$$

where $c$ is some nonuniversal positive constant. Observation of the Gade singularity

$$
\frac{\nu(\varepsilon)}{\nu\left(\varepsilon_{\text {ren }}\right)} \sim\left(\frac{\varepsilon_{\text {ren }}}{\varepsilon}\right) \exp \left[-c\left|\ln \left(\frac{\varepsilon_{\text {ren }}}{\varepsilon}\right)\right|^{1 / 2}\right],
$$

demands fine-tuning of bare and renormalized $g_{A}$ 's so as to avoid the freezing transition in Eq. (5.21a). At best, the Gade singular DOS can be observed as a crossover in a finite window of energy.

\section{CONCLUSIONS}

Two recent theoretical works have addressed the issue of the distribution of the DOS in the HWK model. Guruswamy et al. in Ref. 27 considered a narrow distribution of disorder at the microscopic level for which the use of field-theoretical methods at longer length scales is justified. They computed the DOS within a SUSY field theory and found that it displays the Gade singularity $\varepsilon^{-1} \exp \left(-c|\ln \varepsilon|^{1 / 2}\right)$ in the neighborhood of the band center. Motrunich et al. in Ref. 30, bypassing a purely field-theoretical approach, came to the conclusion that the DOS is given by $\varepsilon^{-1} \exp \left(-c|\ln \varepsilon|^{2 / 3}\right)$ instead of the Gade singularity. Our field-theoretical analysis agrees with the prediction of Motrunich et al.

We find that the RG analysis performed on the DOS by Guruswamy et al. is not consistent as it overlooked the existence of infinitely many operators with negative anomalous scaling dimensions. The physical interpretation of these operators is that the LDOS is a very broadly distributed random variable close to the band center. The situation here shares many similarities with the scaling with system size of the distribution of the conductance in 2D mesoscopic samples which is known to broaden with increasing system size (i.e., by moving away from the diffusive regime) ${ }^{57}$ A parallel with quasi-one-dimensional wires is particularly instructive. In quasi-one-dimensions, it is known that the infinite set of scaling equations for the moments of the conductance can be traded for a Fokker-Planck equation obeyed by the underlying distribution of the conductance. ${ }^{58}$ The advantage of the Fokker-Planck equation over the scaling equations for the moments is that the Fokker-Planck equation can be solved in the localized regime (i.e., when the length of the sample diverges). It is then possible to show that the typical conductance is self-averaging (at least in the standard universality classes of Anderson localization) in the localized regime. ${ }^{59}$ Similarly, all moments of the LDOS are here strongly coupled through infinitely many RG equations encoding the dependence on energy arbitrarily close to the band center. The Gade singularity results from an inconsistent truncation of this infinite set of coupled equations to a finite subset. As with the quasi-one-dimensional conductance, it is possible to trade the infinite set of scaling equations for the DOS in favor of a second-order partial differential equation obeyed by the underlying probability distribution for the normalization of zero modes from which one can extract the dependence on energy of the DOS. As with the quasi-onedimensional typical conductance in the standard universality classes of Anderson localization, the DOS is well behaved statistically; i.e., it is self-averaging in the infrared limit.

A classification of random Hamiltonians with short-rangecorrelated disorder in terms of intrinsic symmetries in conjunction with scaling concepts has emerged, since its inception in the late 1970's, as perhaps one of the deepest insight into the physics of Anderson localization. The HWK model is invariant under time reversal and spin rotation. Those are the standard symmetries in Anderson localization. The HWK model also preserves a bipartite lattice symmetry, making it a member of the chiral orthogonal universality class. The HWK model is nevertheless special since the disorder is parametrized (in a way consistent with rescaling of the lattice spacing) by only two independent variances as opposed to four for a generic member of the chiral orthogonal universality class. Since the works of Gade and Wegner, it is believed that the DOS in the chiral orthogonal universality class displays the Gade singularity. If universality is indeed the only ingredient that determines the scaling exponent $v$ and $\kappa$ for the singular density

$$
|\varepsilon|^{-v} \exp \left(-c|\ln \varepsilon|^{\kappa}\right)
$$

in the chiral orthogonal universality class, we must conclude that the RG analysis made by Gade in Ref. 8 suffers from the same deficiencies as the RG analysis of Guruswamy et al.

This possibility is perhaps not so surprising if we take the existence of infinitely many operators with negative anomalous scaling dimensions as the "smoking gun" for broadly distributed random variables. There have been indications since the early 1980's that replicated NLSM might support infinitely many operators with negative anomalous scaling dimensions. ${ }^{60}$ A perhaps stronger clue comes from the interplay between multifractality of zero modes and the broad distribution of the LDOS in the HWK model. As we have shown explicitly in this paper, essentially the same spectrum of negative scaling dimensions controls the multifractal spectrum of zero modes in the MDH model and the CallanSymanzik equation for the DOS near the band center in the HWK model. Fal'ko and Efetov have searched for and found in the diffusive regime of 2D disordered metals the signature of multifractal states. ${ }^{61}$ There are states, dubbed prelocalized states, that are characterized by very large amplitude fluctuations whose statistics are well described (in a finite window of length scales) by a log-normal distribution, i.e., by the multifractal spectrum of zero modes for a Dirac spinor subjected to a random vector potential. ${ }^{39,61}$ It could very well be 
that the $\operatorname{gl}(1 \mid 1)$ current algebra at the heart of the freezing transition in the multifractal spectrum of zero modes in the MDH model and for the DOS of the HWK model, although broken by all the additional degrees of freedom encoded by NLSM in the standard or chiral universality classes, is sufficiently robust to reveal itself in those latter models in connection with prelocalized states and broadly distributed LDOS, respectively. To put it differently, the critical statistics of wave functions might still be log normal even though the wave functions have a much more complicated structure than a single exponential of a field with logarithmic spatial correlations. To add credit to this speculation, we know of one example where critical modes have a much more complex structure than that of a single exponential of a twodimensional scalar field (a Gaussian random surface in the terminology of Motrunich et al.) although their multifractal spectrum is still of the Gaussian random surface type: $N$ flavors of Dirac fermions subjected to a random $\mathrm{SU}(N)$ gauge potential. ${ }^{37,62}$ An interesting direction that might be worth exploring is the construction of variational methods that would generalize to the NLSM the one devised by Horovitz and Le Doussal in Ref. 42 to capture the nonperturbative physics of freezing in the 2D random phase $X Y$ model.

Another intriguing question is whether multifractality of critical states at a mobility edge or at the critical energy in the quantum Hall plateau transition necessarily reveals itself in a broad distribution of the LDOS. If the answer is positive as is suggested by numerical simulations in Refs. 63 and 64, the LDOS might be more interesting than commonly thought. Indeed, it has been proposed that the freezing transition in the multifractal spectrum of zero modes in the HWK model is generic to multifractal wave functions at a mobility edge and, in particular, also applies to critical states in the plateau transition. ${ }^{65}$ The corollary would then be that a counterpart to this freezing transition characterizes the statistical distribution of the LDOS - say, that typical and average LDOS disagree. So far, relatively little attention has been paid to the distribution of the LDOS in the plateau transition since the calculation by Wegner of the DOS (Ref. 66) in spite of great theoretical efforts to identify the critical theory governing the plateau transition (see Refs. 67 and 68 for the latest endeavors).

Note added in proof. Within the SUSY representation of the Gade model (a NLSM) introduced by Guruswamy et al., it can be seen that it is the so-called Gade term which is, ultimately, responsible for the freezing transition of the DOS. The Gade term in the Lagrangian of the NLSM results from the fact that the superdeterminant of the supermatrix $T$ entering the NLSM is not constrained to some fixed numerical value as is the case in all other universality classes. The superdeterminant of $T$ is thus a dynamical variable of its own whose fluctuations are controlled by the Gade term $\left(\partial_{\mu} \ln \operatorname{Sdet} T\right)^{2}$. A similar role is played by the Gade term in the bosonic and fermionic replicated NLSM representing the chiral universality classes.

\section{ACKNOWLEDGMENTS}

We would like to thank A. W. W. Ludwig for pointing out to us how the ambiguity in the bosonization rules of the gl(1|1) super current algebra is lifted by imposing boundary conditions on the bosonized fields. This work was supported in part by a Grant-in-Aid for Scientific Research on Priority Areas (A) from the Ministry of Education, Culture, Sports, Science and Technology (Grant No. 12046238) (A.F.) and JSPS (S.R.). C.M. thanks the Yukawa Institute for Theoretical Physics in Kyoto for its hospitality during the initial stage of this work.

\section{APPENDIX A: THE PROCESS OF COMPLETE ANNIHILATION}

In this appendix, we give the full OPE's obeyed by the composite operators that control the DOS in the MDH model of Sec. IV. Although this paper deals with two flavors of Dirac fermions subjected to an imaginary random vector potential and a complex-valued random mass, we also consider the case of one flavor subjected to a real-valued random vector potential. ${ }^{48}$ Indeed, as we have argued, the problem of the DOS in the two-flavor problem reduces to a large extent to the one-flavor problem. Moreover, the one-flavor problem is interesting in its own right as it is related to the plateau transition in the integer quantum Hall effect ${ }^{9}$ and to the $2 \mathrm{D}$ random phase $X Y$ model. $27,35,37,41$

\section{Case of one flavor}

The problem of a single flavor of Dirac fermions coupled to a real-valued random vector potential can be shown to reduce to the action ${ }^{37}$

$$
\begin{aligned}
S_{*}= & \frac{1}{2 g_{A}} \int d^{2} \boldsymbol{r}\left(\partial_{\mu} \Phi_{1}\right)^{2}+\int \frac{d^{2} \boldsymbol{r}}{\pi}\left(\psi_{-}^{*} \bar{\partial} \psi_{-}+\psi_{+}^{*} \partial \psi_{+}\right) \\
& +\int \frac{d^{2} \boldsymbol{r}}{\pi}\left(\beta_{-}^{*} \bar{\partial} \beta_{-}+\beta_{+}^{*} \partial \beta_{+}\right) .
\end{aligned}
$$

Here, $\Phi_{1}$ is the transversal component of the random vector potential, with variance $g_{A}$. For simplicity, the longitudinal part $\Phi_{2}$ and the Jacobian that arise when trading the vector potential by its transversal and longitudinal components (conformal sector with net central charge -1 ) have been dropped from the action as we shall only seek the scaling dimensions of gauge invariant composite operators. Action (A1) is critical for arbitrary value of $g_{A}$ as shown in Ref. 9 and defines a CFT with gl(1|1) symmetry as shown in Ref. 37.

It was shown in Ref. 37 that there exists an infinite set of operators with negative anomalous scaling dimensions. These operators are composite operator of the form

$$
\begin{aligned}
\mathcal{O}_{\mathbf{m}, \mathbf{n}}= & \left(\mathcal{F}_{+}\right)^{m_{+}}\left(\mathcal{B}_{+}\right)^{n_{+}}\left(\mathcal{F}_{-}\right)^{m_{-}}\left(\mathcal{B}_{-}\right)^{n_{-}} \\
& \times \exp \left[2\left(m_{+}+n_{+}-m_{-}-n_{-}\right) \Phi_{1}\right],
\end{aligned}
$$

where $\mathcal{F}_{ \pm}$and $\mathcal{B}_{ \pm}$are defined as

$$
\begin{array}{ll}
\mathcal{F}_{+}:=\psi_{-}^{*} \psi_{+}, & \mathcal{F}_{-}:=\psi_{+}^{*} \psi_{-}, \\
\mathcal{B}_{+}:=\beta_{-}^{*} \beta_{+}, & \mathcal{B}_{-}:=\beta_{+}^{*} \beta_{-} .
\end{array}
$$

Their scaling dimensions are given by 


$$
\begin{aligned}
x_{\mathbf{m}, \mathbf{n}}= & \left(m_{+}-m_{-}\right)^{2}+\left|n_{+}-n_{-}\right| \\
& -\frac{g_{A}}{\pi}\left[\left(m_{+}-m_{-}\right)+\left(n_{+}-n_{-}\right)\right]^{2},
\end{aligned}
$$

respectively. We thus see that for any value of the disorder strength $g_{A}$, there are infinitely many operators with negative scaling dimensions as $\left(n_{+}-n_{-}\right)^{2}$ dominates over $\mid n_{+}$ $-n_{-} \mid$for large $\left|n_{+}-n_{-}\right|$. Of all the composite operators $\left\{\mathcal{O}_{\mathbf{m}, \mathbf{n}}\right\}$, we focus on the subset spanned by

$$
\left(\mathcal{B}_{ \pm}\right)^{N} e^{ \pm 2 N \Phi_{2}}, \quad\left(\mathcal{F}_{ \pm}\right)\left(\mathcal{B}_{ \pm}\right)^{N-1} e^{ \pm 2 N \Phi_{2}}
$$

where $N$ is a positive integer. For any given $N$, these two operators are the most relevant and share the same scaling dimension, forming a two-dimensional representation of gl(1|1). We then construct gl(1|1) singlets $^{37}$

$$
\begin{aligned}
& \mathcal{A}_{+; N}:=\frac{e^{+2 N \Phi_{1}}}{N !}\left(\mathcal{B}_{+}^{N}+N \mathcal{F}_{+} \mathcal{B}_{+}^{N-1}\right), \\
& \mathcal{A}_{-; N}:=\frac{e^{-2 N \Phi_{1}}}{N !}\left(\mathcal{B}_{-}^{N}+N \mathcal{F}_{-} \mathcal{B}_{-}^{N-1}\right),
\end{aligned}
$$

whose scaling dimensions read

$$
x_{ \pm ; N}=x_{N}=N-\frac{g_{A}}{\pi} N^{2} .
$$

Operators (A6) are always induced in a RG analysis of the DOS if one is after localization properties or in a RG analysis of the relevance of charge 2 vortices if one is after the fate of the Kosterlitz-Thouless transition in the 2D random phase $X Y$ model. Indeed, they are induced through repeated OPE's of the operator

$$
\mathcal{O}=e^{+2 \Phi_{1}}\left(\mathcal{B}_{+}+\mathcal{F}_{+}\right)+e^{-2 \Phi_{1}}\left(\mathcal{B}_{-}+\mathcal{F}_{-}\right)
$$

with itself. Hence, any RG analysis of the DOS or of the stability of the spin-wave phase in the 2D random phase $X Y$ model is controlled by the OPE's within the set $\left\{\mathcal{A}_{s ; N}\right\}$. These OPE's can be classified into three different processes which we call "fusion," "partial annihilation," and "complete annihilation" and are given by

$$
\begin{aligned}
& \mathcal{A}_{s ; N}(z, \bar{z}) \mathcal{A}_{s ; N^{\prime}}(w, \bar{w}) \\
& =|z-w|^{x_{N+N^{\prime}}-x_{N}-x_{N^{\prime}}}\left(\begin{array}{c}
N+N^{\prime} \\
N
\end{array}\right) \mathcal{A}_{s ; N+N^{\prime}}(w, \bar{w})+\cdots, \\
& 0<N, N^{\prime} \in \mathbb{N}, \quad s= \pm \text {, } \\
& \mathcal{A}_{+; N_{+}}(z, \bar{z}) \mathcal{A}_{-; N_{-}}(w, \bar{w})
\end{aligned}
$$

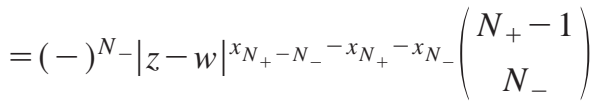

$$
\begin{aligned}
& \times \mathcal{A}_{+; N_{+}-N_{-}}(w, \bar{w})+\cdots, \\
& 0<N_{-}<N_{+} \in \mathbb{N} \text {, }
\end{aligned}
$$

$$
\begin{aligned}
& \mathcal{A}_{+; N_{+}}(z, \bar{z}) \mathcal{A}_{-; N_{-}}(w, \bar{w}) \\
& =(-)^{N}|z-w|^{2-2 x_{N}}\left\{+(N-1) \mathcal{O}_{A}(w, \bar{w})+N \mathcal{O}_{M}(w, \bar{w})\right. \\
& -2 N \partial \Phi_{1}(w)\left[-\bar{J}^{\prime}(\bar{w})+\bar{J}(\bar{w})\right] \\
& \left.+2 N \bar{\partial} \Phi_{1}(\bar{w})\left[-J^{\prime}(w)+J(w)\right]\right\}+\cdots, \\
& 0<N_{-}=N_{+}=N \in \mathbb{N} \text {, }
\end{aligned}
$$

respectively. These OPE's are the central result of this section. We have used the shorthand notation

$$
\begin{aligned}
\mathcal{O}_{A} & :=-\left(\psi_{-}^{*} \psi_{-}+\beta_{-}^{*} \beta_{-}\right)\left(\psi_{+}^{*} \psi_{+}+\beta_{+}^{*} \beta_{+}\right) \\
& =-\left(J^{\prime}-J\right)\left(\bar{J}^{\prime}-\bar{J}\right),
\end{aligned}
$$

for the random vector potential perturbation and

$$
\begin{aligned}
\mathcal{O}_{M} & :=-\left(\psi_{+}^{*} \psi_{-}+\beta_{+}^{*} \beta_{-}\right)\left(\psi_{-}^{*} \psi_{+}+\beta_{-}^{*} \beta_{+}\right) \\
& =+\left(J \bar{J}-J^{\prime} \bar{J}^{\prime}-G_{-} \bar{G}_{+}+G_{+} \bar{G}_{-}\right) \\
& =-\left(\mathcal{F}_{+} \mathcal{F}_{-}+\mathcal{B}_{+} \mathcal{B}_{-}+\mathcal{F}_{+} \mathcal{B}_{-}+\mathcal{F}_{-} \mathcal{B}_{+}\right)
\end{aligned}
$$

for the random mass perturbation. The gl(1|1) currents are defined by

$$
\begin{gathered}
J:=\psi_{-} \psi_{-}^{*}, \quad J^{\prime}:=\beta_{-} \beta_{-}^{*}, \\
G_{+}:=\beta_{-}^{*} \psi_{-}, \quad G_{-}:=\beta_{-} \psi_{-}^{*},
\end{gathered}
$$

with similar definitions in the antiholomorphic sectors for $\bar{J}, \bar{J}^{\prime}, \bar{G}_{+}, \bar{G}_{-}$. Operators $\mathcal{O}_{A}$ and $\mathcal{O}_{M}$ are induced by disorder averaging over a Gaussian-distributed real-valued random vector potential and random mass, respectively. We see that the process of complete annihilation induces a renormalization of $g_{A}$ through the presence of the terms $\partial \Phi_{1}\left(-J^{\prime}\right.$ $+J), \bar{\partial} \Phi_{1}\left(-\bar{J}^{\prime}+\bar{J}\right)$, and $\mathcal{O}_{A}$ on the right-hand side of Eq. (A9c). We can close the OPE's (A9) by inclusion of the OPE's $(s= \pm)$

$$
\begin{gathered}
\mathcal{O}_{M}(z, \bar{z}) \mathcal{A}_{s ; N}(w, \bar{w})=\frac{N(N-1)}{|z-w|^{2}} \mathcal{A}_{s ; N}(w, \bar{w})+\cdots, \\
\mathcal{O}_{M}(z, \bar{z}) \mathcal{O}_{M}(w, \bar{w})=\frac{-2}{|z-w|^{2}} \mathcal{O}_{A}(w, \bar{w})+\cdots
\end{gathered}
$$

It is consistent to neglect the process of complete annihilation (A9c) if one neglects OPE's (A13). This is the approximation that we make to calculate the DOS arbitrarily close to the band center.

\section{Case of two flavors}

We now return to the OPE's of the MDH model in Eq. (4.13) which we supplement by specifying the process of complete annihilation 


$$
\begin{aligned}
& \mathcal{A}_{s ; N}(z, \bar{z}) \mathcal{A}_{s ; N^{\prime}}(w, \bar{w}) \\
& =|z-w|^{x_{N+N^{\prime}}-x_{N}-x_{N^{\prime}}}\left(\begin{array}{c}
N+N^{\prime} \\
N
\end{array}\right) \mathcal{A}_{s ; N+N^{\prime}}(w, \bar{w})+\cdots, \\
& 0<N, N^{\prime} \in \mathbb{N}, s= \pm \text {, } \\
& \mathcal{A}_{+; N_{+}}(z, \bar{z}) \mathcal{A}_{-; N_{-}}(w, \bar{w})
\end{aligned}
$$

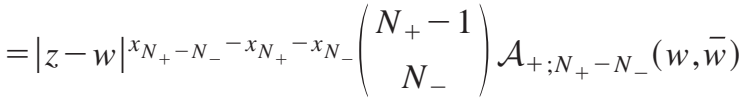

$$
\begin{aligned}
& +\cdots, \quad 0<N_{-}<N_{+} \in \mathbb{N}, \\
& \mathcal{A}_{+; N_{+}}(z, \bar{z}) \mathcal{A}_{-; N_{-}}(w, \bar{w}) \\
& =|z-w|^{2-2 x_{N}}\left\{-(N-1) \mathcal{O}_{A}(w, \bar{w})+N \mathcal{O}_{0}(w, \bar{w})\right. \\
& +2 N \partial \Phi_{2}(w) \sum_{a=1}^{2}\left[-\bar{J}_{a a}^{\prime}(\bar{w})+\bar{J}_{a a}(\bar{w})\right] \\
& \left.+2 N \bar{\partial} \Phi_{2}(\bar{w}) \sum_{a=1}^{2}\left[-J_{a a}^{\prime}(w)+J_{a a}(w)\right]\right\}+\cdots, \\
& 0<N_{+}=N_{-}=N \in \mathbb{N} \text {. }
\end{aligned}
$$

Here

$$
\begin{aligned}
\mathcal{O}_{A} & =-\sum_{a, b=1}^{2}\left(\psi_{a-}^{*} \psi_{a-}+\beta_{a-}^{*} \beta_{a-}\right)\left(\psi_{b+}^{*} \psi_{b+}+\beta_{b+}^{*} \beta_{b+}\right) \\
& =\sum_{a, b=1}^{2}\left[-J_{a a}^{\prime} \bar{J}_{b b}^{\prime}+J_{a a}^{\prime} \bar{J}_{b b}+J_{a a} \bar{J}_{b b}^{\prime}-J_{a a} \bar{J}_{b b}\right]
\end{aligned}
$$

is the random vector potential perturbation for the two-flavor case, and $\mathcal{O}_{0}$ is an operator generated by taking the OPE $\mathcal{A}_{+; 1} \times \mathcal{A}_{-; 1}$, whose explicit form is given by

$$
\begin{aligned}
\mathcal{O}_{0}= & +J_{11}^{\prime} \bar{J}_{22}^{\prime}+J_{12}^{\prime} \bar{J}_{21}^{\prime}+J_{21}^{\prime} \bar{J}_{12}^{\prime}+J_{22}^{\prime} \bar{J}_{11}^{\prime} \\
& -G_{11+} \bar{G}_{22+}-G_{12+} \bar{G}_{21+}+G_{21+} \bar{G}_{12+}+G_{22+} \bar{G}_{11+} \\
& -G_{11-} \bar{G}_{22-}-G_{12-} \bar{G}_{21-}+G_{21-} \bar{G}_{12-}+G_{22-} \bar{G}_{11-} \\
& -J_{11} \bar{J}_{22}+J_{12} \bar{J}_{21}+J_{21} \bar{J}_{12}-J_{22} \bar{J}_{11} .
\end{aligned}
$$

Observe that the multiplicative factor $(-)^{N}$ present in Eq. (A9) does not appear here. This is so because we find operators with negative scaling dimensions in the gauge-variant sector, i.e., through "Cooper-like" terms, when the random vector potential is imaginary. As was the case for a realvalued random vector potential, a renormalization of $g_{A}$ is encoded by the complete fusion process through the contributions $\partial \Phi_{2} \Sigma_{a}\left(-\bar{J}_{a a}^{\prime}+\bar{J}_{a a}\right), \bar{\partial} \Phi_{2} \Sigma_{a}\left(-J_{a a}^{\prime}+J_{a a}\right)$, and $\mathcal{O}_{A}$. For the MDH model, we neglect this effect in the same way as we neglect the renormalization of $g_{A}$ induced by the source term needed to compute the DOS. This approximation should be good if one is after the DOS asymptotically close to the band center but fails if one is interested in the DOS at large energy scales. For the HWK model, we neglect complete annihilation relative to the renormalization of $g_{A}$ induced by $g_{M}$.

\section{APPENDIX B: BOSONIZATION OF THE GHOST SYSTEM}

\section{Bosonization}

The purpose of this section is to review the steps of Ref. 27 by which the SUSY action

$$
\begin{aligned}
S_{0}= & \int \frac{d^{2} \boldsymbol{r}}{\pi}\left(\psi_{-}^{*} \bar{\partial} \psi_{-}+\psi_{+}^{*} \partial \psi_{+}\right) \\
& +\int \frac{d^{2} \boldsymbol{r}}{\pi}\left(\beta_{-}^{*} \bar{\partial} \beta_{-}+\beta_{+}^{*} \partial \beta_{+}\right),
\end{aligned}
$$

where $\psi^{*}, \psi$ are fermionic spinors and $\beta^{*}, \beta$ are bosonic spinors, can be "bosonized," i.e., represented by a pair of independent scalar fields $\phi$ and $\phi^{\prime}$ and a pair of independent fermionic fields $\chi^{*}$ and $\chi$. The fermionic sector in the SUSY action (B1) describes a CFT with central charge $c=+1$. The bosonic sector in the SUSY action (B1) describes a CFT with central charge $c=-1$. Taken together, both sectors describe a CFT with vanishing central charge. The OPE for the spinors are

$$
\begin{gathered}
\psi_{-}(z) \psi_{-}^{*}(0)=\psi_{-}^{*}(z) \psi_{-}(0)=\frac{1}{z}+\cdots, \\
\psi_{+}(\bar{z}) \psi_{+}^{*}(0)=\psi_{+}^{*}(\bar{z}) \psi_{+}(0)=\frac{1}{\bar{z}}+\cdots, \\
\beta_{-}(z) \beta_{-}^{*}(0)=-\beta_{-}^{*}(z) \beta_{-}(0)=\frac{1}{z}+\cdots, \\
\beta_{+}(\bar{z}) \beta_{+}^{*}(0)=-\beta_{+}^{*}(\bar{z}) \beta_{+}(0)=\frac{1}{\bar{z}}+\cdots
\end{gathered}
$$

After defining the dimension-1 currents

$$
\begin{gathered}
J:=\psi_{-} \psi_{-}^{*}, \quad J^{\prime}:=\beta_{-} \beta_{-}^{*}, \\
\bar{J}:=\psi_{+} \psi_{+}^{*}, \quad \bar{J}^{\prime}:=\beta_{+} \beta_{+}^{*}, \\
G_{+}:=\beta_{-}^{*} \psi_{-}, \quad G_{-}:=\beta_{-} \psi_{-}^{*}, \\
\bar{G}_{+}:=\beta_{+}^{*} \psi_{+}, \quad \bar{G}_{-}:=\beta_{+} \psi_{+}^{*},
\end{gathered}
$$

we see that they generate the $\operatorname{gl}(1 \mid 1)$ supercurrent algebra

$$
\begin{gathered}
J(z) J(w)=\frac{1}{(z-w)^{2}}+\cdots, \\
J^{\prime}(z) J^{\prime}(w)=\frac{-1}{(z-w)^{2}}+\cdots,
\end{gathered}
$$




$$
\begin{gathered}
J(z) G_{ \pm}(w)= \pm \frac{1}{z-w} G_{ \pm}(w)+\cdots, \\
J^{\prime}(z) G_{ \pm}(w)= \pm \frac{1}{z-w} G_{ \pm}(w)+\cdots, \\
G_{\mp}(z) G_{ \pm}(w)=\frac{ \pm 1}{(z-w)^{2}}+\frac{-1}{z-w}\left[J(w)-J^{\prime}(w)\right]+\cdots
\end{gathered}
$$

The idea behind bosonization is to represent the SUSY action (B1) in terms of a new set of fields so as to preserve the OPE's (B2) and (B4). For example, it has been known since the early 1970's that the currents for the fermionic $c$ $=1$ sector can be mimicked by a scalar field $\phi(z, \bar{z})$ through the identifications

$$
J \equiv+(i \partial \phi), \quad \bar{J} \equiv-(i \bar{\partial} \phi),
$$

which preserve the $J-J$ OPE in Eq. (B4) provided $\phi(z, \bar{z})$ obeys the OPE

$$
\phi(z, \bar{z}) \phi(0)=-\ln z \bar{z}+\cdots .
$$

In turn, fermionic spinors can be expressed by the Mandelstam formulas

$$
\begin{gathered}
\psi_{-} \equiv u^{-1} \mathfrak{a}^{-1 / 2} e^{+i \varphi_{-},} \\
\psi_{-}^{*} \equiv u^{+1} \mathfrak{a}^{-1 / 2} e^{-i \varphi_{-},} \\
\psi_{+} \equiv\left(u^{*}\right)^{-1} \mathfrak{a}^{-1 / 2} e^{-i \varphi_{+}}, \\
\psi_{+}^{*} \equiv\left(u^{*}\right)^{+1} \mathfrak{a}^{-1 / 2} e^{+i \varphi_{+},}
\end{gathered}
$$

whereby $\phi(z, \bar{z})$ is decomposed into a holomorphic and antiholomorphic part according to $\phi(z, \bar{z})=\varphi_{-}(z)+\varphi_{+}(\bar{z})$. Here, an arbitrary complex number $u$ has been introduced, as it is not fixed by the OPE's (B2).

Although the $\beta^{*}-\beta$ subsystem is already bosonic, it can be advantageous to trade these spinors for another scalar field $\phi^{\prime}(z, \bar{z})=\varphi_{-}^{\prime}(z)+\varphi_{+}^{\prime}(\bar{z}) \cdot{ }^{54}$ As in the fermionic case, identification

$$
J^{\prime} \equiv+(i \partial \phi), \quad \bar{J}^{\prime} \equiv-(i \bar{\partial} \phi),
$$

is compatible with the $J^{\prime}-J^{\prime}$ OPE in Eq. (B4) provided $\phi^{\prime}$ satisfies

$$
\phi^{\prime}(z, \bar{z}) \phi^{\prime}(0)=+\ln z \bar{z}+\cdots .
$$

Observe that this OPE differs from the one in the $\phi$ sector by a sign. Since the $\beta^{*}-\beta$ sector can be thought of as a CFT with central charge $c=-1$ whereas it is natural to assign the central charge $c=+1$ to the $\phi^{\prime}$ sector, a conformal sector with central charge $c=-2$ must be supplemented to the $\phi^{\prime}$ sector. The need for an additional conformal sector can also be seen when tentatively writing down Mandelstam formulas for the bosonic spinors $\bar{\beta}_{ \pm}, \beta_{ \pm}$of the form (B7) with the substitution of $\varphi_{ \pm}$by $\varphi_{ \pm}^{\prime}$ as the exponentials $\exp \left( \pm i \varphi_{ \pm}^{\prime}\right)$ obey Fermi statistics. The correct bosonic statistics in the $\beta^{*}-\beta$ sector is implemented after the introduction of two pairs of independent fermions $\eta_{ \pm}$and $\xi_{ \pm}$in the holomorphic and antiholomorphic sectors, respectively, through the bosonization rules

$$
\begin{gathered}
\beta_{-} \equiv v^{+1} \mathfrak{a}^{+1 / 2} e^{+i \varphi_{-}^{\prime}} \eta_{-}, \\
\beta_{-}^{*} \equiv v^{-1} \mathfrak{a}^{+1 / 2} e^{-i \varphi_{-}^{\prime}} \partial \xi_{-}, \\
\beta_{+} \equiv\left(v^{*}\right)^{+1} \mathfrak{a}^{+1 / 2} e^{-i \varphi_{+}^{\prime}} \eta_{+}, \\
\beta_{+}^{*} \equiv\left(v^{*}\right)^{-1} \mathfrak{a}^{+1 / 2} e^{+i \varphi_{+}^{\prime}} \bar{\partial} \xi_{+} .
\end{gathered}
$$

As before, the arbitrary complex number $v$ has been introduced, as it is not fixed by the OPE's (B2), whereas $\eta_{-}, \xi_{-}$, $\eta_{+}$, and $\xi_{+}$are anticommuting fields with the conformal weights $(1,0),(0,0),(0,1)$, and $(0,0)$, respectively. Their OPE's are thus given by

$$
\begin{aligned}
& \xi_{-}(z) \eta_{-}(0)=\eta_{-}(z) \xi_{-}(0)=\frac{1}{z}+\cdots, \\
& \xi_{+}(\bar{z}) \eta_{+}(0)=\eta_{+}(\bar{z}) \xi_{+}(0)=\frac{1}{\bar{z}}+\cdots .
\end{aligned}
$$

Finally, the action (B1) has the bosonized representation

$$
S_{0}=\int \frac{d^{2} \boldsymbol{r}}{2 \pi}\left(\partial \phi \bar{\partial} \phi-\partial \phi^{\prime} \bar{\partial} \phi^{\prime}\right)+\int \frac{d^{2} \boldsymbol{r}}{\pi}\left(\eta_{-} \bar{\partial} \xi_{-}+\eta_{+} \partial \xi_{+}\right) .
$$

Bosonization of the $\beta^{*}-\beta$ sector is not unique. ${ }^{55}$ Instead of the $\eta-\xi$ sector we could have chosen to introduce (symplectic) fermions through the action

$$
-\int \frac{d^{2} \boldsymbol{r}}{2 \pi}\left(\partial \chi^{*} \bar{\partial} \chi+\bar{\partial} \chi^{*} \partial \chi\right)
$$

the OPE's

$$
\chi^{*}(z, \bar{z}) \chi(0)=-\chi(z, \bar{z}) \chi^{*}(0)=-\ln z \bar{z}+\cdots
$$

and the identifications

$$
\begin{aligned}
& \eta_{-}(z) \equiv i \partial \chi^{*}(z), \quad \partial \xi_{-}(z) \equiv i \partial \chi(z), \\
& \eta_{+}(\bar{z}) \equiv i \bar{\partial} \chi^{*}(\bar{z}), \quad \bar{\partial} \xi_{+}(\bar{z}) \equiv i \bar{\partial} \chi(\bar{z}) .
\end{aligned}
$$

[The minus sign in Eq. (B13) is necessary to get the minus sign on the right-hand side of the OPE (B14) with the convention we are using for Grassmann integration.] With the Mandelstam formulas, fermionic currents are bosonized as

$$
\begin{gathered}
G_{-} \equiv(u v)\left[e^{+i\left(\varphi_{-}^{\prime}-\varphi_{-}\right)}\left(i \partial \chi^{*}\right)\right], \\
\bar{G}_{-} \equiv(u v)^{*}\left[e^{-i\left(\varphi_{+}^{\prime}-\varphi_{+}\right)}\left(i \bar{\partial} \chi^{*}\right)\right],
\end{gathered}
$$




$$
\begin{gathered}
G_{+} \equiv(u v)^{-1}\left[e^{-i\left(\varphi_{-}^{\prime}-\varphi_{-}\right)}(i \partial \chi)\right], \\
\bar{G}_{+} \equiv\left[(u v)^{-1}\right]^{*}\left[e^{+i\left(\varphi_{+}^{\prime}-\varphi_{+}\right)}(i \bar{\partial} \chi)\right] .
\end{gathered}
$$

This bosonization rule, together with Eqs. (B5) and (B8), is compatible with the $\mathrm{gl}(1 \mid 1)$ super current algebra OPE's (B4) for any value of $u, v \in \mathrm{C}$.

The ambiguity in the choice of the phase of $(u v)$ when bosonizing fermionic currents is, however, lifted in the presence of $\mathrm{gl}(1 \mid 1)$ current-current interactions by imposing boundary conditions on the bosonized fields that maintain SUSY. To see this, consider the action [recall Eq. (5.5b)]

$$
S=S_{0}-\frac{g_{A}}{2 \pi} \int \frac{d^{2} \boldsymbol{r}}{\pi} \mathcal{O}_{A}(\boldsymbol{r})-\frac{g_{M}}{2 \pi} \int \frac{d^{2} \boldsymbol{r}}{\pi} \mathcal{O}_{M}(\boldsymbol{r}),
$$

which describes action (B12) perturbed by the random vector potential $\mathcal{O}_{A}$ and the random mass $\mathcal{O}_{M}$ defined in Eqs. (A10) and (A11), respectively. Although $S$ is not conformally invariant it is still GL(1|1) symmetric. Consequently, the correlator $\left\langle\mathcal{O}_{M}(z, \bar{z}) \mathcal{O}_{M}(w, \bar{w})\right\rangle$, say, vanishes for any $g_{A}$ and $g_{M}$. Calculation of this correlation function can be done nonperturbatively as a function of $g_{A}$ and $g_{M}$ along the lines of Ref. 27. The condition that this correlator vanishes uniquely specifies the boundary condition obeyed by the scalar fields and, in turn, the phase of $(u v)$. In this paper we choose the boundary condition for which the bosonized fields vanish at infinity in the CFT defined by

$$
S_{0}=\int \frac{d^{2} \boldsymbol{r}}{2 \pi}\left(\partial \phi \bar{\partial} \phi-\partial \phi^{\prime} \bar{\partial} \phi^{\prime}\right)-\int \frac{d^{2} \boldsymbol{r}}{2 \pi}\left(\partial \chi^{*} \bar{\partial} \chi+\bar{\partial} \chi^{*} \partial \chi\right) .
$$

This amounts to the choice $u v /|u v|=-i$ for which the Mandelstam formulas become (with $|u v|=1, u=v$, say)

$$
\begin{aligned}
& \psi_{-} \equiv \mathfrak{a}^{-1 / 2} e^{-i \pi / 4+i \varphi_{-},} \\
& \psi_{-}^{*} \equiv \mathfrak{a}^{-1 / 2} e^{+i \pi / 4-i \varphi_{-}}, \\
& \psi_{+} \equiv \mathfrak{a}^{-1 / 2} e^{+i \pi / 4-i \varphi_{+}}, \\
& \psi_{+}^{*} \equiv \mathfrak{a}^{-1 / 2} e^{-i \pi / 4+i \varphi_{+}},
\end{aligned}
$$

and

$$
\begin{gathered}
\beta_{-} \equiv \mathfrak{a}^{+1 / 2} e^{+i \pi / 4+i \varphi_{-}^{\prime}}\left(i \partial \chi^{*}\right), \\
\beta_{-}^{*} \equiv \mathfrak{a}^{+1 / 2} e^{-i \pi / 4-i \varphi_{-}^{\prime}}(i \partial \chi), \\
\beta_{+} \equiv \mathfrak{a}^{+1 / 2} e^{-i \pi / 4-i \varphi_{+}^{\prime}\left(i \bar{\partial} \chi^{*}\right),} \\
\beta_{+}^{*} \equiv \mathfrak{a}^{+1 / 2} e^{+i \pi / 4+i \varphi_{+}^{\prime}(i \bar{\partial} \chi) .}
\end{gathered}
$$

\section{Scaling dimensions of composite operators made of bosonic spinors}

The $\chi$ sector plays an essential role as it ensures that the $\beta$ sector obeys a bosonic algebra. It also guarantees that the central charge of action (B18a) vanishes and gives composite operators from the $\beta$ sector their correct scaling dimensions. To illustrate this last point we calculate the scaling dimension of the composite operator $\left(\beta_{+}^{*} \beta_{-}\right)^{n}$. Taking repeated Wick contractions, one verifies that the scaling dimension of $\left(\beta_{+}^{*} \beta_{-}\right)^{n}$ is $n$. On the other hand, bosonization rules (B18) give $\left(\beta_{+}^{*} \beta_{-}\right)^{n}=\mathfrak{a}^{+n} i^{n} e^{+i n \phi^{\prime}}\left(i \partial \chi^{*}\right)^{n}(i \bar{\partial} \chi)^{n}$. Since $e^{+i n \phi^{\prime}}$ contributes the negative scaling dimension $-n^{2}$ in view of the OPE obeyed in the $\phi^{\prime}$ sector, $\left(i \partial \chi^{*}\right)^{n}(i \bar{\partial} \chi)^{n}$ must contribute the anomalous scaling dimension $n^{2}+n$. That this is indeed so follows from noting that $\left(i \partial \chi^{*}\right)^{n}$ is generated by repeated OPE of $\left(i \partial \chi^{*}\right)$ with itself,

$$
\begin{aligned}
\left(i \partial \chi^{*}\right)^{n}(z)= & \frac{1}{(n-1) !} \cdots \frac{1}{1 !} \frac{1}{0 !}: \partial^{n-1}\left(i \partial \chi^{*}\right) \\
& \times \partial^{n-2}\left(i \partial \chi^{*}\right) \cdots \partial^{1}\left(i \partial \chi^{*}\right) \partial^{0}\left(i \partial \chi^{*}\right):(z) .
\end{aligned}
$$

[The notation $:(\cdots)$ : refers to normal ordering.] Since the conformal weight of $\left(i \partial \chi^{*}\right)$ is $(1,0)$, the conformal weight of $\left(i \partial \chi^{*}\right)^{n}$ reads

$$
(n+(n-1)+\cdots+1,0)=\left(\frac{n(n+1)}{2}, 0\right) .
$$

Accounting for the contribution from the antiholomorphic part $(i \bar{\partial} \chi)^{n}$ yields the anomalous scaling dimension $n(n$ $+1)$ for $\left(i \partial \chi^{*}\right)^{n}(i \bar{\partial} \chi)^{n}$.
${ }^{1}$ J. T. Edwards and D. J. Thouless, J. Phys. C 4, 453 (1971).

${ }^{2}$ F. Wegner, Z. Phys. B: Condens. Matter 44, 9 (1981).

${ }^{3}$ C. Pryor and A. Zee, Phys. Rev. B 46, 3116 (1992).

${ }^{4}$ I. A. Gruzberg, P. J. Hirschfeld, and A. V. Shytov, Phys. Rev. Lett. 87, 239703 (2001).

${ }^{5}$ F. J. Dyson, Phys. Rev. 92, 1331 (1953).

${ }^{6}$ G. Theodorou and M. H. Cohen, Phys. Rev. B 13, 4597 (1976).

${ }^{7}$ T. P. Eggarter and R. Riedinger, Phys. Rev. B 18, 569 (1978).

${ }^{8}$ R. Gade, Nucl. Phys. B 398, 499 (1993); R. Gade and F. Wegner, ibid. 360, 213 (1991); F. J. Wegner, Phys. Rev. B 19, 783 (1979).

${ }^{9}$ A. W. W. Ludwig, M. P. A. Fisher, R. Shankar, and G. Grinstein, Phys. Rev. B 50, 7526 (1994).
${ }^{10}$ A. A. Nersesyan, A. M. Tsvelik, and F. Wenger, Phys. Rev. Lett. 72, 2628 (1994); Nucl. Phys. B 438, 561 (1995).

${ }^{11}$ T. Senthil and M. P. A. Fisher, Phys. Rev. B 61, 9690 (2000).

${ }^{12}$ M. Bocquet, D. Serban, and M. R. Zirnbauer, Nucl. Phys. B 578, 628 (2000).

${ }^{13}$ P. W. Brouwer, A. Furusaki, I. A. Gruzberg, and C. Mudry, Phys. Rev. Lett. 85, 1064 (2000).

${ }^{14}$ A. Altland and M. Zirnbauer, Phys. Rev. B 55, 1142 (1997).

${ }^{15}$ D. A. Ivanov, J. Math. Phys. 43, 126 (2002), and references therein.

${ }^{16}$ R. Bundschuh, C. Cassanello, D. Serban, and M. R. Zirnbauer, Nucl. Phys. B 532, 689 (1998); Phys. Rev. B 59, 4382 (1999). 
${ }^{17}$ A. Altland and R. Merkt, Nucl. Phys. B 607, 511 (2001).

${ }^{18}$ O. Motrunich, K. Damle, and D. A. Huse, Phys. Rev. B 63, 224204 (2001).

${ }^{19}$ P. W. Brouwer, C. Mudry, B. D. Simons, and A. Altland, Phys. Rev. Lett. 81, 862 (1998).

${ }^{20}$ P. W. Brouwer, C. Mudry, and A. Furusaki, Phys. Rev. Lett. 84, 2913 (2000); M. Titov, P. W. Brouwer, A. Furusaki, and C. Mudry, Phys. Rev. B 63, 235318 (2001).

${ }^{21}$ D. G. Shelton and A. M. Tsvelik, Phys. Rev. B 57, 14242 (1998).

${ }^{22}$ M. B. Hastings and S. L. Sondhi, Phys. Rev. B 64, 094204 (2001).

${ }^{23}$ A. Comtet, J. Desbois, and C. Monthus, Ann. Phys. (N.Y.) 239, 312 (1995).

${ }^{24}$ L. Balents and M. P. A. Fisher, Phys. Rev. B 56, 12970 (1997).

${ }^{25}$ H. Mathur, Phys. Rev. B 56, 15794 (1997).

${ }^{26}$ T. Fukui, Nucl. Phys. B 562, 477 (1999).

${ }^{27}$ S. Guruswamy, A. LeClair, and A. W. W. Ludwig, Nucl. Phys. B 583, 475 (2000).

${ }^{28}$ A. Altland and B. D. Simons, Nucl. Phys. B 562, 445 (1999).

${ }^{29}$ M. Fabrizio and C. Castellani, Nucl. Phys. B 583, 542 (2000).

${ }^{30}$ O. Motrunich, K. Damle, and D. A. Huse, Phys. Rev. B 65, 064206 (2002).

${ }^{31}$ C. Dasgupta and S. K. Ma, Phys. Rev. B 22, 1305 (1980).

${ }^{32}$ Y. Hatsugai, X.-G. Wen, and M. Kohmoto, Phys. Rev. B 56, 1061 (1997).

${ }^{33}$ Y. Morita and Y. Hatsugai, Phys. Rev. Lett. 79, 3728 (1997); Phys. Rev. B 58, 6680 (1998).

${ }^{34}$ S. Ryu and Y. Hatsugai, Phys. Rev. B 65, 033301 (2002).

${ }^{35}$ D. Carpentier and P. Le Doussal, Nucl. Phys. B 588, 565 (2000).

${ }^{36}$ C. de C. Chamon, C. Mudry, and X.-G. Wen, Phys. Rev. B 53, R7638 (1996).

${ }^{37}$ C. Mudry, C. Chamon, and X.-G. Wen, Nucl. Phys. B. 466, 383 (1996).

${ }^{38}$ C. C. Chamon, C. Mudry, and X.-G. Wen, Phys. Rev. Lett. 77, 4194 (1996).

${ }^{39}$ I. I. Kogan, C. Mudry, and A. M. Tsvelik, Phys. Rev. Lett. 77, 707 (1996).

${ }^{40}$ H. E. Castillo, C. de C. Chamon, E. Fradkin, P. M. Goldbart, and C. Mudry, Phys. Rev. B 56, 10668 (1997).

${ }^{41}$ C. Mudry and X.-G. Wen, Nucl. Phys. B 549, 613 (1999).

${ }^{42}$ B. Horovitz and P. Le Doussal, Phys. Rev. B 65, 125323 (2002).

${ }^{43}$ D. Bernard, Nucl. Phys. B 441, 471 (1995); D. Bernard, in Low-Dimensional Applications of Quantum Field Theory, edited by L. Baulieu, V. Kazakov, M. Picco, and P. Windey, Vol. 362 of NATO Advanced Study Institute, Series B: Physics (Plenum Press, New York, 1997); D. Bernard, hep-th/9509137 (unpublished).

${ }^{44}$ D. Carpentier and P. Le Doussal, Phys. Rev. E 63, 026110 (2001).

${ }^{45}$ B. Derrida and H. Spohn, J. Stat. Phys. 51, 817 (1988).

${ }^{46}$ E. Buffet, A. Patrick, and J. V. Pulé, J. Phys. A 26, 1823 (1993).

${ }^{47}$ M. P. A. Fisher and E. Fradkin, Nucl. Phys. B 251, 457 (1985); E. Fradkin, Phys. Rev. B 33, 3257 (1986); 33, 3263 (1986).

${ }^{48}$ If the "purely imaginary" gauge fields $i A_{0}$ and $i \mathbf{A}$ are analytically continued to the "purely real" gauge fields $A_{0}$ and $\mathbf{A}$, the operator $D_{\text {HWK }}$ in the upper right block of of Eq. (2.15a) becomes Hermitian. If so, $D_{\mathrm{HWK}}$ can be interpreted as the Hamiltonian for a single flavor of Dirac fermion subjected to random scalar, vector, and mass potentials studied in Refs. 9 and 43.

${ }^{49}$ The Hermitian energy perturbation in Eq. (5.7) of Ref. 27 differs from the non-Hermitian energy perturbation in Eq. (3.13c). Our choice has the advantage that the RG equations obeyed by the DOS has the same structure as had we chosen to work with the representation defined by Eq. (3.11).

${ }^{50}$ The dependences on $\Phi_{1}$ and $\Phi_{2}$ in the zero mode of $H_{\mathrm{MDH}}$ were inadvertently exchanged in Ref. 32.

${ }^{51}$ A. Kolmogorov, I. Petrovsky, and N. Piscounov, Moscow Univ. Math. Bull. (Engl. Transl.) 1, 1 (1937).

${ }^{52} \mathrm{M}$. Bramson, Convergence of Solutions of the Kolmogorov Equation to Traveling Waves, Memoirs of the American Mathematical Society, No. 285 (American Mathematical Society, Providence, RI, 1983).

${ }^{53}$ Neither the bosonization rules (4.14) and (4.15) nor the overall sign of the action (4.16) are chosen in a way consistent with Eq. (4.26) although this inconsistency has no bearing on the main results in Ref. 27 , as these were rederived independently in section 4.2 of Ref. 27 from a more general point of view. To fix this ambiguity, recall that bosonization rules follow from the $\mathrm{gl}(2 \mid 2)$ current algebra. This algebra is independent of the complexvalued parameter $u$ in Tables I and II. In order to preserve the SUSY of Eq. (5.5), bosonization rules must be supplemented by boundary conditions on the scalar fields $\phi_{a}$ and $\phi_{a}^{\prime}$ at infinity. The boundary conditions are uniquely fixed by the phase of the complex parameter $u$. The choice $u /|u|=-i$ imposes the boundary condition that the bosonized fields vanish at infinity. We are indebted to A. W. W. Ludwig for clarification of this point.

${ }^{54}$ D. Friedan, E. Martinec, and S. Shenker, Nucl. Phys. B 271, 93 (1986).

${ }^{55}$ M. R. Gaberdiel and H. G. Kausch, Phys. Lett. B 386, 131 (1996). See also H. G. Kausch, hep-th/9510149 (unpublished).

${ }^{56}$ L. Rozansky and H. Saleur, Nucl. Phys. B 376, 461 (1992).

${ }^{57}$ B. L. Altshuler, V. E. Kravtsov, and I. V. Lerner, JETP Lett. 43, 440 (1986); Sov. Phys. JETP 64, 1352 (1986); Phys. Lett. A 134, 488 (1989).

${ }^{58}$ O. N. Dorokhov, JETP Lett. 36, 318 (1982); P. A. Mello, P. Pereyra, and N. Kumar, Ann. Phys. (N.Y.) 181, 290 (1988).

${ }^{59}$ For a review see C. W. J. Beenakker, Rev. Mod. Phys. 69, 731 (1997).

${ }^{60}$ F. Wegner, Z. Phys. B: Condens. Matter 36, 209 (1980); in Localization and Metal Insulator Transitions, edited by $\mathrm{H}$. Fritzsche and D. Adler, Institute for Amorphous Studies Series (Plenum Press, New York, 1985).

${ }^{61}$ V. I. Fal'ko and K. B. Efetov, Europhys. Lett. 32, 627 (1995); Phys. Rev. B 52, 17413 (1996).

${ }^{62}$ J.-S. Caux, Phys. Rev. Lett. 81, 4196 (1998).

${ }^{63}$ A. Dohmen, P. Freche, and M. Janssen, Phys. Rev. Lett. 76, 4207 (1996).

${ }^{64}$ B. Huckestein and R. Klesse, Phys. Rev. B 55, R7303 (1997).

${ }^{65}$ A. D. Mirlin, Phys. Rep. 326, 259 (2000); A. D. Mirlin and F. Evers, Phys. Rev. B 62, 7920 (2000); F. Evers, A. Mildenberger, and A. D. Mirlin, ibid. 64, 241303(R) (2001).

${ }^{66}$ F. Wegner, Z. Phys. B: Condens. Matter 51, 279 (1983).

${ }^{67}$ M. R. Zirnbauer, hep-th/9905054 (unpublished).

${ }^{68}$ M. J. Bhaseen, I. I. Kogan, O. A. Soloviev, N. Taniguchi, and A. M. Tsvelik, Nucl. Phys. B 580, 688 (2000). 